\title{
Boundary conditions in supergravity on a manifold with boundary
}

\author{
Dmitry V. Belyaev* \\ Department of Physics and Astronomy, The Johns Hopkins University, \\ 3400 North Charles Street, Baltimore, MD 21218, USA \\ E-mail: dmitry.belyaev@desy.de
}

ABstRACT: We explain why it is necessary to use boundary conditions in the proof of supersymmetry of a supergravity action on a manifold with boundary. Working in both boundary ("downstairs") and orbifold ("upstairs") pictures, we present a bulk-plus-boundary/brane action for the five-dimensional (on-shell) supergravity which is supersymmetric with the use of fewer boundary conditions than were previously employed. The required Gibbons-Hawkinglike $Y$-term and many other aspects of the boundary/orbifold picture correspondence are discussed.

${ }^{*}$ Present address: DESY-T, Notkestrasse 85, 22603 Hamburg, Germany 


\section{Contents}

1. Introduction 3

2. Supersymmetry algebra 4

3. Boundary breaks supersymmetry 6

4. Boundary conditions needed for supersymmetry 7

4.1 Boundary condition on the gravitino 8

4.2 Boundary condition on the graviphoton 8

4.3 Are there other boundary conditions? 9

5. Bulk action 9

6. Boundary action for $\eta_{2}=0$

6.1 Variational principle 11

6.2 Supersymmetry without boundary conditions 13

7. Boundary action for $\eta_{2}=\alpha \eta_{1}$

7.1 Global $S U(2)$ rotation 14

7.2 Bulk-plus-boundary action

7.3 Supersymmetry of the action

7.4 Boundary conditions

7.5 $U(1)$ gauge invariance 17

8. Fate of $B_{M}$ terms 18

8.1 Old action 19

8.2 Supersymmetry with $B_{M}$ terms 19

8.3 New action 20

8.4 Extension to the $\alpha \neq 0$ case 21

9. From boundary to orbifold picture 22

9.1 Summary of the boundary picture discussion 22

9.2 Lifting to the orbifold

9.3 Boundary $Y$-term vs. Orbifold singular terms 24

9.4 Auxiliary boundary condition 25

9.5 Different $\varepsilon(z)$ for different fields 26

9.6 Another addition to the $Y$-term 27

9.7 Result: the orbifold action 28 
10. Supersymmetry in the orbifold picture 28

10.1 Bulk-plus-brane action 28

10.2 Supersymmetry variation of the bulk action 29

10.3 Supersymmetry variation of the brane action 30

10.4 Supersymmetry variation of the total action 31

10.5 Connection with earlier work 32

11. Fate of $B_{m}$ terms on the orbifold 33

$11.1 F_{m n}$ terms in the supersymmetry variation 33

$11.2 B_{m}$ terms in the supersymmetry variation

12. Gauge transformations on the orbifold 36

12.1 Breaking of the general coordinate invariance 36

12.2 Breaking of the $U(1)$ gauge invariance

12.3 Modified $U(1)$ gauge transformation

12.4 Modified supersymmetry transformations

13. Brane-localized matter 42

13.1 Preserving the bulk $U(1)$ gauge invariance

13.2 Modified Bianchi identity 44

14. Summary and Conclusions

A. Conventions 47

B. Gibbons-Hawking boundary term 49

C. Why we choose $n_{5}=-e_{5}^{\hat{5}}$

D. Our gauge 51

D.1 Metric tensor

D.2 Field strength 51

D.3 Spin connection 52

D.4 Extrinsic curvature

D.5 Advantages of the $e_{m}^{\hat{5}}=0$ gauge

E. Supersymmetry transformations

F. Bulk Lagrangian 


\section{Introduction}

In their ground-breaking paper [1], Horava and Witten discussed eleven-dimensional supergravity on a manifold with boundary, that arises as a low energy limit of the strongly coupled heterotic string theory. They explained that there are two possible descriptions of the same theory: the "downstairs" (boundary) picture and the "upstairs" (orbifold) picture. They made a comment, however, that working on the orbifold is technically more convenient, which was, perhaps, the reason why the orbifold picture became the de facto choice for many researchers working in this area.

One of such orbifold constructions is the now famous Randall-Sundrum scenario [2, 3] which is set up in a five-dimensional space-time with a negative cosmological constant. This scenario was supersymmetrized by different groups [4, 5, 6] with somewhat different approaches (see Ref. [7] for the proof of the equivalence of these approaches). The original orbifold construction was used for the supersymmetrization as well.

Over time people came to discover that the boundary ("downstairs" or "interval") picture is in many respects preferred over the orbifold picture. For example, in Ref. [8] it was demonstrated how the same physical content unambiguously encoded in the boundary picture can be obscured by various "twists" and "jumps" on the orbifold.

In this paper we provide more evidence for simplicity of the boundary picture.

We present a bulk-plus-boundary action with the five-dimensional gauged (on-shell) supergravity in the bulk. It is $N=2$ (locally) supersymmetric with a boundary condition on the supersymmetry parameter breaking a half of the bulk supersymmetries on the boundary. Supersymmetry of the action requires the use of a small subset of all the (natural) boundary conditions encoded in the action itself. Which boundary conditions are necessary is indicated by the supersymmetry algebra.

The boundary action is a sum of two terms. The first one is a Gibbons-Hawking-like term [9] (which we call "Y-term" to acknowledge the work of York [10, 11]). It allows the derivation of Neumann-like boundary conditions (which we call "natural" following Ref. [12]) from the standard variational principle in exactly the same way as equations of motion are derived. (The general variation of the action must vanish for arbitrary variations of the fields in the bulk and on the boundary.) The second term is (a half of) the brane action which one uses in the orbifold picture.

We present the transition from the boundary to the orbifold picture in detail. We find that the $Y$-term disappears in the transition. It is represented in the orbifold picture by brane-localized singularities of the bulk Lagrangian.

We explicitly check that the bulk-plus-brane action we obtain for the orbifold picture is supersymmetric upon using the same minimal set of boundary conditions as in the boundary picture. But we find that supersymmetry of the action requires introducing different $\varepsilon(z)$ (sign factor) assignments for odd fields compared to those previously assumed. Instead of the famous $\varepsilon(z)^{2} \delta(z)=1 / 3 \delta(z)$ we find that it is necessary to use another equally bizarre relation for a product of distributions: $\varepsilon(z)^{-2} \delta(z)=-\delta(z)$. 
The construction of Ref. [7] is obtained from the one presented here by explicitly using the natural boundary conditions (including those outside the minimal set) in the brane action and while performing the supersymmetry variation of the resulting bulk-plus-brane action. We find that in the approach of Ref. [7] the two alternative $\varepsilon(z)$ assignments cannot be distinguished.

We also find that in the orbifold picture all local transformations have to be modified by the explicit addition of brane-localized terms such that the resulting transformations are non-singular on the brane. (The hint for this modification appeared already in Refs. [4, [7], where the supersymmetry transformation of $\psi_{52}$ was modified.) Only after this modification the orbifold picture becomes equivalent to the boundary picture.

This paper is a companion to Ref. [13], where a detailed analysis of the Mirabelli and Peskin model [14] in both the boundary and the orbifold pictures is presented.

Our basic conventions are the same as in Ref. [7]. We summarize them in Appendix A. More details are included in Ref. [15].

\section{Supersymmetry algebra}

In this section we present the (on-shell) supersymmetry algebra of five-dimensional gauged supergravity. The (bulk) supergravity action and supersymmetry transformations are as in Ref. [7]. But in order to show an important feature of the local supersymmetry algebra, we need to include 3-Fermi terms in the supersymmetry transformation of the gravitino.

The complete form of the supersymmetry transformations is 1

$$
\begin{aligned}
\delta_{\mathcal{H}} e_{M}^{A}= & i \widetilde{\mathcal{H}}^{i} \Gamma^{A} \Psi_{M i} \\
\delta_{\mathcal{H}} B_{M}= & -i \frac{\sqrt{6}}{2} \widetilde{\mathcal{H}}^{i} \Psi_{M i} \\
\delta_{\mathcal{H}} \Psi_{M i}= & 2 D_{M}(\widehat{\omega}) \mathcal{H}_{i}+\frac{1}{2 \sqrt{6}}\left(\Gamma_{M}{ }^{N K}+4 \delta_{M}^{N} \Gamma^{K}\right) \widehat{F}_{N K} \mathcal{H}_{i} \\
& +\lambda Q_{i}{ }^{j}\left(\Gamma_{M}-\sqrt{6} B_{M}\right) \mathcal{H}_{j},
\end{aligned}
$$

where $Q_{i}{ }^{j}=i(\vec{q} \cdot \vec{\sigma})_{i}{ }^{j}$ and

$$
\begin{aligned}
& \widehat{F}_{M N}=F_{M N}+i \frac{\sqrt{6}}{4} \widetilde{\Psi}_{M}^{i} \Psi_{N i} \\
& \widehat{\omega}_{M A B}=\omega(e)_{M A B}-\frac{i}{4} e_{A}^{N} e_{B}^{K}\left(\widetilde{\Psi}_{N}^{i} \Gamma_{M} \Psi_{K i}+\widetilde{\Psi}_{M}^{i} \Gamma_{N} \Psi_{K i}-\widetilde{\Psi}_{M}^{i} \Gamma_{K} \Psi_{N i}\right)
\end{aligned}
$$

\footnotetext{
${ }^{1}$ The spinors $\Psi_{M i}$ and $\mathcal{H}_{i}$ are symplectic Majorana (see Appendix A). The index $i$ can be rotated by $U_{i}{ }^{j} \in S U(2): \Psi_{i}^{\prime}=U_{i}{ }^{j} \Psi_{j}$. The (global) $S U(2)$ is the automorphism symmetry group of the algebra when $\lambda \vec{q}=0$. The real vector $\vec{q}=\left(q_{1}, q_{2}, q_{3}\right)$ indicates which $U(1)$ subgroup of the $S U(2)$ has been gauged [16, 7 . One can set it to be a unit vector, $\vec{q}^{2}=1$.
} 
are supercovariant quantities (their supersymmetry variations contain no $\partial_{M} \mathcal{H}_{i}$ ). From these we can derive the (on-shell) supersymmetry algebra, ${ }^{2}$

$$
\left[\delta_{\text {susy }}(\Xi), \delta_{\text {susy }}(\mathcal{H})\right]=\delta_{\text {g.c. }}\left(v^{M}\right)+\delta_{\text {loc.L. }}\left(\omega^{A B}\right)+\delta_{U(1)}(u)+\delta_{\text {susy }}\left(\Upsilon_{i}\right)
$$

The commutator of two supersymmetry transformations with parameters $\mathcal{H}_{i}$ and $\Xi_{i}$ gives a general coordinate transformation (instead of a translation in the case of global supersymmetry) as well as other local transformations (in our case these are local Lorentz and $U(1)$ transformations). But what is special to local supersymmetry (see, e.g., Ref. [17]), the commutator also gives rise to another supersymmetry transformation! And the 3-Fermi terms in the supersymmetry transformations are essential to identify this feature.

A general coordinate transformation (with parameter $v^{M}$ ) has the same form on $e_{M}^{A}, B_{M}$ and $\Psi_{M i}$, since all of them carry the same world index $M$. Explicitly, on $B_{M}$ it is given by

$$
\delta_{v} B_{M}=v^{N} \partial_{N} B_{M}+B_{N} \partial_{M} v^{N} .
$$

The (local) Lorentz transformation (with parameter $\omega_{A B}=-\omega_{B A}$ ) is

$$
\delta_{\omega} e_{M}^{A}=e_{M}^{B} \omega_{B}^{A}, \quad \delta_{\omega} B_{M}=0, \quad \delta_{\omega} \Psi_{M i}=\frac{1}{4} \omega_{A B} \Gamma^{A B} \Psi_{M i}
$$

And the (local) $U(1)$ transformation (with parameter $u$ ) is as follows,

$$
\delta_{u} e_{M}^{A}=0, \quad \delta_{u} B_{M}=\partial_{M} u, \quad \delta_{u} \Psi_{M i}=u \frac{\sqrt{6}}{2} \lambda Q_{i}{ }^{j} \Psi_{M j}
$$

The parameters $v^{M}, \omega_{A B}, u$ and $\Upsilon_{i}$, which appear in the commutator (2.6), are given by

$$
\begin{aligned}
v^{M}= & 2 i \widetilde{\mathcal{H}}^{i} \Gamma^{M} \Xi_{i} \\
\omega^{A B}= & 2 i\left(\widetilde{\mathcal{H}}^{i} \Gamma^{K} \Xi_{i}\right) \widehat{\omega}_{K}^{A B}-2 i \lambda Q_{i}{ }^{j}\left(\widetilde{\mathcal{H}}^{i} \Gamma^{A B} \Xi_{j}\right) \\
& -\frac{i}{\sqrt{6}} \widetilde{\mathcal{H}}^{i} \Gamma^{A B}{ }_{N K} \Xi_{i} \widehat{F}^{N K}-\frac{4 i}{\sqrt{6}} \widetilde{\mathcal{H}}^{i} \Xi_{i} \widehat{F}^{A B} \\
u= & -2 i\left(\widetilde{\mathcal{H}}^{i} \Gamma^{K} \Xi_{i}\right) B_{K}-i \sqrt{6} \widetilde{\mathcal{H}}^{i} \Xi_{i} \\
\Upsilon_{i}= & -i\left(\widetilde{\mathcal{H}}^{j} \Gamma^{K} \Xi_{j}\right) \Psi_{K i} .
\end{aligned}
$$

The supersymmetry algebra tells us that in order for a (bulk-plus-boundary) action to be supersymmetric under the indicated supersymmetry transformations, it must also be invariant under the local transformations arising from the commutator (2.6). Namely, the general coordinate transformation, the local Lorentz transformation and the $U(1)$ gauge transformation. This allows one to find the boundary conditions necessary for supersymmetry of the action.

\footnotetext{
${ }^{2}$ The algebra closes exactly only on the bosonic fields $e_{M}^{A}$ and $B_{M}$. For the gravitino, $\Psi_{M i}$, additional non-closure terms appear, proportional to its equation of motion. For the off-shell supersymmetry algebra see Ref. [18].
} 


\section{Boundary breaks supersymmetry}

It is well-known that in the presence of a boundary half of the bulk supersymmetries are necessarily broken. The reason for this is the supersymmetry algebra, which generates a general coordinate transformation (a translation in the case of the global supersymmetry) in the direction normal to the boundary.

Indeed, let us consider an action on a manifold $\mathcal{M}$ with boundary $\partial \mathcal{M}$. Its variation under the general coordinate transformation gives rise to a boundary term,

$$
\delta_{v} S_{5}=\delta_{v} \int_{\mathcal{M}} \mathcal{L}_{5}=\int_{\mathcal{M}} D_{M}\left(v^{M} \mathcal{L}_{5}\right)=\int_{\partial \mathcal{M}} n_{M} v^{M} \mathcal{L}_{5}
$$

where $n^{M}$ is an outward pointing unit vector normal to the boundary. (The measures of integration, $d^{5} x e_{5}$ on $\mathcal{M}$ and $d^{4} x e_{4}^{\text {ind }}$ on $\partial \mathcal{M}$, are implicit. ) This is true for any action, provided $\mathcal{L}_{5}$ is a scalar under the general coordinate transformation. Thus, in the presence of a boundary, the action is not invariant under the general coordinate transformation unless

$$
n_{M} v^{M}=0 \quad \text { on } \partial \mathcal{M} \text {. }
$$

But the supersymmetry algebra shows that a commutator of two supersymmetry transformations generates the general coordinate transformation with

$$
v^{M}=2 i \widetilde{\mathcal{H}}^{i} \Gamma^{M} \Xi_{i}
$$

And thus a restriction on $v^{M}$ restricts the allowed supersymmetry transformations.

From now on we assume that the boundary is described by

$$
\partial \mathcal{M}: \quad x^{5}=\text { const . }
$$

The allowed general coordinate transformations,

$$
x^{M} \rightarrow x^{M}-v^{M}(x),
$$

with $n_{M} v^{M}=0$ on $\partial \mathcal{M}$, preserve this description, and thus our choice does not limit the general coordinate invariance any further. We also use a (finite) local Lorentz transformation to set $e_{m}^{\hat{5}}=0$ on $\partial \mathcal{M}$. In this gauge, which turns out to be very convenient for our discussion,

$$
e_{m}^{\hat{5}}=0, \quad e_{a}^{5}=0, \quad e_{5}^{a} \neq 0, \quad e_{\hat{5}}^{m} \neq 0
$$

and

$$
e_{m}^{a} e_{a}^{n}=\delta_{m}^{n}, \quad e_{a}^{m} e_{m}^{b}=\delta_{a}^{b}, \quad e_{5}^{5} e_{\hat{5}}^{5}=1, \quad e_{\hat{5}}^{m}=-e_{5}^{a} e_{a}^{m} e_{\hat{5}}^{5} .
$$

Note also that this is the gauge in which $e_{m}^{a}$ is a vierbein of the induced metric on the boundary (which is not true in general) and, therefore,

$$
e_{4}^{\text {ind }}=e_{4}=\operatorname{det} e_{m}^{a} .
$$


We also have $e_{5}=e_{4} e_{5}^{\hat{5}}$ and $n_{M}=\left(0,0,0,0, n_{5}\right)$ with (see Appendix D)

$$
n_{5}=-e_{5}^{\hat{5}} \text {. }
$$

After these simplifications and in the two-component spinor notation, Eqs. (3.2) and (3.3) give rise to the following condition,

$$
v^{5}=2 i \widetilde{\mathcal{H}}^{i} \Gamma^{5} \Xi_{i}=2 e_{\tilde{5}}^{5}\left(\eta_{2} \xi_{1}-\eta_{1} \xi_{2}\right)+\text { h.c. }=0 \quad \text { on } \partial \mathcal{M} .
$$

Here $\left(\eta_{1}, \eta_{2}\right)$ and $\left(\xi_{1}, \xi_{2}\right)$ are the constituents of $\mathcal{H}_{i}$ and $\Xi_{i}$, respectively. Clearly, its general solution is the following boundary condition on the supersymmetry parameter,

$$
\eta_{2}=\alpha \eta_{1} \quad \text { on } \partial \mathcal{M}
$$

where $\alpha$ is (for now) an arbitrary complex function of the boundary coordinates. (We will see, however, that we can find a supersymmetric bulk-plus-boundary action only for $\alpha=$ const.) This is exactly the boundary condition used in Ref. [7]. One linear combination of $\eta_{1}$ and $\eta_{2}$ gets fixed, while the orthogonal combination describes the unbroken supersymmetry transformation. We will first set $\alpha=0$ to simplify the discussion. ${ }^{3}$ Our boundary condition is then

$$
\eta_{2}=0 \quad \text { on } \partial \mathcal{M}
$$

The $N=2$ supersymmetry gets broken down to $N=1$ (described by $\eta_{1}$ ) due to the presence of the boundary.

Note that the breaking is on the boundary only. We will find a bulk-plus-boundary action which is invariant under the supersymmetry transformations with arbitrary $\eta_{1}$ and $\eta_{2}$ in the bulk of $\mathcal{M}$, restricted only by the boundary condition on $\partial \mathcal{M}$. (In contrast, in the case of the global supersymmetry, like in the Mirabelli and Peskin model, restricting constant $\eta_{1}$ and $\eta_{2}$ on $\partial \mathcal{M}$ is equivalent to restricting them everywhere, and thus the $N=2$ supersymmetry is really broken down to $N=1$.) However, in the corresponding effective four-dimensional theory one would be able to preserve only $N=1$ supersymmetry, because the second supersymmetry would be broken by the boundary conditions.

\section{Boundary conditions needed for supersymmetry}

Preserving the $N=1$ supersymmetry still requires some effort. In the Mirabelli and Peskin model we found that, in the boundary picture, a particular boundary action is required to preserve the $N=1$ supersymmetry. Off-shell, no boundary condition was necessary to establish supersymmetry of the bulk-plus-boundary action. On-shell, however, some boundary conditions (which are part of the auxiliary equations of motion) were necessary. In our (on-shell) supergravity case, we can find the boundary conditions that are important for supersymmetry directly from the supersymmetry algebra!

\footnotetext{
${ }^{3}$ It is sufficient to consider just the $\alpha=0$ case. Any other (constant) $\alpha$ is obtainable by a (global) $S U(2)$ rotation Ref. []]. See Section 0 for details.
} 


\subsection{Boundary condition on the gravitino}

We found that the commutator of two supersymmetry transformations generates another supersymmetry transformation with parameter

$$
\Upsilon_{i}=-i\left(\tilde{\mathcal{H}}^{j} \Gamma^{M} \Xi_{j}\right) \Psi_{M i}
$$

In our gauge $\left(e_{m}^{\hat{5}}=e_{a}^{5}=0\right)$ and with our boundary condition $\left(\eta_{2}=0\right.$ on $\left.\partial \mathcal{M}\right)$, we have

$$
\Upsilon_{i}=-e_{a}^{m}\left(i \eta_{1} \sigma^{a} \bar{\xi}_{1}+h . c .\right) \Psi_{m i}
$$

Writing $\Upsilon_{i}$ and $\Psi_{m i}$ in terms of their two-component constituents, $\left(\zeta_{1}, \zeta_{2}\right)$ and $\left(\psi_{m 1}, \psi_{m 2}\right)$, respectively, we obtain, in particular,

$$
\zeta_{2}=-e_{a}^{m}\left(i \eta_{1} \sigma^{a} \bar{\xi}_{1}+\text { h.c. }\right) \psi_{m 2}
$$

Thus, two allowed supersymmetry transformations (with $\eta_{2}=0$ and $\xi_{2}=0$ ) generate a forbidden supersymmetry transformation $\left(\zeta_{2} \neq 0\right)$, unless the boundary condition

$$
\psi_{m 2}=0 \quad \text { on } \partial \mathcal{M}
$$

is imposed. (If $\alpha \neq 0$, the boundary condition is $\psi_{m 2}=\alpha \psi_{m 1}$ on $\partial \mathcal{M}$.)

It is important to note, however, that the supersymmetry transformation in the commutator (2.6) arises from the 3-Fermi terms in the supersymmetry variation of the gravitino. Accordingly, we expect that the boundary condition (4.4) is needed to prove supersymmetry

of our action to all orders in fermions, but not just to quadratic order in fermions. We will show that it is indeed the case (provided an appropriate boundary action is included).

\subsection{Boundary condition on the graviphoton}

The commutator (2.6) also results in a $U(1)$ gauge transformation with parameter

$$
u=-2 i\left(\widetilde{\mathcal{H}}^{i} \Gamma^{K} \Xi_{i}\right) B_{K}-i \sqrt{6} \widetilde{\mathcal{H}}^{i} \Xi_{i}
$$

In our gauge $\left(e_{m}^{\hat{5}}=e_{a}^{5}=0\right)$ and with our boundary condition $\left(\eta_{2}=0\right.$ on $\left.\partial \mathcal{M}\right)$, we have

$$
u=-2 e_{a}^{m}\left(i \eta_{1} \sigma^{a} \bar{\xi}_{1}+\text { h.c. }\right) B_{m}
$$

This implies that there are two choices of boundary conditions compatible with supersymmetry of the (bulk-plus-boundary) action:

1. $B_{m} \neq 0$ on $\partial \mathcal{M}$; the action must be $U(1)$ gauge invariant; the boundary condition (if any) follows from maintaining the $U(1)$ invariance.

2. $B_{m}=0$ on $\partial \mathcal{M}$; the $U(1)$ gauge invariance is broken by this boundary condition and thus is not required of the action itself; the gauge invariance must not be broken in the bulk, however, since there the generated $u$ is non-zero. 
The first choice appears to be more attractive (gauge invariances lead to more controlled quantum field theories), but the second choice may also be required. In our setup this is the case when $\lambda q_{12} \neq 0$. The reason is that in this case the boundary condition $\eta_{2}=0$ on $\partial \mathcal{M}$ itself breaks the $U(1)$ gauge invariance! This happens because the $U(1)$ transformation acts on the supersymmetry parameter $\mathcal{H}_{i}$ in the same way as it does with $\Psi_{M i}$,

$$
\delta_{u} \mathcal{H}_{i}=u \frac{\sqrt{6}}{2} \lambda Q_{i}{ }^{j} \mathcal{H}_{j}
$$

For the two-component spinors this means

$$
\begin{aligned}
& \delta_{u} \eta_{1}=i \frac{\sqrt{6}}{2} \lambda u\left(q_{3} \eta_{1}-q_{12}^{*} \eta_{2}\right) \\
& \delta_{u} \eta_{2}=i \frac{\sqrt{6}}{2} \lambda u\left(-q_{12} \eta_{1}-q_{3} \eta_{2}\right) .
\end{aligned}
$$

We see that the boundary condition, $\eta_{2}=0$ on $\partial \mathcal{M}$, is not invariant under this transformation, unless $\lambda q_{12}=0$. (For $\alpha \neq 0$ the condition on $\vec{q}$ is accordingly modified.)

\subsection{Are there other boundary conditions?}

There are no other boundary conditions which are generated in the way described above. The Lorentz transformation does not generate a boundary condition since any supergravity Lagrangian is Lorentz invariant and, therefore, no boundary term is produced when varying the action.

This means that we should need (at most) two boundary conditions (on the gravitino and the graviphoton) to prove supersymmetry of the total bulk-plus-boundary action. In particular, we should be able to do this without using any boundary condition for the vielbein! And we will show explicitly that it is indeed so, provided an appropriate boundary action is found.

We would like to emphasize that despite the limited number of boundary conditions needed to prove supersymmetry of the total action, a dynamical setup should include a full set of boundary conditions to make the boundary value problem well defined. And such a set must itself be supersymmetric (though the use of equations of motion would be required to show the closure of the boundary conditions under supersymmetry if one works with the on-shell formulation of supergravity).

\section{Bulk action}

In this section we introduce the bulk supergravity action and consider its variation under the $U(1)$ gauge and the supersymmetry transformations.

Our bulk action is the standard gauged supergravity action in five dimensions [19]. We

will omit the 4-Fermi terms and will work to quadratic order in fermions. To this order, the 
action is

$$
\begin{aligned}
S_{5}=\int_{\mathcal{M}} d^{5} x e_{5} & -\frac{1}{2} R+\frac{i}{2} \widetilde{\Psi}_{M}^{i} \Gamma^{M N K} D_{N} \Psi_{K i} \\
& +6 \lambda^{2} \vec{q}^{2}+\frac{i}{2} \widetilde{\Psi}_{M}^{i} \Gamma^{M N K}\left[\frac{1}{2} \lambda Q_{i}{ }^{j}\left(\Gamma_{N}-\sqrt{6} B_{N}\right)\right] \Psi_{K i} \\
& -i \frac{\sqrt{6}}{16} F_{M N}\left(2 \widetilde{\Psi}^{M i} \Psi_{i}^{N}+\widetilde{\Psi}_{P}^{i} \Gamma^{M N P Q} \Psi_{Q i}\right) \\
& \left.-\frac{1}{4} F_{M N} F^{M N}-\frac{1}{6 \sqrt{6}} \epsilon^{M N P Q K} F_{M N} F_{P Q} B_{K}\right\} .
\end{aligned}
$$

Note that the action with $\lambda \neq 0$ can be obtained from the ungauged action (with $\lambda=0$ ) by modifying the covariant derivative on the gravitino,

$$
D_{M} \Psi_{N i} \quad \rightarrow \quad \widetilde{D}_{M} \Psi_{N i}=D_{M} \Psi_{N i}+\frac{1}{2} \lambda Q_{i}{ }^{j}\left(\Gamma_{M}-\sqrt{6} B_{M}\right) \Psi_{N j},
$$

and adding the cosmological constant term $6 \lambda^{2} \vec{q}^{2}$ to the Lagrangian. Similarly, the supersymmetry transformations are obtained by analogous modification of the covariant derivative on $\mathcal{H}_{i}$.

The modified derivative is covariant with respect to the $U(1)$ transformation,

$$
\delta_{u}\left(\widetilde{D}_{M} \Psi_{N i}\right)=u \frac{\sqrt{6}}{2} \lambda Q_{i}{ }^{j}\left(\widetilde{D}_{M} \Psi_{N j}\right)
$$

It is then clear that only the Chern-Simons term in the action is not invariant under the $U(1)$ transformation and, therefore,

$$
\begin{aligned}
\delta_{u} S_{5} & =\int_{\mathcal{M}} d^{5} x e_{5}\left\{-\frac{1}{6 \sqrt{6}} \epsilon^{M N P Q K} F_{M N} F_{P Q} \delta_{u} B_{K}\right\} \\
& =\int_{\mathcal{M}} d^{5} x e_{5} D_{K}\left[-u \frac{1}{6 \sqrt{6}} \epsilon^{M N P Q K} F_{M N} F_{P Q}\right] \\
& =\int_{\partial \mathcal{M}} d^{4} x e_{4}\left[-u \frac{1}{6 \sqrt{6}} n_{5} \epsilon^{5 M N P Q} F_{M N} F_{P Q}\right] .
\end{aligned}
$$

In our gauge $\left(e_{m}^{\hat{5}}=e_{a}^{5}=0, n_{5}=-e_{5}^{\hat{5}}\right)$ this becomes

$$
\delta_{u} S_{5}=\int_{\partial \mathcal{M}} d^{4} x e_{4}\left[u \frac{1}{6 \sqrt{6}} \epsilon^{m n p q} F_{m n} F_{p q}\right] .
$$

Therefore, the bulk action is $U(1)$ gauge invariant $\left(\delta_{u} S_{5}=0\right)$, provided the following (gauge invariant) boundary condition is imposed,

$$
F_{m n}=0 \quad \text { on } \partial \mathcal{M} .
$$

Another way to kill the boundary term is to require $u=0$ on $\partial \mathcal{M}$. The supersymmetry algebra then leads to a stronger (gauge non-invariant) boundary condition,

$$
B_{m}=0 \quad \text { on } \partial \mathcal{M} \text {. }
$$


Let us now consider the supersymmetry variation of the bulk action. One can show (see the details in Ref. [15]) that the action varies into a boundary term (the bulk part vanishes, since this action is known to be supersymmetric in the absence of boundary). Explicitly,

$$
\delta_{\mathcal{H}} S_{5}=\int_{\mathcal{M}} d^{5} x e_{5}\left(D_{M} \widetilde{K}^{M}\right)=\int_{\partial \mathcal{M}} d^{4} x e_{4}\left(n_{M} \widetilde{K}^{M}\right)
$$

where

$$
\begin{aligned}
\widetilde{K}^{M}= & -e^{M A} e^{N B} \delta_{\mathcal{H}} \omega_{N A B}-\frac{i}{2} \widetilde{\Psi}_{N}^{i} \Gamma^{N M K} \delta_{\mathcal{H}} \Psi_{K i} \\
& -F^{M N} \delta_{\mathcal{H}} B_{N}-\frac{4}{6 \sqrt{6}} \epsilon^{M N P Q K} F_{P Q} B_{K} \delta_{\mathcal{H}} B_{N} .
\end{aligned}
$$

In our gauge this simplifies to

$$
\begin{aligned}
\delta_{\mathcal{H}} S_{5}=\int_{\partial \mathcal{M}} d^{4} x e_{4}\{ & -e^{n a} \delta_{\mathcal{H}} \omega_{n a \hat{5}}+\left(\psi_{m 1} \sigma^{m n} \delta_{\mathcal{H}} \psi_{n 2}-\psi_{m 2} \sigma^{m n} \delta_{\mathcal{H}} \psi_{n 1}+\text { h.c. }\right) \\
& \left.-e_{5}^{\hat{5}} F^{n 5} \delta_{\mathcal{H}} B_{n}+\frac{4}{6 \sqrt{6}} \epsilon^{n p q k} F_{p q} B_{k} \delta_{\mathcal{H}} B_{n}\right\} .
\end{aligned}
$$

\section{Boundary action for $\eta_{2}=0$}

According to our discussion of boundary conditions necessary for supersymmetry of the action, if we

1) work to quadratic order in fermions, and

2) drop terms with $B_{M}$,

then there should exist a boundary action that makes the total bulk-plus-boundary action supersymmetric without the use of any boundary conditions (except the one on the supersymmetry parameter). In this section we present such an action.

\subsection{Variational principle}

In our analysis [13] of the Mirabelli and Peskin model [14], we found that the boundary action required for supersymmetry is, at the same time, the one which makes the variational principle well defined. Let us now turn this around. We will look for a boundary action which improves the variational principle and then see if it makes the total action supersymmetric.

If we consider the general variation of our action (which one would perform to find the equations of motion), we find

$$
\delta S_{5}=\int_{\mathcal{M}} d^{5} x e_{5}\left\{D_{M} K^{M}+(\mathrm{EOM}) \delta \Phi\right\}
$$


where $\Phi=\left\{e_{M}^{A}, B_{M}, \Psi_{M i}\right\}$ and

$$
\begin{aligned}
K^{M}= & -e^{M A} e^{N B} \delta \omega_{N A B}+\frac{i}{2} \widetilde{\Psi}_{N}^{i} \Gamma^{N M K} \delta \Psi_{K i} \\
& -F^{M N} \delta B_{N}-\frac{4}{6 \sqrt{6}} \epsilon^{M N P Q K} F_{P Q} B_{K} \delta B_{N} .
\end{aligned}
$$

Note that the only difference between $K^{M}$ and $\widetilde{K}^{M}$ is in the sign in front of the fermionic term. Accordingly, in our gauge $\left(e_{m}^{\hat{5}}=e_{a}^{5}=0, n_{5}=-e_{5}^{\hat{5}}\right)$ we obtain

$$
\begin{aligned}
\delta S_{5}=\int_{\partial \mathcal{M}} d^{4} x e_{4}\{ & -e^{n a} \delta \omega_{n a \hat{5}}-\left(\psi_{m 1} \sigma^{m n} \delta \psi_{n 2}-\psi_{m 2} \sigma^{m n} \delta \psi_{n 1}+\text { h.c. }\right) \\
& \left.-e_{5}^{5} F^{n 5} \delta B_{n}+\frac{4}{6 \sqrt{6}} \epsilon^{n p q k} F_{p q} B_{k} \delta B_{n}\right\}+(\mathrm{EOM}) .
\end{aligned}
$$

Let us now consider the following modified action,

$$
S_{5}^{\prime}=S_{5}+\int_{\partial \mathcal{M}} d^{4} x e_{4}\left[e^{m a} \omega_{m a \hat{5}}+\left(\psi_{m 1} \sigma^{m n} \psi_{n 2}+\text { h.c. }\right)\right] .
$$

In our gauge $\omega_{m a \hat{5}}$ is simply related to the extrinsic curvature (see Appendix $\mathbb{D}$ ),

$$
K_{m a}=\omega_{m a \hat{5}}, \quad K=e^{m a} \omega_{m a \hat{5}} .
$$

Therefore, the first term in the boundary action is just the standard Gibbons-Hawking term, which makes the variational problem for the total gravity action

$$
-\frac{1}{2} \int_{\mathcal{M}} R+\int_{\partial \mathcal{M}} K
$$

well defined. In our gauge this is especially easy to see. The general variation of the modified action,

$$
\begin{aligned}
\delta S_{5}^{\prime}=\int_{\partial \mathcal{M}} d^{4} x e_{4}\{ & \left(K_{m a}-K e_{m a}\right) \delta e^{m a}+\left(2 \psi_{m 2} \sigma^{m n} \delta \psi_{n 1}+\text { h.c. }\right) \\
& \left.-e_{5}^{5} F^{n 5} \delta B_{n}+\frac{4}{6 \sqrt{6}} \epsilon^{n p q k} F_{p q} B_{k} \delta B_{n}\right\}+(\mathrm{EOM})
\end{aligned}
$$

contains only the variation of the induced vierbein $e_{m}^{a}$ and not that of its normal derivative, which is exactly the purpose of the Gibbons-Hawking term. In addition, we see that the fermionic boundary term in the modified action (6.4) removes $\delta \psi_{n 2}$ from the boundary piece of the general variation. As a result, the boundary condition $\psi_{m 2}=0$ on $\partial \mathcal{M}$ (which is necessary to prove supersymmetry of the action to all orders in fermions) arises from requiring the general variation to vanish under arbitrary variation $\delta \psi_{m 1}$ on the boundary.

The expression for the supersymmetry variation of the modified action once again differs from the general variation (6.7) only in the fermionic piece (plus all the bulk terms are absent),

$$
\begin{aligned}
\delta_{\mathcal{H}} S_{5}^{\prime}=\int_{\partial \mathcal{M}} d^{4} x e_{4}\{ & \left(K_{m a}-K e_{m a}\right) \delta_{\mathcal{H}} e^{m a}+\left(2 \psi_{m 1} \sigma^{m n} \delta_{\mathcal{H}} \psi_{n 2}+\text { h.c. }\right) \\
& \left.-e_{5}^{\hat{5}} F^{n 5} \delta_{\mathcal{H}} B_{n}+\frac{4}{6 \sqrt{6}} \epsilon^{n p q k} F_{p q} B_{k} \delta_{\mathcal{H}} B_{n}\right\} .
\end{aligned}
$$




\subsection{Supersymmetry without boundary conditions}

Consider the following bulk-plus-boundary action,

$$
S=S_{5}+\int_{\partial \mathcal{M}} d^{4} x e_{4}\left[K+\left(\psi_{m 1} \sigma^{m n} \psi_{n 2}+h . c .\right)\right]+\int_{\partial \mathcal{M}} d^{4} x e_{4}\left(-3 \lambda_{1}\right),
$$

where we added an extra tension term to the boundary action. We now omit all $B_{M}$ terms and verify that the total bulk-plus-boundary action is supersymmetric without the use of any boundary conditions for gravitino or vierbein, but with the restriction on the supersymmetry parameter $\mathcal{H}_{i}{ }^{4}$

$$
\eta_{2}=0 \quad \text { on } \partial \mathcal{M}
$$

The supersymmetry variation gives

$$
\delta_{\mathcal{H}} S=\int_{\partial \mathcal{M}} d^{4} x e_{4}\left\{-\left(K^{m a}-K e^{m a}+3 \lambda_{1} e^{m a}\right) \delta_{\mathcal{H}} e_{m a}+\left(2 \psi_{m 1} \sigma^{m n} \delta_{\mathcal{H}} \psi_{n 2}+\text { h.c. }\right)\right\}
$$

where

$$
\begin{aligned}
\delta_{\mathcal{H}} e_{m a} & =-i \psi_{m 1} \sigma^{a} \bar{\eta}_{1}+\text { h.c. } \\
\delta_{\mathcal{H}} \psi_{m 2} & =-i K_{m a} \sigma^{a} \bar{\eta}_{1}+i \lambda q_{3} \sigma_{m} \bar{\eta}_{1} .
\end{aligned}
$$

With the help of the following identity,

$$
K_{n a} \sigma^{m n} \sigma^{a}=\frac{1}{2}\left(K_{m a}-K e_{m a}\right) \sigma^{a},
$$

we obtain

$$
\delta_{\mathcal{H}} S=\int_{\partial \mathcal{M}} d^{4} x e_{4}\left\{3\left(\lambda_{1}-\lambda q_{3}\right) i \psi_{m 1} \sigma^{m} \bar{\eta}_{1}+\text { h.c. }\right\}
$$

Therefore, the bulk-plus-boundary action $(6.9)$ is supersymmetric, provided $\lambda_{1}=\lambda q_{3}$. And no boundary condition for the gravitino or vierbein is needed to prove this.

But the boundary conditions do exist for this bulk-plus-boundary action. If we consider its general variation, we find

$$
\begin{aligned}
\delta S=\int_{\partial \mathcal{M}} d^{4} x e_{4}\{ & \left(K_{m a}-K e_{m a}+3 \lambda_{1} e_{m a}\right) \delta e^{m a} \\
& \left.+\left(2 \psi_{m 2} \sigma^{m n} \delta \psi_{n 1}+\text { h.c. }\right)\right\}+(\mathrm{EOM}) .
\end{aligned}
$$

\footnotetext{
${ }^{4} \mathrm{~A}$ similar bulk-plus-boundary action (for a spinning string in the superconformal gauge), supersymmetric with the use of only a boundary condition on the supersymmetry parameter, was obtained in 1982 in Ref. [20]; see their Eq. (5.7). (See also Eq. (5.17) in Ref. 21].) Analogous result for a spinning membrane, presented in a more geometrical setting, appeared in 1989 in Ref. 22]; see their Eq. (5.16).
} 
If we require this to vanish under arbitrary field variations, then in addition to the bulk equations of motion we obtain the following natural boundary conditions,

$$
K_{m a}=\lambda_{1} e_{m a}, \quad \psi_{m 2}=0 .
$$

If one allows the use of these boundary conditions to prove supersymmetry, then one can also claim that the following bulk-plus-boundary action,

$$
S_{5}+\int_{\partial \mathcal{M}} d^{4} x e_{4}\left(+\lambda_{1}\right)
$$

is supersymmetric (for $\lambda_{1}=\lambda q_{3}$ ). (This action is obtained from (6.9) by implementing the boundary condition in its boundary term.) This is the approach taken in Ref. 8$].^{5}$

Our bulk-plus-boundary action (6.9) has the advantage of giving a unique boundary action with which the invariance under supersymmetry is independent of the boundary conditions. At the same time, it generates an acceptable set of natural boundary conditions via the variational principle.

\section{Boundary action for $\eta_{2}=\alpha \eta_{1}$}

Here we show explicitly that the $\alpha=0$ case can be rotated into the $\alpha \neq 0$ case and that the rotated bulk-plus-boundary action is once again supersymmetric without the use of any boundary conditions.

\subsection{Global $S U(2)$ rotation}

We use the fact that the bulk action and the supersymmetry transformations are invariant under the following (global) rotations of the fermions $\left(\Psi_{M i}\right.$ and $\left.\mathcal{H}_{i}\right)$ and parameters $\vec{q}$,

$$
\Psi_{M i}^{\prime}=U_{i}^{j} \Psi_{M j}, \quad \mathcal{H}_{i}^{\prime}=U_{i}{ }^{j} \mathcal{H}_{j}, \quad Q^{\prime}=U Q U^{\dagger},
$$

where $Q=i(\vec{q} \cdot \vec{\sigma})$ and $U$ is a constant matrix of the $S U(2)$ group. We employ a particular rotation from this class,

$$
\eta_{1}^{\prime}=\frac{\eta_{1}-\alpha^{*} \eta_{2}}{\sqrt{1+\alpha \alpha^{*}}}, \quad \eta_{2}^{\prime}=\frac{\alpha \eta_{1}+\eta_{2}}{\sqrt{1+\alpha \alpha^{*}}}
$$

(similarly for $\left(\psi_{m 1}, \psi_{m 2}\right)$ and $\left.\left(\psi_{51}, \psi_{52}\right)\right)$, together with

$$
q_{12}^{\prime}=\frac{q_{12}-\alpha^{2} q_{12}^{*}-2 \alpha q_{3}}{1+\alpha \alpha^{*}}, \quad q_{3}^{\prime}=\frac{\alpha q_{12}^{*}+\alpha^{*} q_{12}+\left(1-\alpha \alpha^{*}\right) q_{3}}{1+\alpha \alpha^{*}} .
$$

The inverse rotation is obtained simply by changing the sign of $\alpha$. In particular,

$$
\eta_{2}=\frac{-\alpha \eta_{1}^{\prime}+\eta_{2}^{\prime}}{\sqrt{1+\alpha \alpha^{*}}}
$$

Therefore,

$$
\eta_{2}=0 \quad \Rightarrow \quad \eta_{2}^{\prime}=\alpha \eta_{1}^{\prime}
$$

\footnotetext{
${ }^{5}$ The statement in Ref. [8] (see the sentence after Eq. (2.12)) that in the downstairs picture no boundary action is required is, in fact, erroneous. We find here that the boundary tension term of Eq. (6.18) is needed. This does not, however, affect the rest of the analysis in Ref. [8].
} 


\subsection{Bulk-plus-boundary action}

Performing this rotation on the action (6.9) and omitting the primes on the new fields, we obtain

$$
S^{(\alpha)}=S_{5}+\int_{\partial \mathcal{M}} d^{4} x e_{4}\left[K+\left(\psi_{m 1} \sigma^{m n} \psi_{n 2}+h . c .\right)\right]+\int_{\partial \mathcal{M}} d^{4} x e_{4} \mathcal{L}_{B}^{(\alpha)}
$$

where ${ }^{6}$

$$
\mathcal{L}_{B}^{(\alpha)}=-3 \lambda_{1}+\left(\alpha_{11} \psi_{m 1} \sigma^{m n} \psi_{n 1}+2 \alpha_{12} \psi_{m 1} \sigma^{m n} \psi_{n 2}+\alpha_{22} \psi_{m 2} \sigma^{m n} \psi_{n 2}+\text { h.c. }\right),
$$

and the parameters are given by

$$
\alpha_{11}=\frac{-\alpha}{1+\alpha \alpha^{*}}, \quad \alpha_{12}=\frac{-\alpha \alpha^{*}}{1+\alpha \alpha^{*}}, \quad \alpha_{22}=\frac{\alpha^{*}}{1+\alpha \alpha^{*}} .
$$

We claim that this bulk-plus-boundary action is supersymmetric without the use of any boundary conditions for vierbein or gravitino (omitting the $B_{M}$ terms and working to second order in fermions), provided only that the supersymmetry parameter $\mathcal{H}_{i}$ is restricted by the condition

$$
\eta_{2}=\alpha \eta_{1} \quad \text { on } \partial \mathcal{M}
$$

\subsection{Supersymmetry of the action}

The supersymmetry variation gives

where

$$
\begin{aligned}
\delta_{\mathcal{H}} S^{(\alpha)}=\int_{\partial \mathcal{M}} d^{4} x e_{4}\{- & \left(K^{m a}-K e^{m a}+3 \lambda_{1} e^{m a}\right) \delta_{\mathcal{H}} e_{m a} \\
+2 & {\left[\left(\alpha_{11} \psi_{m 1}+\alpha_{12} \psi_{m 2}\right) \sigma^{m n} \delta_{\mathcal{H}} \psi_{n 1}\right.} \\
+ & \left.\left.\left(\alpha_{22} \psi_{m 2}+\left(\alpha_{12}+1\right) \psi_{m 1}\right) \sigma^{m n} \delta_{\mathcal{H}} \psi_{n 2}+\text { h.c. }\right]\right\},
\end{aligned}
$$

$$
\begin{aligned}
\delta e_{m}^{a} & =-i\left(\psi_{m 1} \sigma^{a} \bar{\eta}_{1}+\psi_{m 2} \sigma^{a} \bar{\eta}_{2}\right)+h . c . \\
\delta \psi_{m 1} & =2 \widehat{D}_{m} \eta_{1}+i K_{m a} \sigma^{a} \bar{\eta}_{2}+i \lambda \sigma_{m}\left(q_{3} \bar{\eta}_{2}+q_{12}^{*} \bar{\eta}_{1}\right) \\
\delta \psi_{m 2} & =2 \widehat{D}_{m} \eta_{2}-i K_{m a} \sigma^{a} \bar{\eta}_{1}+i \lambda \sigma_{m}\left(q_{3} \bar{\eta}_{1}-q_{12} \bar{\eta}_{2}\right) .
\end{aligned}
$$

Using $\eta_{2}=\alpha \eta_{1}$ (and assuming $\alpha=$ const), ${ }^{7}$ we can bring the variation to the following form,

$$
\begin{aligned}
\delta_{\mathcal{H}} S^{(\alpha)}=\int_{\partial \mathcal{M}} d^{4} x e_{4}\{ & 4 C_{1} \psi_{m 1} \sigma^{m n} \widehat{D}_{n} \eta_{1}+4 C_{2} \psi_{m 2} \sigma^{m n} \widehat{D}_{n} \eta_{1} \\
& +C_{3}\left(K_{a}^{m}-K e_{a}^{m}\right) i \psi_{m 1} \sigma^{a} \eta_{1}+C_{4}\left(K_{a}^{m}-K e_{a}^{m}\right) i \psi_{m 2} \sigma^{a} \eta_{1} \\
& \left.-3 C_{5} i \psi_{m 1} \sigma^{m} \bar{\eta}_{1}-3 C_{6} i \psi_{m 2} \sigma^{m} \bar{\eta}_{1}+\text { h.c. }\right\}
\end{aligned}
$$

${ }^{6}$ For a discussion of a boundary Lagrangian with general fermionic mass terms of the form $\alpha_{i j} \psi_{i} \psi_{j}$ see also Ref. [23].

${ }^{7}$ We were unable to find a bulk-plus-boundary action which would be supersymmetric for $\eta_{2}=\alpha \eta_{1}$ with $\alpha$ being a function of boundary coordinates. We can allow only $\alpha=$ const, despite the fact that the supersymmetry algebra does not explain this limitation. 
where the coefficients are

$$
\begin{aligned}
& C_{1}=\alpha_{11}+\alpha\left(\alpha_{12}+1\right) \\
& C_{2}=\alpha_{12}+\alpha \alpha_{22} \\
& C_{3}=\alpha_{11} \alpha^{*}-\alpha_{12} \\
& C_{4}=\alpha^{*}\left(\alpha_{12}+1\right)-\alpha_{22} \\
& C_{5}=-\lambda_{1}+\lambda A_{1} \\
& C_{6}=-\lambda_{1} \alpha^{*}+\lambda A_{2}
\end{aligned}
$$

with

$$
\begin{aligned}
& A_{1}=\alpha_{11}\left(q_{3} \alpha^{*}+q_{12}^{*}\right)+\left(\alpha_{12}+1\right)\left(q_{3}-q_{12} \alpha^{*}\right) \\
& A_{2}=\alpha_{12}\left(q_{3} \alpha^{*}+q_{12}^{*}\right)+\alpha_{22}\left(q_{3}-q_{12} \alpha^{*}\right) .
\end{aligned}
$$

Our bulk-plus-boundary action is supersymmetric without the use of boundary conditions for the fields if all the coefficients $C_{i}$ vanish. And indeed, using our expressions (7.8) for the parameters $\alpha_{i j}$, we find

$$
C_{1}=C_{2}=C_{3}=C_{4}=0 \text {. }
$$

We also find $A_{2}=\alpha^{*} A_{1}$ as well as

$$
A_{1}=-\frac{\alpha q_{12}^{*}+\alpha^{*} q_{12}+\left(\alpha \alpha^{*}-1\right) q_{3}}{1+\alpha \alpha^{*}}
$$

Therefore, the remaining conditions, $C_{5}=0$ and $C_{6}=0$, are satisfied provided we choose $\lambda_{1}$ in the boundary Lagrangian (7.7) to be

$$
\lambda_{1}=-\frac{\alpha q_{12}^{*}+\alpha^{*} q_{12}+\left(\alpha \alpha^{*}-1\right) q_{3}}{1+\alpha \alpha^{*}} \lambda .
$$

This is exactly the relation found in Ref. [7]. (Note also that it is just the rotated version of $\lambda_{1}=\lambda q_{3}$ for $\alpha=0$.) But, unlike Ref. [7] or [8], we did not have to use boundary conditions to prove supersymmetry of the total action.

\subsection{Boundary conditions}

The general variation of the bulk-plus-boundary action (7.6) gives

$$
\begin{aligned}
\delta S=\int_{\partial \mathcal{M}} d^{4} x e_{4}\{ & \left(K_{m a}-K e_{m a}+3 \lambda_{1} e_{m a}\right) \delta e^{m a} \\
+2[ & \left(\alpha_{11} \psi_{m 1}+\left(\alpha_{12}+1\right) \psi_{m 2}\right) \sigma^{m n} \delta \psi_{n 1} \\
& \left.\left.+\left(\alpha_{12} \psi_{m 1}+\alpha_{22} \psi_{m 2}\right) \sigma^{m n} \delta \psi_{n 2}+\text { h.c. }\right]\right\}+(\mathrm{EOM}) .
\end{aligned}
$$


One could worry that having both $\delta \psi_{m 1}$ and $\delta \psi_{m 2}$ in the boundary piece of the general variation could interfere with the application of the variational principle. And indeed, if we require the variation to vanish for arbitrary $\delta e^{m a}, \delta \psi_{m 1}$ and $\delta \psi_{m 2}$ on the boundary, we would obtain two fermionic boundary conditions,

$$
\psi_{m 2}=-\frac{\alpha_{11}}{\alpha_{12}+1} \psi_{m 1}, \quad \psi_{m 2}=-\frac{\alpha_{12}}{\alpha_{22}} \psi_{m 1}
$$

For general $\alpha_{i j}$ this would overdetermine the boundary value problem. However, for our special choice (7.8), the two boundary conditions reduce to one! This saves the variational principle.

Our bulk-plus-boundary action leads to the following natural boundary conditions,

$$
K_{m a}=\lambda_{1} e_{m a}, \quad \psi_{m 2}=\alpha \psi_{m 1}
$$

These, once again, coincide with the boundary conditions used in Refs. [7] and [8]. And if we plug these boundary conditions in our boundary Lagrangian (7.7), we find

$$
\mathcal{L}_{B}^{(\alpha)}=-3 \lambda_{1}-\left(\alpha \psi_{m 1} \sigma^{m n} \psi_{n 1}+\text { h.c. }\right)
$$

which is (a half of) the brane Lagrangian in Ref. [7].

\section{5 $U(1)$ gauge invariance}

We now investigate when the boundary action and/or boundary conditions are gauge invariant. The $U(1)$ gauge transformation on the fermions is

$$
\begin{aligned}
& \delta_{u} \eta_{1}=i w\left(q_{3} \eta_{1}-q_{12}^{*} \eta_{2}\right) \\
& \delta_{u} \eta_{2}=i w\left(-q_{3} \eta_{2}-q_{12} \eta_{1}\right)
\end{aligned}
$$

where $w \equiv i \frac{\sqrt{6}}{2} \lambda u \in \mathbb{R}$; the transformation on the $\psi_{m 1,2}$ is similar.

The boundary condition $\eta_{2}=\alpha \eta_{1}$ is gauge invariant if

$$
\delta_{u}\left(\eta_{2}-\alpha \eta_{1}\right)=-i w\left[\left(q_{12}+\alpha q_{3}\right)-\alpha\left(\alpha q_{12}^{*}-q_{3}\right)\right]=0 .
$$

This leads to the following quadratic equation for $\alpha$,

$$
q_{12}-\alpha^{2} q_{12}^{*}+2 \alpha q_{3}=0
$$

It is equivalent to two linear equations,

$$
\alpha q_{12}^{*}-q_{3}= \pm 1, \quad q_{12}+\alpha q_{3}= \pm \alpha
$$

where the signs \pm correspond to the two solutions of the quadratic equation [7]. (We assumed here the normalization condition $\vec{q}^{2}=1$ of Ref. [7].) 
The fermionic part of the boundary Lagrangian (including the $\psi_{m 1} \sigma^{m n} \psi_{n 2}$ term) is

$$
\mathcal{L}_{B F}=\frac{1}{1+\alpha \alpha^{*}}\left[-\alpha \psi_{1} \psi_{1}+\left(1-\alpha \alpha^{*}\right) \psi_{1} \psi_{2}+\alpha^{*} \psi_{2} \psi_{2}\right]
$$

where we used a shorthand notation $\psi_{i} \psi_{j} \equiv \psi_{m i} \sigma^{m n} \psi_{n j}$ and dropped the "+h.c.". (Note that $\mathcal{L}_{B F}$ vanishes when the boundary condition $\psi_{m 2}=\alpha \psi_{m 1}$ is used!) Its variation under the gauge transformation gives

$$
\delta_{u} \mathcal{L}_{B F}=\frac{i w}{1+\alpha \alpha^{*}}\left[W \psi_{1} \psi_{1}+2\left(\alpha q_{12}^{*}-\alpha^{*} q_{12}\right) \psi_{1} \psi_{2}+W^{*} \psi_{2} \psi_{2}\right]
$$

where

$$
W=\alpha\left(\alpha^{*} q_{12}-q_{3}\right)-\left(\alpha q_{3}+q_{12}\right)
$$

We see that the variation vanishes when $\alpha$ and $\vec{q}$ are related as in Eq. (7.28). We conclude, therefore, that the (full) boundary Lagrangian and the boundary condition $\eta_{2}=\alpha \eta_{1}$ are both gauge invariant when the equation $(7.28)$ is satisfied!

For future reference, we note that if we do not include the $\psi_{m 1} \sigma^{m n} \psi_{n 2}$ term, that is if we consider the variation of

$$
\mathcal{L}_{B F}^{(\alpha)}=\frac{1}{1+\alpha \alpha^{*}}\left[-\alpha \psi_{1} \psi_{1}-2 \alpha \alpha^{*} \psi_{1} \psi_{2}+\alpha^{*} \psi_{2} \psi_{2}\right]
$$

under the gauge transformation, we find

$$
\delta_{u} \mathcal{L}_{B F}^{(\alpha)}=\frac{i w}{1+\alpha \alpha^{*}}\left[\widetilde{W} \psi_{1} \psi_{1}+2\left(\alpha q_{12}^{*}-\alpha^{*} q_{12}\right) \psi_{1} \psi_{2}+\widetilde{W}^{*} \psi_{2} \psi_{2}\right]
$$

where

$$
\widetilde{W}=2 \alpha\left(\alpha^{*} q_{12}-q_{3}\right)
$$

Therefore, when the boundary condition $\eta_{2}=\alpha \eta_{1}$ is gauge invariant, i.e. the equation (7.28) is satisfied, the $\alpha$-dependent part of the boundary Lagrangian, $\mathcal{L}_{B}^{(\alpha)}$, is not gauge invariant! (Unless $\alpha=0$, in which case $\mathcal{L}_{B F}^{(\alpha)}=0$; or $\lambda=0$, in which case the $U(1)$ does not act on the fermions.)

\section{Fate of $B_{M}$ terms}

In this section we will keep all $B_{M}$ and $F_{M N}$ terms (as well as $e_{5}^{a}$ and $e_{\tilde{5}}^{m}$ ), and show that, with an appropriate addition to the boundary action, the bulk-plus-boundary action is supersymmetric provided we use the (gauge non-invariant) boundary condition $B_{m}=0$ on $\partial \mathcal{M}$. 


\subsection{Old action}

Consider the modified bulk action (6.4),

$$
S_{5}^{\prime}=S_{5}+\int_{\partial \mathcal{M}} d^{4} x e_{4}\left[K+\left(\psi_{m 1} \sigma^{m n} \psi_{n 2}+\text { h.c. }\right)\right] .
$$

Its general variation is (6.7),

$$
\begin{aligned}
\delta S_{5}^{\prime}=\int_{\partial \mathcal{M}} d^{4} x e_{4}\{ & -\left(K_{a}^{m}-K e_{a}^{m}\right) \delta e_{m}^{a}+\left(2 \psi_{m 2} \sigma^{m n} \delta \psi_{n 1}+\text { h.c. }\right) \\
& \left.-e_{5}^{5} F^{n 5} \delta B_{n}+\frac{4}{6 \sqrt{6}} \epsilon^{n p q k} F_{p q} B_{k} \delta B_{n}\right\}+(\mathrm{EOM}),
\end{aligned}
$$

and its supersymmetry variation is given by (6.8),

$$
\begin{aligned}
\delta_{\mathcal{H}} S_{5}^{\prime}=\int_{\partial \mathcal{M}} d^{4} x e_{4}\{ & -\left(K_{a}^{m}-K e_{a}^{m}\right) \delta_{\mathcal{H}} e_{m}^{a}+\left(2 \psi_{m 1} \sigma^{m n} \delta_{\mathcal{H}} \psi_{n 2}+\text { h.c. }\right) \\
& \left.-e_{5}^{5} F^{n 5} \delta_{\mathcal{H}} B_{n}+\frac{4}{6 \sqrt{6}} \epsilon^{n p q k} F_{p q} B_{k} \delta_{\mathcal{H}} B_{n}\right\} .
\end{aligned}
$$

This is true for any $\mathcal{H}_{i}$.

\subsection{Supersymmetry with $B_{M}$ terms}

Let us first discuss the case $\alpha=0$, so that the boundary condition on the supersymmetry parameter $\mathcal{H}_{i}$ is

$$
\eta_{2}=0 \quad \text { on } \partial \mathcal{M}
$$

The supersymmetry transformations then are

$$
\begin{aligned}
\delta_{\mathcal{H}} e_{m}^{a}= & -i \psi_{m 1} \sigma^{a} \bar{\eta}_{1}+\text { h.c. } \\
\delta_{\mathcal{H}} B_{m}= & i \frac{\sqrt{6}}{2} \psi_{m 2} \eta_{1}+\text { h.c. } \\
\delta_{\mathcal{H}} \psi_{m 2}= & -i K_{m a} \bar{\eta}_{1}+i \lambda q_{3} \sigma_{m} \bar{\eta}_{1} \\
& +i \sqrt{6} \lambda q_{12} \eta_{1} B_{m}-\frac{1}{2 \sqrt{6}}\left(i \epsilon_{m}{ }^{n k l} \sigma_{l}+4 \delta_{m}^{n} \sigma^{k}\right) \bar{\eta}_{1} F_{n k} .
\end{aligned}
$$

Therefore,

$$
\begin{aligned}
\delta_{\mathcal{H}} S_{5}^{\prime}= & \int_{\partial \mathcal{M}} d^{4} x e_{4}\left\{-3 i \lambda q_{3}\left(\psi_{m 1} \sigma^{m} \bar{\eta}_{1}\right)\right. \\
& +2 i \sqrt{6} \lambda q_{12}\left(\psi_{m 1} \sigma^{m n} \eta_{1}\right) B_{n}-\frac{3 i}{2 \sqrt{6}}\left(\psi_{m 1} \sigma_{n} \bar{\eta}_{1}\right) \epsilon^{m p q n} F_{p q} \\
& \left.-i \frac{\sqrt{6}}{2}\left(\psi_{n 2} \eta_{1}\right) e_{5}^{\hat{5}} F^{n 5}+\frac{i}{3}\left(\psi_{n 2} \eta_{1}\right) \epsilon^{n p q k} F_{p q} B_{k}+\text { h.c. }\right\}
\end{aligned}
$$


The term with $\lambda q_{3}$ can be compensated by a boundary tension term (see Eq. (6.9)). Most of the other terms can be killed by application of the boundary condition for $B_{m}$,

$$
F_{m n}=0 \quad \text { on } \partial \mathcal{M}
$$

if $\lambda q_{12}=0$ and the gauge invariance is preserved on the boundary; or

$$
B_{m}=0 \quad \text { on } \partial \mathcal{M}
$$

if $\lambda q_{12} \neq 0$ and the gauge invariance on the boundary is broken by the boundary condition on the supersymmetry parameter. However, we are still left with

$$
\delta_{\mathcal{H}} S_{5}^{\prime}=\int_{\partial \mathcal{M}} d^{4} x e_{4}\left\{-i \frac{\sqrt{6}}{2}\left(\psi_{m 2} \eta_{1}\right) e_{5}^{\hat{5}} F^{n 5}\right\}
$$

This remaining term can, in principle, be canceled by the boundary condition on the gravitino,

$$
\psi_{m 2}=0 \quad \text { on } \partial \mathcal{M}
$$

However, our analysis of the supersymmetry algebra indicates that we should not need this boundary condition for supersymmetry to quadratic order in fermions. Therefore, there should exist a boundary action which lets us avoid using this boundary condition.

Another reason in favor of modifying our action is that, at the moment, the $B_{m}$ boundary condition we need $\left(B_{m}=0\right.$ or $F_{m n}=0$ on $\left.\partial \mathcal{M}\right)$ is not the same as the natural boundary condition arising from the bulk-plus-boundary action,

$$
e_{5}^{\hat{5}} F^{n 5}-\frac{4}{6 \sqrt{6}} \epsilon^{n p q k} F_{p q} B_{k}=0 \quad \text { on } \partial \mathcal{M}
$$

\subsection{New action}

In order to

1) avoid using the $\psi_{m 2}=0$ boundary condition in proving supersymmetry (to quadratic order in fermions), and

2) have the boundary condition for $B_{m}\left(B_{m}=0\right.$ or $F_{m n}=0$ on $\left.\partial \mathcal{M}\right)$ appear as a natural boundary condition,

we need to add an appropriate boundary action. Such a boundary action exists, but it turns out that it itself breaks gauge invariance! That is, for agreement with the supersymmetry algebra we have to break the gauge invariance (only on the boundary) by hand. However, we will find that the same boundary action is also needed for the correct transition to the orbifold picture. 
The required boundary action is easy to find. Indeed, consider the following bulk-plusboundary action,

$$
S_{5}^{\prime \prime}=S_{5}^{\prime}+\int_{\partial \mathcal{M}} d^{4} x e_{4}\left\{e_{5}^{\hat{5}} F^{n 5} B_{n}\right\}
$$

Its general variation is,

$$
\begin{aligned}
\delta S_{5}^{\prime \prime}=\int_{\partial \mathcal{M}} d^{4} x e_{4}\{ & -\left[K_{a}^{m}-K e_{a}^{m}-\left(B_{n} C^{n}\right) e_{a}^{m}\right] \delta e_{m}^{a}+\left(2 \psi_{m 2} \sigma^{m n} \delta \psi_{n 1}+\text { h.c. }\right) \\
& \left.+B_{m} \delta C^{m}+\frac{4}{6 \sqrt{6}} \epsilon^{n p q k} F_{p q} B_{k} \delta B_{n}\right\}+(\mathrm{EOM})
\end{aligned}
$$

where we defined

$$
\begin{aligned}
C^{m} \equiv e_{5}^{\hat{5}} F^{m 5} & =e_{5}^{\hat{5}}\left[g^{m n} g^{k 5} F_{n k}+\left(g^{m n} g^{55}-g^{m 5} g^{n 5}\right) F_{n 5}\right] \\
& =\gamma^{m n} F_{n \hat{5}} \equiv e_{a}^{m} e^{n a}\left(e_{\hat{5}}^{5} F_{n 5}+e_{\hat{5}}^{k} F_{n k}\right)
\end{aligned}
$$

The natural boundary conditions corresponding to independent variations of $B_{m}$ and $C^{m}$ (they are independent since $C^{m}$ involves $\partial_{5} B_{n}$ whose value on $\partial \mathcal{M}$ is independent of the value of $B_{n}$ ) coincide on the following boundary condition,

$$
B_{m}=0 \quad \text { on } \partial \mathcal{M}
$$

This is exactly the (gauge non-invariant) boundary condition dictated by the supersymmetry algebra. (Note that the $B_{m}$ and $C^{m}$ fields are analogous to, respectively, the $\Phi$ and $D=$ $X_{3}-\partial_{5} \Phi$ fields of the Mirabelli and Peskin model [14]. There, in order to derive the boundary condition for $\Phi$, we need a boundary term $\Phi D$ [13].)

The result of the supersymmetry variation, compared to Eq. (8.11), is now

$$
\delta_{\mathcal{H}} S_{5}^{\prime \prime}=\int_{\partial \mathcal{M}} d^{4} x e_{4}\left\{B_{n} \delta_{\mathcal{H}} C^{n}+\left(B_{n} C^{n}\right) e_{a}^{m} \delta_{\mathcal{H}} e_{m}^{a}\right\}
$$

And, therefore, the bulk-plus-boundary action (8.14) is supersymmetric upon using only the $B_{m}=0$ boundary condition, but not the $\psi_{m 2}=0$ one.

\subsection{Extension to the $\alpha \neq 0$ case}

The generalization to the $\alpha \neq 0$ case is straightforward. Since $B_{M}$ is not rotated under the $S U(2)$, we do not get new boundary terms for the $\alpha \neq 0$ case. The boundary condition remains $B_{m}=0$ on $\partial \mathcal{M}$. The only terms we should consider, therefore, are those containing $F_{m 5}$.

The variation (7.10) can also be written as follows,

$$
\begin{aligned}
& \delta_{\mathcal{H}} S^{(\alpha)}=\int_{\partial \mathcal{M}} d^{4} x e_{4}\{-\left(K^{m a}-K e^{m a}+3 \lambda_{1} e^{m a}\right) \delta_{\mathcal{H}} e_{m a} \\
&\left.+2\left[\frac{\psi_{m 1}+\alpha^{*} \psi_{m 2}}{1+\alpha \alpha^{*}} \sigma^{m n}\left(\delta_{\mathcal{H}} \psi_{n 2}-\alpha \delta_{\mathcal{H}} \psi_{n 1}\right)+\text { h.c. }\right]\right\} .
\end{aligned}
$$


The terms with $F_{m 5}$ appear only in the variation of the gravitino,

$$
\begin{aligned}
& \delta_{\mathcal{H}} \psi_{m 1}=\frac{-4 i}{2 \sqrt{6}}\left(\sigma_{m}{ }^{n}+\delta_{m}^{n}\right) \eta_{1}\left(e_{\tilde{5}}^{5} F_{n 5}\right)+\ldots \\
& \delta_{\mathcal{H}} \psi_{m 2}=\frac{-4 i}{2 \sqrt{6}}\left(\sigma_{m}{ }^{n}+\delta_{m}^{n}\right) \eta_{2}\left(e_{\tilde{5}}^{5} F_{n 5}\right)+\ldots
\end{aligned}
$$

where dots represent the terms already considered. But since $\eta_{2}=\alpha \eta_{1}$ on $\partial \mathcal{M}$, the $F_{m 5}$ terms cancel in the combination $\delta_{\mathcal{H}} \psi_{n 2}-\alpha \delta_{\mathcal{H}} \psi_{n 1}$ ! (Note that in the orbifold picture the cancellation of the $F_{m 5}$ terms is less straightforward, as we will see.) This completes our explicit check of the fact that the rotated action,

$$
S^{(\alpha)}+\int_{\partial \mathcal{M}} d^{4} x e_{4}\left\{e_{5}^{\hat{5}} F^{n 5} B_{n}\right\}
$$

is supersymmetric (using only the $\eta_{2}=\alpha \eta_{1}$ and $B_{m}=0$ boundary conditions).

\section{From boundary to orbifold picture}

In this section we will show that that the generalized Gibbons-Hawking term [9] (which we call " $Y$-term" to honor the work of York [10, 11]) matches onto the brane-localized singularities of the bulk Lagrangian in the orbifold picture. This explains why the $Y$-term appears only in the bulk-plus-boundary action (in the boundary picture), but not in the bulk-plus-brane action (in the orbifold picture).

\subsection{Summary of the boundary picture discussion}

Our total bulk-plus-boundary action is

$$
S=\int_{\mathcal{M}} d^{5} x e_{5} \mathcal{L}_{5}+\int_{\partial \mathcal{M}} d^{4} x e_{4} Y+\int_{\partial \mathcal{M}} d^{4} x e_{4} \mathcal{L}_{B}^{(\alpha)}
$$

where the $\alpha$-independent boundary term $Y$ is

$$
Y=K+\left(\psi_{m 1} \sigma^{m n} \psi_{n 2}+\text { h.c. }\right)+e_{5}^{\hat{5}} F^{n 5} B_{n}
$$

and $\mathcal{L}_{B}^{(\alpha)}$ is given by Eq. (7.7) together with Eqs. (7.8) and (7.20). We showed that the action is supersymmetric for $\eta_{2}=\alpha \eta_{1}$ on $\partial \mathcal{M}$ provided we use just one more boundary condition: $B_{m}=0$ on $\partial \mathcal{M}$.

The $Y$-term is a generalization of the Gibbons-Hawking boundary term for our bulk action. It allows us to derive (natural) boundary conditions by requiring that the general variation of the action vanish for arbitrary field variations in the bulk and on the boundary. The boundary conditions thus obtained are

$$
B_{m}=0, \quad K_{m a}=\lambda_{1} e_{m a}, \quad \psi_{m 2}=\alpha \psi_{m 1} \quad \text { on } \partial \mathcal{M} .
$$

They are consistent with supersymmetry, as was shown in Ref. [7]. Their supersymmetry variations also produce other (secondary) boundary conditions. In on-shell formulation, one can at most expect that the full system of boundary conditions closes under supersymmetry only up to equations of motion [13]. (See also Ref. [24].) 


\subsection{Lifting to the orbifold}

Let us now lift our results to the $\mathbb{R} / \mathbb{Z}_{2}$ orbifold. The orbifold can be constructed from two copies of our space-time manifold with boundary,

$$
\mathcal{M}_{-}=\mathbb{R}^{1,3} \times(-\infty, 0] \text { and } \mathcal{M}_{+}=\mathbb{R}^{1,3} \times[0,+\infty) .
$$

(We use the notation valid for the flat case [13], but keep in mind that we are actually on a general curved manifold.) Since the boundaries of $\mathcal{M}_{+}$and $\mathcal{M}_{-}$coincide, we denote $\Sigma=\partial \mathcal{M}_{+}=\partial \mathcal{M}_{-}$and call this hypersurface a "brane".

We use a $\mathbb{Z}_{2}$ symmetry of the bulk action to impose the following parity assignments [7] ("even/odd" means $\phi(-z)= \pm \phi(+z)$ ),

$$
\begin{aligned}
& \text { even : } e_{m}^{a} e_{5}^{\hat{5}} B_{5} \quad \psi_{m 1} \psi_{52} \eta_{1} q_{12} \lambda \\
& \text { odd : } e_{m}^{\hat{5}} e_{5}^{a} B_{m} \psi_{m 2} \psi_{51} \eta_{2} q_{3} \text {. }
\end{aligned}
$$

It follows that $K=e^{m a} \omega_{m a \hat{5}}$ is odd and $F^{m 5}$ is even. Therefore, the $Y$-term is odd.

Since we used $n_{5}=-e_{5}^{5}$, our bulk-plus-boundary action is appropriate for $\mathcal{M}_{+}$. For $\mathcal{M}_{-}$, we should use $n_{5}=+e_{5}^{\hat{5}}$ (see Appendix Q) and accordingly change the sign of the $Y$-term. The boundary conditions, which our actions for $\mathcal{M}_{+}$and $\mathcal{M}_{-}$should reproduce, are

$$
\begin{array}{lll}
B_{m}^{(+)}=0, & K_{m a}^{(+)}=+\lambda_{1}^{(+)} e_{m a}, & \psi_{m 2}^{(+)}=+\alpha^{(+)} \psi_{m 1} \\
B_{m}^{(-)}=0, & K_{m a}^{(-)}=-\lambda_{1}^{(+)} e_{m a}, & \psi_{m 2}^{(-)}=-\alpha^{(+)} \psi_{m 1} .
\end{array}
$$

(The superscripts $( \pm)$ mean "evaluated on the $\mathcal{M}_{ \pm}$side of the brane $\Sigma$ ".) This is equivalent to using Eq. (9.3) on both sides of $\Sigma$ if we set $\alpha^{(-)}=-\alpha^{(+)}$and $\lambda_{1}^{(-)}=-\lambda_{1}^{(+)}$. From the expression for $\lambda_{1}$, Eq. (7.20), we see that

$$
\alpha^{(-)}=-\alpha^{(+)}, \quad q_{3}^{(-)}=-q_{3}^{(+)} \Rightarrow \lambda_{1}^{(-)}=-\lambda_{1}^{(+)} .
$$

We, therefore, find that our boundary Lagrangian $\mathcal{L}_{B}^{(\alpha)}$ is odd. (In the Mirabelli and Peskin model, the boundary Lagrangian $\mathcal{L}_{4}$ includes only even bulk fields and is itself even. Our $\mathcal{L}_{B}^{(\alpha)}$, however, includes also an odd bulk field, $\psi_{m 2}$, and odd parameters, $\alpha$ and $q_{3}$.)

The correct actions for the both sides then are

$$
S_{ \pm}=\int_{\mathcal{M}_{ \pm}} d^{5} x e_{5} \mathcal{L}_{5} \pm \int_{\partial \mathcal{M}_{ \pm}} d^{4} x e_{4} Y^{( \pm)} \pm \int_{\partial \mathcal{M}_{ \pm}} d^{4} x e_{4} \mathcal{L}_{B}^{(\alpha)( \pm)}
$$

The orbifold action is the sum of these two,

$$
S=\int_{\mathcal{M}_{+} \cup \mathcal{M}_{-}} d^{5} x e_{5} \mathcal{L}_{5}+\int_{\Sigma} d^{4} x e_{4}\left[2 Y^{(+)}\right]+\int_{\Sigma} d^{4} x e_{4}\left[2 \mathcal{L}_{B}^{(\alpha)}(+)\right]
$$

By analogy with our analysis of the Mirabelli and Peskin model [13], we expect that the $Y$-term matches onto the brane-localized terms produced by the bulk Lagrangian $\mathcal{L}_{5}$. We will show now that the match is (almost) perfect. 


\subsection{Boundary $Y$-term vs. Orbifold singular terms}

For each odd field, we can write

$$
\Phi(x, z)=\varepsilon(z) \Phi^{(+)}(x,|z|), \quad \partial_{5} \Phi=\left(\partial_{5} \Phi\right)^{(+)}+2 \Phi^{(+)} \delta(z),
$$

where $\varepsilon(z)= \pm 1$ on $\mathcal{M}_{ \pm}$. In particular,

$$
F_{m 5}=F_{m 5}^{(+)}-2 B_{m}^{(+)} \delta(z)
$$

This allows us to separate the $\Sigma$-localized terms in $\mathcal{L}_{5}$ explicitly.

The relevant part of the bulk Lagrangian is

$$
\begin{aligned}
\mathcal{L}_{5}= & -\frac{1}{2} R+\frac{i}{2} \widetilde{\Psi}_{M}^{i} \Gamma^{M 5 K} \partial_{5} \Psi_{K i} \\
& -\frac{1}{4} F_{M N} F^{M N}-\frac{1}{6 \sqrt{6}} \epsilon^{M N P Q K} F_{M N} F_{P Q} B_{K}+\ldots
\end{aligned}
$$

The analysis simplifies a lot in our gauge $\left(e_{m}^{\hat{5}}=e_{a}^{5}=0\right) .{ }^{8}$ In particular, a HUGE advantage of this gauge is that

$$
\text { there are no } \delta(z) \text {-terms in } \omega_{M A B} !
$$

Therefore,

$$
\begin{aligned}
R & =-2 e^{5 \hat{5}} e^{m a} \partial_{5} \omega_{m a \hat{5}}+\ldots \\
& =-4 e^{5 \hat{5}} e^{m a} \omega_{m a \hat{5}}^{(+)} \delta(z)+\ldots \\
& =-4 e_{\hat{5}}^{5} K^{(+)} \delta(z)+\ldots
\end{aligned}
$$

where the dots denote non-singular terms. Next,

$$
\begin{aligned}
\frac{i}{2} \widetilde{\Psi}_{M}^{i} \Gamma^{M 5 K} \partial_{5} \Psi_{K i} & =e_{\hat{5}}^{5}\left[\psi_{m 1} \sigma^{m n} \partial_{5} \psi_{n 2}+\text { h.c. }\right]+\ldots \\
& =e_{\hat{5}}^{5}\left[2 \psi_{m 1} \sigma^{m n} \psi_{n 2}^{(+)} \delta(z)+\text { h.c. }\right]+\ldots
\end{aligned}
$$

The Chern-Simons term is straightforward to consider,

$$
\begin{aligned}
\epsilon^{M N P Q K} F_{M N} F_{P Q} B_{K} & =-4 e_{\hat{5}}^{5} \epsilon^{m p q k} F_{m 5} F_{p q} B_{k}+\ldots \\
& =8 e_{\hat{5}}^{5} \epsilon^{m p q k} F_{p q} B_{k} B_{m}^{(+)} \delta(z)+\ldots \\
& =8 e_{\hat{5}}^{5} \epsilon^{m p q k} F_{p q}^{(+)} B_{k}^{(+)} B_{m}^{(+)} \varepsilon(z)^{2} \delta(z)+\ldots \\
& =0+\ldots,
\end{aligned}
$$

\footnotetext{
${ }^{8}$ Note that we keep $e_{5}^{a} \neq 0, e_{\hat{5}}^{m} \neq 0$. See Appendix D.
} 
whereas the $B_{M}$ kinetic term is the trickiest to analyze. We have

$$
-\frac{1}{4} F_{M N} F^{M N}=-\frac{1}{4} F_{m n} F^{m n}-\frac{1}{2} F_{m 5} F^{m 5} .
$$

Both terms contain $\delta(z)$-terms. Indeed, let us introduce

$$
\alpha^{m n k}=g^{m n} g^{k 5}, \quad \beta^{m n}=g^{m n} g^{55}-g^{m 5} g^{n 5} .
$$

Then

$$
F^{m 5}=\alpha^{m n k} F_{n k}+\beta^{m n} F_{n 5}, \quad F^{m n}=g^{m k} g^{n l} F_{k l}+\left(\alpha^{m k n}-\alpha^{n k m}\right) F_{k 5},
$$

and we find

$$
\begin{aligned}
-\frac{1}{4} F_{m n} F^{m n}= & \left(\alpha^{m k n} F_{m n} B_{k}\right)^{(+)} \varepsilon(z)^{2} \delta(z)+\ldots \\
-\frac{1}{2} F_{m 5} F^{m 5}= & 2 \beta^{m n} F_{m 5}^{(+)} B_{n}^{(+)} \delta(z)+\left(\alpha^{m k n} F_{m n} B_{k}\right)^{(+)} \varepsilon(z)^{2} \delta(z) \\
& -2 \beta^{m n} B_{m}^{(+)} B_{n}^{(+)} \delta(z)^{2}+\ldots
\end{aligned}
$$

Finally, combining the pieces, we obtain the following expression for the singular part of the bulk Lagrangian (the $e_{5}^{\hat{5}}$ is taken from $e_{5}=e_{4} e_{5}^{\hat{5}}$ ),

$$
\begin{aligned}
& e_{5}^{\hat{5}} \mathcal{L}_{5}=2 K^{(+)} \delta(z)+\left[2 \psi_{m 1} \sigma^{m n} \psi_{n 2}^{(+)}+\text {h.c. }\right] \delta(z) \\
& +e_{5}^{\hat{5}}\left\{2 \beta^{m n} F_{m 5}^{(+)} B_{n}^{(+)} \delta(z)+2\left(\alpha^{m k n} F_{m n} B_{k}\right)^{(+)} \varepsilon(z)^{2} \delta(z)\right. \\
& \left.-2 \beta^{m n} B_{m}^{(+)} B_{n}^{(+)} \delta(z)^{2}\right\}+\ldots
\end{aligned}
$$

This is to be compared with

$$
\begin{aligned}
2 Y^{(+)} \delta(z)= & 2 K^{(+)} \delta(z)+2\left[\psi_{m 1} \sigma^{m n} \psi_{n 2}+\text { h.c. }\right]^{(+)} \delta(z) \\
& +2 e_{5}^{\hat{5}}\left[\beta^{m n} F_{m 5} B_{n}+\alpha^{m k n} F_{m n} B_{k}\right]^{(+)} \delta(z) .
\end{aligned}
$$

\subsection{Auxiliary boundary condition}

We see that we definitely do not match the $\delta(z)^{2}$ terms. To do so, one would have to put $\delta(0)$ terms on the boundary which we consider unnatural. Instead, we refer to the discussion of the Mirabelli and Peskin model [13], where it was found that the $\delta(z)^{2}$ terms are taken care of by the auxiliary fields upon going on-shell.

How could this help if there are no auxiliary fields, but $\delta(z)^{2}$ terms are present? The point is that "going on-shell" in the boundary picture means not only eliminating the auxiliary fields, but also using some boundary conditions which are a part of the auxiliary equations of motion [13].

We conjecture that $B_{m}=0$ on $\partial \mathcal{M}$ is exactly such an "auxiliary boundary condition". (This is so if, in the $Y$-term for the off-shell supergravity action, the $B_{m}$ appears multiplied by an auxiliary field.) Using this boundary condition takes care of the discrepancy in the $\delta(z)^{2}$ terms. 


\subsection{Different $\varepsilon(z)$ for different fields}

The other mismatch is in the term with $\varepsilon(z)^{2}$,

$$
2\left(\alpha^{m k n} F_{m n} B_{k}\right)^{(+)} \varepsilon(z)^{2} \delta(z) \in \mathcal{L}_{5} .
$$

Setting $\varepsilon(z)^{2}=1$ would eliminate the discrepancy, but we are not allowed to do so, since ${ }^{9}$

$$
\varepsilon(z)^{2} \delta(z)=\frac{1}{3} \delta(z)
$$

One could find various "excuses" for neglecting this term. One could use the $B_{m}=0$ boundary condition to kill it. But this also kills the $\beta^{m n} F_{m 5} B_{n}$ term which matches perfectly. Or one could argue that it is "of higher order in the brane coupling". Indeed, this term is special in the sense that it is a product of three odd fields $\left(g^{m 5}, F_{m n}\right.$ and $\left.B_{m}\right)$ evaluated on $\partial \mathcal{M}_{+}$. If the brane action is such that these fields acquire non-zero boundary conditions, this term becomes proportional to $g^{3}$, with $g$ being a coupling constant in front of the boundary action. (This is exactly the type of expansion used by Horava and Witten in Ref. [1]. The role of $g$ is played there by $\kappa^{2 / 3}$.)

But there is, actually, another way to eliminate the mismatch. And it can be motivated as follows. Note that the orbifold construction may correspond to a discontinuous limit of some smooth supergravity realization (when the brane sources are smoothed out into the bulk). The $\varepsilon(z)$ would then correspond to a smooth warp-factor. But then, why would all the odd fields have the same warp-factor?

Let us, therefore, introduce different $\varepsilon(z)$ for different odd fields! We numerate them as follows,

$$
\begin{aligned}
& \eta_{2}=\varepsilon_{1} \eta_{2}^{(+)}, \quad \psi_{m 2}=\varepsilon_{2} \psi_{m 2}^{(+)}, \quad K_{m a}=\varepsilon_{3} K_{m a}^{(+)}, \quad q_{3}=\varepsilon_{4} q_{3}^{(+)} \\
& B_{m}=\varepsilon_{5} B_{m}^{(+)}, \quad e_{5 a}=\varepsilon_{6} e_{5 a}^{(+)}
\end{aligned}
$$

They have to satisfy $\varepsilon_{i}(z)= \pm 1$ on $\mathcal{M}_{ \pm}$, but if we have one such $\varepsilon(z)$, then we can write many functions of it ${ }^{10}$ still satisfying this property, ${ }^{11}$

$$
\varepsilon(z), \quad \frac{1}{\varepsilon(z)}, \quad \frac{2 \varepsilon(z)}{1+\varepsilon(z)^{2}}, \quad \text { etc. }
$$

\footnotetext{
${ }^{9}$ This relation was first noticed by Conrad in Ref. 25]. See also Ref. 26]. The key to its understanding is the fact that the "sign function" $\varepsilon(z)$ must be treated as a distribution, just like the delta function $\delta(z)$. One way to define it is via a limit of a sequence of regular ( $\operatorname{smooth}$ ) functions: $\varepsilon(z)=\lim \varepsilon_{n}(z)$. Accordingly, $\delta(z)=\lim \delta_{n}(z)$. The product of distributions is ill-defined unless we relate the two sequences. We require $\varepsilon_{n}^{\prime}(z)=2 \delta_{n}(z)$. Then $\lim \int d z \varepsilon_{n}^{2}(z) \delta_{n}(z) f(z)=(1 / 3) \lim \int d z \delta_{n}(z) f(z)$ for any (smooth) test function $f(z)$. This gives precise meaning to the distributional equality $\varepsilon(z)^{2} \delta(z)=(1 / 3) \delta(z)$.

${ }^{10}$ We can define a function $w(\varepsilon)$ of the distribution $\varepsilon(z)=\lim \varepsilon_{n}(z)$ by $w(\varepsilon(z))=\lim w\left(\varepsilon_{n}(z)\right)$.

${ }^{11}$ The possible appearance of the sign factors of this type in the orbifold constructions was mentioned before; see, e.g., Refs. 27, 28.
} 
The $\varepsilon(z)^{2}$ in Eq. (9.24) now changes as

$$
\varepsilon(z)^{2} \quad \longrightarrow \quad \varepsilon_{5}(z) \varepsilon_{6}(z)
$$

Therefore, by choosing $\varepsilon_{5}(z)=\varepsilon(z)$ and $\varepsilon_{6}(z)=1 / \varepsilon(z)$, or vice versa, we eliminate the mismatch!

One should be careful, however, because such a modification can change some of the relations used before. Namely, we used $\varepsilon^{\prime}(z)=2 \delta(z)$. It turns out, however, that we are safe since $^{12}$

$$
\left(\frac{1}{\varepsilon(z)}\right)^{\prime}=-\frac{1}{\varepsilon(z)^{2}} \varepsilon^{\prime}(z)=-2 \frac{1}{\varepsilon(z)^{2}} \delta(z)=2 \delta(z) .
$$

We used here the following relation,

$$
\varepsilon(z)^{-2} \delta(z)=-\delta(z)
$$

which can be proven in the same way as Eq. (9.25). Namely,

$$
\int_{-a}^{+a} \varepsilon(z)^{-2} \delta(z) d z=\frac{1}{2} \int_{-a}^{+a} \varepsilon^{-2} d \varepsilon=-\left.\frac{1}{2} \varepsilon(z)^{-1}\right|_{-a} ^{+a}=-\frac{1}{2}(1-(-1))=-1 .
$$

We will find a more convincing proof of the necessity to introduce different $\varepsilon_{i}(z)$ for different fields when checking supersymmetry of our action in the orbifold picture.

\subsection{Another addition to the $Y$-term}

We see now that the presence of the $e_{5}^{\hat{5}} F^{n 5} B_{n}$ term in the boundary action follows most easily from the requirement that the $Y$-term match singularities of the bulk Lagrangian. We will now use this approach to find another term which should be included in the $Y$-term.

In our expressions for the boundary terms of the general and supersymmetry variations of the bulk action, we ignored a contribution from the following term,

$$
-i \frac{\sqrt{6}}{16} F_{M N}\left(2 \widetilde{\Psi}^{M i} \Psi_{i}^{N}+\widetilde{\Psi}_{P}^{i} \Gamma^{M N P Q} \Psi_{Q i}\right) \in \mathcal{L}_{5}
$$

The reason was that its contribution to the boundary term of the supersymmetry variation (if it is at all non-zero) comes from $\delta_{\mathcal{H}} B_{M}$ and thus is quartic in fermions, which is of higher order than we consider. But its contribution to the boundary term of the general variation is of quadratic order in fermions and thus should be included. (Note that the variation $\delta_{\mathcal{H}} \Psi_{M i}$ in Eq. (9.33) does not contribute to the boundary term of the supersymmetry variation as the explicit calculation of $\widetilde{K}_{M}$ in Eq. (5.8) shows 115.)

\footnotetext{
${ }^{12}$ Note that although $\varepsilon^{\prime}(z)=2 \delta(z)$ and $(1 / \varepsilon(z))^{\prime}=2 \delta(z)$ are both true in the distributional sence, the functional relation $\varepsilon_{n}^{\prime}(z)=2 \delta_{n}(z)$ does not hold between $1 / \varepsilon_{n}(z)$ and $\delta_{n}(z)$.
} 
This part of $\mathcal{L}_{5}$ produces brane-localized terms because $F_{m 5} \ni-2 B_{m}^{(+)} \delta(z)$. Since the singular part of $e_{5}^{\hat{5}} \mathcal{L}_{5}$ should match onto $2 Y^{(+)} \delta(z)$, we find the following contribution to the $Y$-term,

$$
\begin{aligned}
Y^{(+)} \ni \frac{\sqrt{6}}{4} i \gamma^{m k} B_{k}^{(+)}\left(\psi_{m 2} \psi_{\hat{5} 1}-\psi_{m 1} \psi_{\hat{5} 2}\right) \\
\quad-\frac{\sqrt{6}}{8} i \varepsilon^{m n p q} B_{m}^{(+)}\left(\psi_{p 2} \sigma_{n} \bar{\psi}_{q 2}+\psi_{p 1} \sigma_{n} \bar{\psi}_{q 1}\right)+\text { h.c. }
\end{aligned}
$$

where $\psi_{\hat{5} 1,2}=e_{\hat{5}}^{5} \psi_{51,2}+e_{\hat{5}}^{m} \psi_{m 1,2}$. This contribution, however, does not change our previous analysis.

Indeed, because of the $e_{5}^{\hat{5}} F^{n 5} B_{n}$ term, the gauge invariance of the bulk-plus-boundary action is broken (on the boundary) and we have to use the $B_{m}^{(+)}=0$ boundary condition. The terms in Eq. (9.34) are, therefore, harmless for the supersymmetry variation unless we vary $B_{m}$ itself, but this is of higher order in fermions. They do modify the natural boundary conditions, making $B_{m} \sim \mathcal{O}\left(\psi^{2}\right)$, but this is again of higher order in our approximation.

Also, by construction, these terms match singularities of the bulk Lagrangian and thus do not appear in the bulk-plus-brane action of the orbifold picture.

\subsection{Result: the orbifold action}

We found that the $Y$-term of the boundary picture matches (with some subtleties) onto the brane-localized terms arising from the singularities of the bulk Lagrangian $\mathcal{L}_{5}$. As a result, the total action 9.10 reduces to

$$
S=\int_{\mathcal{M}_{5}} d^{5} x e_{5} \mathcal{L}_{5}+\int_{\Sigma} d^{4} x e_{4} \mathcal{L}_{4}
$$

where $\mathcal{M}_{5}=\mathbb{R}^{1,4}$ is the (curved) space-time without boundary, with $z \in(-\infty,+\infty)$, and $\Sigma$ denotes the brane at $z=0$. The brane Lagrangian $\mathcal{L}_{4}$ is twice the boundary Lagrangian (not including the $Y$-term) evaluated on the $\mathcal{M}_{+}$side of $\Sigma$,

$$
\mathcal{L}_{4}=2 \mathcal{L}_{B}^{(\alpha)(+)} .
$$

\section{Supersymmetry in the orbifold picture}

Here we check explicitly whether the bulk-plus-brane action, constructed starting from the boundary picture, is in fact supersymmetric in the orbifold picture. In the process, we find that using different $\varepsilon_{i}(z)$ for different odd fields is essential and that checking supersymmetry without the use of the boundary conditions fixes the $\varepsilon_{i}(z)$ uniquely.

\subsection{Bulk-plus-brane action}

Our bulk-plus-brane action is

$$
S=S_{5}+S_{4}=\int d^{5} x e_{5} \mathcal{L}_{5}+\int d^{5} x e_{4} \delta(z) \mathcal{L}_{4},
$$


where $S_{5}$ is the bulk supergravity action (5.1), and $\mathcal{L}_{4}$ is the following brane Lagrangian,

$$
\mathcal{L}_{4}=-6 \lambda_{1}+2\left[\alpha_{11} \psi_{m 1} \sigma^{m n} \psi_{n 1}+2 \alpha_{12} \psi_{m 1} \sigma^{m n} \psi_{n 2}^{(+)}+\alpha_{22} \psi_{m 2}^{(+)} \sigma^{m n} \psi_{n 2}^{(+)}+\text {h.c. }\right] .
$$

The parameters are fixed in terms of $\lambda, \vec{q}$ and $\alpha \equiv \alpha^{(+)}$,

$$
\begin{gathered}
\alpha_{11}=\frac{-\alpha}{1+\alpha \alpha^{*}}, \quad \alpha_{12}=\frac{-\alpha \alpha^{*}}{1+\alpha \alpha^{*}}, \quad \alpha_{22}=\frac{\alpha^{*}}{1+\alpha \alpha^{*}} \\
\lambda_{1}=-\frac{\alpha q_{12}^{*}+\alpha^{*} q_{12}+\left(\alpha \alpha^{*}-1\right) q_{3}^{(+)}}{1+\alpha \alpha^{*}} \lambda .
\end{gathered}
$$

This bulk-plus-brane action was derived starting from the boundary picture. (Note that setting $\alpha=0$ kills all the fermionic terms in $\mathcal{L}_{4}$. Therefore, in the orbifold picture, the transition between the $\alpha=0$ and $\alpha \neq 0$ cases is not as straightforward as in the boundary picture.)

We would now like to check explicitly that the action is supersymmetric in the orbifold picture under the local $N=2$ supersymmetry restricted on the brane by the boundary condition

$$
\eta_{2}^{(+)}=\alpha \eta_{1} \quad \text { on } \Sigma
$$

As we found in the previous section, it may be necessary to use the freedom of defining different $\varepsilon(z)$ for different fields. We therefore set

$$
\eta_{2}=\varepsilon_{1} \eta_{2}^{(+)}, \quad \psi_{m 2}=\varepsilon_{2} \psi_{m 2}^{(+)}, \quad K_{m a}=\varepsilon_{3} K_{m a}^{(+)}, \quad q_{3}=\varepsilon_{4} q_{3}^{(+)}, \quad B_{m}=\varepsilon_{5} B_{m}^{(+)}
$$

We will see that $\varepsilon_{i}$ are either $\varepsilon(z)$ or $1 / \varepsilon(z)$, so that we can freely use $\varepsilon_{i}^{\prime}(z)=2 \delta(z)$.

\subsection{Supersymmetry variation of the bulk action}

The supersymmetry variation of the bulk Lagrangian produces a total derivative term, Eq. (5.8), which was important in the boundary picture but integrates to zero on the orbifold. But on the orbifold we get additional brane-localized contributions from the bulk action due to the discontinuities in the fields and parameters.

First, we promoted the parameter $q_{3}$ to a function,

$$
q_{3}(z)=\varepsilon_{4}(z) q_{3}^{(+)}
$$

where $q_{3}^{(+)}$is a constant. Performing the supersymmetry variation of $S_{5}$ without assuming the parameters to be constant, we find [0] (dropping the total derivative term),

$$
\delta_{\mathcal{H}}^{(1)} S_{5}=\int d^{5} x e_{5}\left\{-3 i \widetilde{\Psi}_{M}^{i} \Gamma^{M N} \mathcal{H}_{j} \partial_{N}\left(\lambda Q_{i}{ }^{j}\right)-i \sqrt{6} \widetilde{\Psi}_{M}^{i} \Gamma^{M N K} \mathcal{H}_{j} B_{K} \partial_{N}\left(\lambda Q_{i}{ }^{j}\right)\right\}
$$


In our case only $\partial_{5} q_{3} \neq 0$. Going into our gauge $\left(e_{m}^{\hat{5}}=e_{a}^{5}=0\right)$, we obtain

$$
\begin{aligned}
\delta_{\mathcal{H}}^{(1)} S_{5}=\int d^{5} x e_{5} e_{\hat{5}}^{5}\left[2 \lambda q_{3}^{(+)} \delta(z)\right] & \left\{3 i\left(-\psi_{m 1} \sigma^{m} \bar{\eta}_{1}+\psi_{m 2} \sigma^{m} \bar{\eta}_{2}\right)\right. \\
+ & \left.2 \sqrt{6} i\left(\psi_{m 2} \sigma^{m n} \eta_{1}+\psi_{m 1} \sigma^{m n} \eta_{2}\right) B_{n}+\text { h.c. }\right\},
\end{aligned}
$$

where we used $q_{3}^{\prime}(z)=2 q_{3}^{(+)} \delta(z)$.

Second, because we have $\partial_{5}$ hitting odd fields in our supersymmetry transformations, the transformations are singular and thus not well-defined on the brane. In our analysis of the Mirabelli and Peskin model [13], we showed that in order for the (on-shell) supersymmetry algebra to close onto the (singular) orbifold equations of motion we need to modify the supersymmetry transformations by adding appropriate $\delta(z)$-terms. The modifications should be such that when the (natural) boundary conditions are taken into account the supersymmetry transformations become non-singular on the brane. This approach was already used in Ref. [7].

By inspection of our supersymmetry transformations, we see that $\partial_{5}$ hits $\eta_{2}$ in $\delta \psi_{52}$. Therefore, we modify the supersymmetry transformations by adding to $\delta \psi_{52}$ a new piece,

$$
\delta_{\mathcal{H}}^{(2)} \psi_{52}=-4 \eta_{2}^{(+)} \delta(z)=-4 \alpha \eta_{1} \delta(z)
$$

which subtracts the singular piece in $\delta \psi_{52}$. This modification produces an additional branelocalized contribution to the supersymmetry variation of the bulk action,

$$
\begin{aligned}
\delta_{\mathcal{H}}^{(2)} S_{5}= & \int d^{5} x e_{5} e_{\hat{5}}^{5}[-4 \delta(z)]\left\{-2 \psi_{m 1} \sigma^{m n} \widehat{D}_{n} \eta_{2}^{(+)}+i K_{n a} \psi_{m 2} \sigma^{m n} \sigma^{a} \bar{\eta}_{2}^{(+)}\right. \\
& \left.-\frac{3}{2} \lambda i\left(q_{3} \psi_{m 2}+q_{12} \psi_{m 1}\right) \sigma^{m} \bar{\eta}_{2}^{(+)}+\frac{\sqrt{6}}{4} i \gamma^{m k} F_{k \hat{5}} \psi_{m 1} \eta_{2}^{(+)}+\text {h.c. }\right\}
\end{aligned}
$$

where $\gamma^{m n}=e^{m a} e_{a}^{n}$ and $F_{m \hat{5}}=e_{\hat{5}}^{5} F_{m 5}+e_{\hat{5}}^{n} F_{m n}$. (The $F_{m n}$ terms not appearing in $F_{m \hat{5}}$, as well as the $B_{m}$ terms, are not shown here.)

All other $\partial_{5}$ in the supersymmetry transformations appear only via $F_{m 5}$. However, as we will explain in detail later, when the natural boundary condition on $B_{m}$ is $B_{m}^{(+)}=0$ on $\Sigma$, no further modifications to the supersymmetry transformations are necessary.

We will return to the discussion of the $B_{m}$ and $F_{m n}$ terms in the next section. For now we simply set $B_{m}=0$ and $F_{m n}=0$. (But we will keep $F_{m 5}$.)

\subsection{Supersymmetry variation of the brane action}

The supersymmetry variation of the brane action gives

$$
\begin{array}{r}
\delta_{\mathcal{H}} S_{4}=\int d^{5} x e_{4} \delta(z)\left\{-6 \lambda_{1} e_{a}^{m} \delta_{\mathcal{H}} e_{m}^{a}+4\left[\left(\alpha_{11} \psi_{m 1}+\alpha_{12} \psi_{m 2}^{(+)}\right) \sigma^{m n} \delta_{\mathcal{H}} \psi_{n 1}\right.\right. \\
\left.\left.+\left(\alpha_{22} \psi_{m 2}^{(+)}+\alpha_{12} \psi_{m 1}\right) \sigma^{m n} \delta_{\mathcal{H}} \psi_{n 2}^{(+)}+\text {h.c. }\right]\right\} .
\end{array}
$$


The (induced on the brane) supersymmetry transformations are

$$
\begin{gathered}
\delta_{\mathcal{H}} e_{m}^{a}=-i\left(\psi_{m 1} \sigma^{a} \bar{\eta}_{1}+\varepsilon_{1} \varepsilon_{2} \psi_{m 2}^{(+)} \sigma^{a} \bar{\eta}_{2}^{(+)}\right)+h . c . \\
\delta_{\mathcal{H}} \psi_{m 1}=2 \widehat{D}_{m} \eta_{1}+i \varepsilon_{1} \varepsilon_{3} K_{m a}^{(+)} \bar{\eta}_{2}^{(+)}+i \lambda \sigma_{m}\left(q_{12}^{*} \bar{\eta}_{1}+\varepsilon_{1} \varepsilon_{4} q_{3}^{(+)} \bar{\eta}_{2}^{(+)}\right) \\
-\frac{4 i}{2 \sqrt{6}}\left(\sigma_{m}^{n}+\delta_{m}^{n}\right) \eta_{1} F_{n \hat{5}} \\
\delta_{\mathcal{H}} \psi_{m 2}^{(+)}=2 \widehat{D}_{m} \eta_{2}^{(+)}-i K_{m a}^{(+)} \bar{\eta}_{1}+i \lambda \sigma_{m}\left(q_{3}^{(+)} \bar{\eta}_{1}-q_{12} \bar{\eta}_{2}^{(+)}\right) \\
-\frac{4 i}{2 \sqrt{6}}\left(\sigma_{m}^{n}+\delta_{m}^{n}\right) \eta_{2}^{(+)} F_{n \hat{5}},
\end{gathered}
$$

where the $\varepsilon_{i}(z)$ factors have been explicitly shown. (Note that the variations of the even fields contain products of $\varepsilon_{i}$, whereas the variation of $\psi_{m 2}^{(+)}$contains no $\varepsilon_{i}$ and simply corresponds to evaluating the bulk transformation of the odd field on the positive side of the brane.)

\subsection{Supersymmetry variation of the total action}

Writing all the $\varepsilon_{i}(z)$ factors explicitly in the expressions for the supersymmetry variation of the bulk action, we find

$$
\delta_{\mathcal{H}}^{(1)} S_{5}=\int d^{5} x e_{4} \delta(z)\left\{6 i \lambda q_{3}^{(+)}\left(-\psi_{m 1} \sigma^{m} \bar{\eta}_{1}+\varepsilon_{1} \varepsilon_{2} \psi_{m 2}^{(+)} \sigma^{m} \bar{\eta}_{2}^{(+)}\right)+\text {h.c. }\right\}
$$

and

$$
\begin{gathered}
\delta_{\mathcal{H}}^{(2)} S_{5}=\int d^{5} x e_{4} \delta(z)\left\{8 \psi_{m 1} \sigma^{m n} \widehat{D}_{n} \eta_{2}^{(+)}-4 i \varepsilon_{2} \varepsilon_{3} K_{n a}^{(+)} \psi_{m 2}^{(+)} \sigma^{m n} \sigma^{a} \bar{\eta}_{2}^{(+)}\right. \\
+6 \lambda i\left(\varepsilon_{2} \varepsilon_{4} q_{3}^{(+)} \psi_{m 2}^{(+)}+q_{12} \psi_{m 1}\right) \sigma^{m} \bar{\eta}_{2}^{(+)} \\
\left.-\sqrt{6} i \gamma^{m k} F_{k 5} \psi_{m 1} \eta_{2}^{(+)}+\text {h.c. }\right\}
\end{gathered}
$$

Adding all three contributions and setting $\eta_{2}^{(+)}=\alpha \eta_{1}$, we obtain the following expression for the supersymmetry variation of our bulk-plus-brane action,

$$
\begin{aligned}
\delta_{\mathcal{H}} S=\delta_{\mathcal{H}}^{(1)} S_{5}+ & \delta_{\mathcal{H}}^{(2)} S_{5}+\delta_{\mathcal{H}} S_{4}=\int d^{5} x e_{4} \delta(z)\left\{8\left[\widetilde{C}_{1} \psi_{m 1}+\widetilde{C}_{2} \psi_{m 2}^{(+)}\right] \sigma^{m n} \widehat{D}_{n} \eta_{1}\right. \\
+4 i K_{n a}^{(+)} & {\left[\widetilde{C}_{3} \psi_{m 1}+\widetilde{C}_{4} \psi_{m 2}^{(+)}\right] \sigma^{m n} \sigma^{a} \bar{\eta}_{1}-6 i\left[\widetilde{C}_{5} \psi_{m 1}+\widetilde{C}_{6} \psi_{m 2}^{(+)}\right] \sigma^{m} \bar{\eta}_{1} } \\
& \left.-\sqrt{6} i \gamma^{m k} F_{k \hat{5}}\left[\widetilde{C}_{1} \psi_{m 1}+\widetilde{C}_{2} \psi_{m 2}^{(+)}\right] \eta_{1}+\text { h.c. }\right\},
\end{aligned}
$$

where the coefficients are

$$
\begin{aligned}
& \widetilde{C}_{1}=\alpha_{11}+\alpha\left(\alpha_{12}+1\right) \\
& \widetilde{C}_{2}=\alpha_{12}+\alpha \alpha_{22} \\
& \widetilde{C}_{3}=\varepsilon_{1} \varepsilon_{3} \alpha_{11} \alpha^{*}-\alpha_{12} \\
& \widetilde{C}_{4}=\alpha^{*}\left(\varepsilon_{1} \varepsilon_{3} \alpha_{12}-\varepsilon_{2} \varepsilon_{3}\right)-\alpha_{22} \\
& \widetilde{C}_{5}=-\lambda_{1}+\lambda \widetilde{A}_{1} \\
& \widetilde{C}_{6}=-\varepsilon_{1} \varepsilon_{2} \lambda_{1} \alpha^{*}+\lambda \widetilde{A}_{2}
\end{aligned}
$$


with

$$
\begin{aligned}
& \widetilde{A}_{1}=\alpha_{11}\left(\varepsilon_{1} \varepsilon_{4} q_{3}^{(+)} \alpha^{*}+q_{12}^{*}\right)+\left(\alpha_{12}+1\right)\left(q_{3}^{(+)}-q_{12} \alpha^{*}\right) \\
& \widetilde{A}_{2}=\alpha_{12}\left(\varepsilon_{1} \varepsilon_{4} q_{3}^{(+)} \alpha^{*}+q_{12}^{*}\right)+\alpha_{22}\left(q_{3}^{(+)}-q_{12} \alpha^{*}\right)-\left(\varepsilon_{1} \varepsilon_{2}+\varepsilon_{2} \varepsilon_{4}\right) q_{3}^{(+)} \alpha^{*} .
\end{aligned}
$$

The total action is supersymmetric (subject only to the $\eta_{2}=\alpha \eta_{1}$ and $B_{m}=0$ boundary conditions) if all $\widetilde{C}_{i}$ vanish. Comparing $\widetilde{C}_{i}$ with $C_{i}$ in Eq. (7.15), we see that this happens if and only if

$$
\varepsilon_{1} \varepsilon_{3}=1, \quad \varepsilon_{2} \varepsilon_{3}=-1, \quad \varepsilon_{1} \varepsilon_{2}=1, \quad \varepsilon_{1} \varepsilon_{4}=1, \quad \varepsilon_{1} \varepsilon_{2}+\varepsilon_{2} \varepsilon_{4}=0 .
$$

But this must be true when multiplied by $\delta(z)$ ! Since we know that

$$
\varepsilon^{2} \delta(z)=\frac{1}{3} \delta(z), \quad \varepsilon^{-2} \delta(z)=-\delta(z)
$$

we see that our bulk-plus-brane action is supersymmetric provided we choose

$$
\varepsilon_{1}=\varepsilon(z), \quad \varepsilon_{2}=\varepsilon_{3}=\varepsilon_{4}=\frac{1}{\varepsilon(z)} .
$$

\subsection{Connection with earlier work}

Since this assignment differs from

$$
\varepsilon_{1}=\varepsilon_{2}=\varepsilon_{3}=\varepsilon_{4}=\varepsilon(z)
$$

assumed in Ref. [7], let us reproduce that calculation in which the boundary conditions

$$
K_{m a}^{(+)}=\lambda e_{m a}, \quad \psi_{m 2}^{(+)}=\alpha \psi_{m 1} \quad \text { on } \Sigma
$$

were used in checking supersymmetry of the bulk-plus-brane action.

If we use these boundary conditions in the supersymmetry variation of the action, we find

$$
\begin{gathered}
\delta_{\mathcal{H}} S=\int d^{5} x e_{4} \delta(z)\left\{\left(\widetilde{C}_{1}+\alpha \widetilde{C}_{2}\right)\left[8 \psi_{m 1} \sigma^{m n} \widehat{D}_{n} \eta_{1}-\sqrt{6} i \gamma^{m k} F_{k \hat{5}} \psi_{m 1} \eta_{1}\right]\right. \\
\left.+6 i \widetilde{M} \psi_{m 1} \sigma^{m} \bar{\eta}_{1}+\text { h.c. }\right\}
\end{gathered}
$$

where

$$
\widetilde{M}=-\lambda_{1}\left(\widetilde{C}_{3}+\alpha \widetilde{C}_{4}\right)-\left(\widetilde{C}_{5}+\lambda \widetilde{C}_{6}\right)
$$

We already saw in Eq. (7.24) that using the $\psi_{m 2}$ boundary condition in the brane action reduces the coefficients $\alpha_{i j}$ as follows,

$$
\left(\alpha_{11}, \alpha_{12}, \alpha_{22}\right) \quad \longrightarrow \quad(-\alpha, 0,0) .
$$


This makes

$$
\begin{aligned}
& \widetilde{C}_{1}=\widetilde{C}_{2}=0, \quad \widetilde{C}_{3}=-\varepsilon_{1} \varepsilon_{3} \alpha \alpha^{*}, \quad \widetilde{C}_{4}=-\varepsilon_{2} \varepsilon_{3} \alpha^{*} \\
& \widetilde{C}_{5}=-\lambda_{1}-\lambda \alpha\left(\varepsilon_{1} \varepsilon_{4} q_{3} \alpha^{*}+q_{12}^{*}\right)+\lambda\left(q_{3}-q_{12} \alpha^{*}\right) \\
& \widetilde{C}_{6}=-\varepsilon_{1} \varepsilon_{2} \lambda_{1} \alpha^{*}-\lambda\left(\varepsilon_{1} \varepsilon_{2}+\varepsilon_{2} \varepsilon_{4}\right) q_{3} \alpha^{*}
\end{aligned}
$$

and, therefore,

$$
\begin{aligned}
\widetilde{M}=\lambda_{1}[1 & \left.+\left(\varepsilon_{1} \varepsilon_{2}+\varepsilon_{1} \varepsilon_{3}+\varepsilon_{2} \varepsilon_{3}\right) \alpha \alpha^{*}\right] \\
& +\lambda\left\{q_{12} \alpha^{*}+q_{12}^{*} \alpha+q_{3}\left[\left(\varepsilon_{1} \varepsilon_{4}+\varepsilon_{1} \varepsilon_{2}+\varepsilon_{2} \varepsilon_{4}\right) \alpha \alpha^{*}-1\right]\right\} .
\end{aligned}
$$

The action is supersymmetric provided $\widetilde{M} \delta(z)=0$, which is equivalent to

$$
\begin{aligned}
& \left(\varepsilon_{1} \varepsilon_{2}+\varepsilon_{1} \varepsilon_{3}+\varepsilon_{2} \varepsilon_{3}\right) \delta(z)=\delta(z) \\
& \left(\varepsilon_{1} \varepsilon_{4}+\varepsilon_{1} \varepsilon_{2}+\varepsilon_{2} \varepsilon_{4}\right) \delta(z)=\delta(z),
\end{aligned}
$$

when Eq. (10.4) is taken into account. We see that both choices of $\varepsilon_{i}(z)$, Eqs. (10.21) and (10.22), satisfy these conditions!

We, therefore, conclude that checking supersymmetry with the boundary conditions taken into account is insufficient to distinguish between the different $\varepsilon_{i}(z)$ assignments. Without the use of the boundary conditions, supersymmetry of the bulk-plus-brane action provides more consistency checks and requires the assignment in Eq. 10.21). Namely,

$$
\eta_{2}=\varepsilon(z) \eta_{2}^{(+)}, \quad \psi_{m 2}=\frac{1}{\varepsilon(z)} \psi_{m 2}^{(+)}, \quad K_{m a}=\frac{1}{\varepsilon(z)} K_{m a}^{(+)}, \quad q_{3}=\frac{1}{\varepsilon(z)} q_{3}^{(+)}
$$

\section{Fate of $B_{m}$ terms on the orbifold}

In this section we will write down the $B_{m}$ and $F_{m n}$ terms appearing in the supersymmetry variation of the bulk-plus-brane action explicitly. We will find that they generally do not cancel so that the use of the $B_{m}^{(+)}=0$ boundary condition appears to be necessary for supersymmetry.

\section{1 $F_{m n}$ terms in the supersymmetry variation}

We consider first the $F_{m n}$ terms. Those that appear in the supersymmetry transformations and in the bulk action in the combination $F_{m \hat{5}}=e_{\hat{5}}^{5} F_{m 5}+e_{\hat{5}}^{n} F_{m n}$, go through the supersymmetry variation in this combination and cancel just as the $F_{m 5}$ terms we considered in the previous section. We will, therefore, omit them here. Among the remaining $F_{m n}$ terms, we need only the following terms in the bulk Lagrangian,

$$
\mathcal{L}_{5}=i \frac{\sqrt{6}}{8} \varepsilon^{m n p q} F_{p q}\left(\psi_{m 2} \sigma_{n} \bar{\psi}_{52}\right) e_{\hat{5}}^{5}+\text { h.c. }
$$


and only the following terms in the supersymmetry transformations,

$$
\begin{aligned}
& \delta_{\mathcal{H}} \psi_{m 1}=+\frac{1}{2 \sqrt{6}}\left(i \epsilon_{m}{ }^{n k l} \sigma_{l}+4 \delta_{m}^{n} \sigma^{k}\right) \bar{\eta}_{2} F_{n k} \\
& \delta_{\mathcal{H}} \psi_{m 2}=-\frac{1}{2 \sqrt{6}}\left(i \epsilon_{m}{ }^{n k l} \sigma_{l}+4 \delta_{m}^{n} \sigma^{k}\right) \bar{\eta}_{1} F_{n k} .
\end{aligned}
$$

There are no " $q_{3} \cdot F_{M N}$ " terms, thus $\delta_{\mathcal{H}}^{(1)} S_{5}=0$. The modification $\delta_{\mathcal{H}}^{(2)} \psi_{52}=-4 \eta_{2}^{(+)} \delta(z)$ gives

$$
\delta_{\mathcal{H}}^{(2)} S_{5}=\int d^{5} x e_{4} \delta(z)\left[-i \frac{\sqrt{6}}{2} \varepsilon^{m n p q} F_{p q}\left(\psi_{m 2} \sigma_{n} \bar{\eta}_{2}^{(+)}\right)+h . c .\right] .
$$

Using a $\sigma$-matrix identity $\left(\gamma^{m n} \equiv e_{a}^{m} e^{n a}\right)$,

$$
\sigma^{m p}\left(i \epsilon_{p}^{n k l} \sigma_{l}+4 \delta_{p}^{n} \sigma^{k}\right)=\frac{3}{2} i \epsilon^{m n k l} \sigma_{l}+\left(\gamma^{k m} \sigma^{n}-\gamma^{k n} \sigma^{m}\right),
$$

we find the following expression for the supersymmetry variation of the brane action,

$$
\begin{gathered}
\delta_{\mathcal{H}} S_{4}=\int d^{5} x e_{4} \delta(z) \frac{4}{2 \sqrt{6}}\left\{\left(\alpha_{11} \psi_{m 1}+\alpha_{12} \psi_{m 2}^{(+)}\right)\left(\frac{3}{2} i \epsilon^{m n k l} \sigma_{l}+\gamma^{k m} \sigma^{n}\right) \bar{\eta}_{2} F_{n k}\right. \\
\left.-\left(\alpha_{12} \psi_{m 1}+\alpha_{22} \psi_{m 2}^{(+)}\right)\left(\frac{3}{2} i \epsilon^{m n k l} \sigma_{l}+\gamma^{k m} \sigma^{n}\right) \bar{\eta}_{1} F_{n k}^{(+)}\right\}+ \text {h.c. }
\end{gathered}
$$

Employing the $\varepsilon_{i}(z)$ assignments and using the $\eta_{2}^{(+)}=\alpha \eta_{1}$ boundary condition, we obtain the following expression for the supersymmetry variation of our bulk-plus-brane action (showing only the $F_{m n}$ terms),

$$
\begin{array}{r}
\delta_{\mathcal{H}} S=\int d^{5} x e_{4} \delta(z) \frac{4}{2 \sqrt{6}} F_{n k}^{(+)}\left\{\frac{3}{2} i \epsilon^{m n k l}\left[Z_{1} \psi_{m 1}+Z_{2} \psi_{m 2}^{(+)}\right] \sigma_{l} \bar{\eta}_{1}\right. \\
\left.+\gamma^{k m}\left[Z_{3} \psi_{m 1}+Z_{4} \psi_{m 2}^{(+)}\right] \sigma^{n} \bar{\eta}_{1}\right\}+ \text { h.c. }
\end{array}
$$

where

$$
\begin{aligned}
& Z_{1}=\varepsilon_{1} \varepsilon_{5} \alpha_{11} \alpha^{*}-\alpha_{12}, \quad Z_{2}=\varepsilon_{1} \varepsilon_{5} \alpha_{12} \alpha^{*}-\alpha_{22}-\varepsilon_{2} \varepsilon_{5} \alpha^{*} \\
& Z_{3}=\varepsilon_{1} \varepsilon_{5} \alpha_{11} \alpha^{*}-\alpha_{12}, \quad Z_{4}=\varepsilon_{1} \varepsilon_{5} \alpha_{12} \alpha^{*}-\alpha_{22} .
\end{aligned}
$$

We find that we can make $Z_{1}=Z_{2}=Z_{3}=0$ by choosing

$$
\varepsilon_{5}=\frac{1}{\varepsilon(z)} \quad \Longleftrightarrow \quad B_{m}=\frac{1}{\varepsilon(z)} B_{m}^{(+)} .
$$

But we are still left with $Z_{4}=-\alpha^{*}$. In order to cancel the remaining piece in the supersymmetry variation,

$$
\delta_{\mathcal{H}} S=\int d^{5} x e_{4} \delta(z)\left\{-\frac{4}{2 \sqrt{6}} \alpha^{*} \gamma^{k m} F_{n k}^{(+)}\left(\psi_{m 2}^{(+)} \sigma^{n} \bar{\eta}_{1}\right)\right\}+\text { h.c. }
$$

we have to use the boundary condition $B_{m}^{(+)}=0$ on $\Sigma$. We note, however, that we need it here only in its (seemingly) gauge invariant form: $F_{n k}^{(+)}=0$ on $\Sigma$. 


\section{2 $B_{m}$ terms in the supersymmetry variation}

Repeating the above steps, we find that the $B_{m}$ terms give the following contributions,

$$
\begin{aligned}
& \delta_{\mathcal{H}}^{(1)} S_{5}=\int d^{5} x e_{4} \delta(z)\left\{4 \sqrt{6} i \lambda q_{3}^{(+)}\left(\psi_{m 2} \sigma^{m n} \eta_{1}+\psi_{m 1} \sigma^{m n} \eta_{2}\right) B_{n}+\text { h.c. }\right\} \\
& \delta_{\mathcal{H}}^{(2)} S_{5}=\int d^{5} x e_{4} \delta(z)\left\{4 \sqrt{6} i \lambda B_{n}\left[q_{3} \psi_{m 1}-q_{12}^{*}\right] \psi_{m 1} \sigma^{m n} \eta_{2}^{(+)}+h . c .\right\} \\
& \delta_{\mathcal{H}} S_{4}= \int d^{5} x e_{4} \delta(z) 4 \sqrt{6} i \lambda\left\{\left(\alpha_{11} \psi_{m 1}+\alpha_{12} \psi_{m 2}^{(+)}\right) \sigma^{m n}\left(q_{12}^{*} \eta_{2}-q_{3} \eta_{1}\right) B_{n}\right. \\
&\left.-\left(\alpha_{12} \psi_{m 1}+\alpha_{22} \psi_{m 2}^{(+)}\right) \sigma^{m n}\left(q_{3}^{(+)} \eta_{2}^{(+)}+q_{12} \eta_{1}\right) B_{n}^{(+)}\right\}+ \text {h.c. }
\end{aligned}
$$

The total contribution to the supersymmetry variation of our bulk-plus-brane action is the sum of these three,

$$
\delta_{\mathcal{H}} S=\int d^{5} x e_{4} \delta(z) 4 \sqrt{6} i \lambda B_{n}^{(+)}\left\{W_{1} \psi_{m 1} \sigma^{m n} \eta_{1}+W_{2} \psi_{m 2}^{(+)} \sigma^{m n} \eta_{1}+h . c .\right\}
$$

where

$$
\begin{aligned}
& W_{1}=\alpha \alpha_{11} q_{12}^{*}+\alpha_{12} q_{12}+q_{3}^{(+)}\left[\varepsilon_{1} \varepsilon_{5} \alpha+\varepsilon_{4} \varepsilon_{5} \alpha-\varepsilon_{4} \varepsilon_{5} \alpha_{11}+\alpha \alpha_{12}\right] \\
& W_{2}=\alpha \alpha_{12} q_{12}^{*}+\alpha_{22} q_{12}+q_{3}^{(+)}\left[\varepsilon_{2} \varepsilon_{5}-\varepsilon_{4} \varepsilon_{5} \alpha_{12}+\alpha \alpha_{22}\right] .
\end{aligned}
$$

With our $\varepsilon_{i}(z)$ assignments,

$$
\varepsilon_{1}=\varepsilon(z), \quad \varepsilon_{2}=\varepsilon_{3}=\varepsilon_{4}=\varepsilon_{5}=\frac{1}{\varepsilon(z)},
$$

the coefficients simplify to

$$
\begin{aligned}
& W_{1}=\alpha \alpha_{11} q_{12}^{*}+\alpha_{12} q_{12}-\alpha q_{3}^{(+)} \\
& W_{2}=\alpha \alpha_{12} q_{12}^{*}+\alpha_{22} q_{12}-q_{3}^{(+)}
\end{aligned}
$$

and can also be rewritten as

$$
\begin{aligned}
& W_{1}=\frac{-\alpha}{1+\alpha \alpha^{*}}\left[\left(\alpha q_{12}^{*}+q_{3}\right)+\alpha^{*}\left(q_{12}+\alpha q_{3}\right)\right] \\
& W_{2}=\frac{1}{1+\alpha \alpha^{*}}\left[\left(\alpha^{*} q_{12}-q_{3}\right)-\alpha \alpha^{*}\left(\alpha q_{12}^{*}+q_{3}\right)\right] .
\end{aligned}
$$

These coefficients do not vanish unless $\alpha=0$ and $q_{3}^{(+)}=0$ (or $\lambda=0$ for any $\alpha$ ). In a general case, we need to use the $B_{m}^{(+)}=0$ boundary condition to cancel this part of the supersymmetry variation.

This completes our check of supersymmetry of the bulk-plus-brane action, Eq. (10.1). (We remind that we work only to quadratic order in fermions.) We found that besides the boundary condition on the supersymmetry parameter, $\eta_{2}^{(+)}=\alpha \eta_{1}$, we need to use only one other boundary condition: $B_{m}^{(+)}=0$.

In order to understand if the use of this boundary condition is forced on us by the supersymmetry algebra, we have to understand when the $U(1)$ gauge invariance is broken. We discuss this in the next section. 


\section{Gauge transformations on the orbifold}

In this section we discuss the breaking of the general coordinate and the $U(1)$ gauge invariances on the orbifold. We come to the conclusion that in order to reproduce the results of the boundary picture discussion, we need to modify all the gauge transformations on the orbifold by making them non-singular! We show how the supersymmetry transformations are modified for a general $B_{m}$ boundary condition, and find that the algebra of the modified transformations closes as in the boundary picture.

\subsection{Breaking of the general coordinate invariance}

The variation of the bulk Lagrangian under the general coordinate transformation is a total derivative,

$$
\delta_{v} \mathcal{L}_{5}=D_{M}\left(v^{M} \mathcal{L}_{5}\right)
$$

In the boundary picture, this produces a boundary term, Eq. (3.1), the vanishing of which requires $v^{5}=0$ on the boundary, and in turn leads to the restriction $\eta_{2}=\alpha \eta_{1}$ on the supersymmetry parameters. But on the orbifold the total derivative integrates to zero! And it appears that the restriction $\left(v^{5}\right)^{(+)}=0$ on the brane does not arise.

If we take the point of view that the orbifold picture should reproduce all the major results of the boundary picture (such as the breaking of a gauge invariance), we are forced to make some modifications.

The necessary modification comes naturally from the requirement that the gauge transformations be the same in the both pictures both in the bulk and on the brane/boundary. But if we take the transformations of the boundary picture and assume them to be literally the same on the orbifold, we find that they are in general singular (and thus not well-defined) on the brane! Indeed,

$$
\begin{aligned}
\delta_{v} B_{m} & =v^{n} \partial_{n} B_{m}+v^{5} \partial_{5} B_{m}+B_{n} \partial_{m} v^{n}+B_{5} \partial_{m} v^{5}=2 v^{5} B_{m}^{(+)} \delta(z)+\ldots \\
\delta_{v} B_{5} & =v^{n} \partial_{n} B_{5}+v^{5} \partial_{5} B_{5}+B_{n} \partial_{5} v^{n}+B_{5} \partial_{5} v^{5}=2\left(v^{5}\right)^{(+)} B_{5} \delta(z)+\ldots,
\end{aligned}
$$

where the dots represent non-singular terms. (We used here the fact that on the orbifold $v^{5}$ is odd, whereas $v^{m}$ is even.) In order for the transformations in the both pictures to agree on the boundary, we have to modify the transformations for the orbifold picture by subtracting the singular pieces! For the $B_{M}$ field, the modified general coordinate transformations are as follows,

$$
\begin{aligned}
\delta_{v}^{\prime} B_{m} & =\delta_{v} B_{m}-2 v^{5} B_{m}^{(+)} \delta(z) \\
\delta_{v}^{\prime} B_{5} & =\delta_{v} B_{5}-2\left(v^{5}\right)^{(+)} B_{5} \delta(z) .
\end{aligned}
$$

The transformations for other fields are appropriately modified. It is clear, that the variation of the bulk Lagrangian under the modified general coordinate transformation produces now additional brane-localized terms which vanish only when $\left(v^{5}\right)^{(+)}=0$ on the brane. Therefore, all the related conclusions of the boundary picture are now reproduced. 


\subsection{Breaking of the $U(1)$ gauge invariance}

In the boundary picture, the variation of the bulk Lagrangian under the $U(1)$ gauge transformation is a total derivative (arising from the Chern-Simons term),

$$
\delta_{u} \mathcal{L}_{5}=D_{K}\left[-u \frac{1}{6 \sqrt{6}} \epsilon^{M N P Q K} F_{M N} F_{P Q}\right] .
$$

It produces a boundary term, Eq. (5.5), which tells us that the bulk action is gauge invariant only if some boundary condition is imposed. Namely, $u=0$ or $F_{m n}=0$ on the boundary.

On the orbifold, the total derivative is also generated, but it integrates to zero. It turns out, however, that under the original $U(1)$ gauge transformation, Eq. (2.9),

$$
\delta_{u} B_{M}=\partial_{M} u, \quad \delta_{u} \Psi_{M i}=u \frac{\sqrt{6}}{2} \lambda Q_{i}{ }^{j} \Psi_{M j},
$$

we now do get brane-localized terms in the variation of the bulk Lagrangian.

The variation receives a new contribution on the orbifold because the modified covariant derivative in Eq. (5.2) is not covariant under the $U(1)$ gauge transformation when the parameters are not constant,

$$
\delta_{u}\left(\widetilde{D}_{M} \Psi_{N i}\right)=u \frac{\sqrt{6}}{2} \lambda Q_{i}{ }^{j}\left(\widetilde{D}_{M} \Psi_{N j}\right)+u \frac{\sqrt{6}}{2} \partial_{M}\left(\lambda Q_{i}{ }^{j}\right) \Psi_{N j} .
$$

The variation of the Lagrangian relevant in the orbifold picture is, therefore,

$$
\delta_{u} \mathcal{L}_{5}=\frac{i}{2} \widetilde{\Psi}_{M}^{i} \Gamma^{M N K}\left[u \frac{\sqrt{6}}{2} \partial_{N}\left(\lambda Q_{i}{ }^{j}\right) \Psi_{K j}\right] .
$$

Since only $q_{3}$ is not a constant, we obtain

$$
\delta_{u} S_{5}=\int d^{5} x e_{4} \delta(z) \frac{\sqrt{6}}{2} \lambda u\left[-4 i q_{3}^{(+)}\left(\psi_{m 1} \sigma^{m n} \psi_{n 2}\right)+\text { h.c. }\right] .
$$

Therefore, the bulk action is not gauge invariant if $\lambda q_{3}^{(+)} \neq 0$.

Our brane Lagrangian is $\mathcal{L}_{4}=2 \mathcal{L}_{B}^{(\alpha)}(+)$, where $\mathcal{L}_{B}^{(\alpha)}$ is the boundary Lagrangian of the boundary picture without the $Y$-term. Its variation under the $U(1)$ gauge transformation is given in Eq. (7.33). Therefore, the brane Lagrangian is by itself gauge invariant only when $\alpha=0$ (so that the Lagrangian vanishes) or when $\lambda=0$ (so that the gauge transformation does not act on the fermions).

It is easy to check that the bulk-plus-brane action is gauge invariant only when the bulk and the brane actions are separately gauge invariant. (That is the sum of the two contributions still vanishes only when $\alpha=0$ and $q_{3}^{(+)}=0$. Or when $\lambda=0$.)

On the other hand, the boundary condition $\eta_{2}^{(+)}=\alpha \eta_{1}$ is gauge invariant when

$$
\lambda\left[\left(q_{12}+\alpha q_{3}^{(+)}\right)-\alpha\left(\alpha q_{12}^{*}-q_{3}^{(+)}\right)\right]=0 .
$$


It follows that the $U(1)$ gauge invariance in the orbifold picture is broken by either the bulk-plus-brane action or the fermionic boundary condition unless $\lambda=0$ !

Since this is drastically different from the way the $U(1)$ gauge invariance is broken in the boundary picture, we have to make some modifications if we would like the two pictures to describe the same physics.

\subsection{Modified $U(1)$ gauge transformation}

We found that taking the $U(1)$ gauge transformation in the orbifold picture to be literally the same as in the boundary picture leads to very different conclusions about the breaking of the gauge invariance in the both pictures. Therefore, as in the case of the general coordinate invariance, we are led to modify the $U(1)$ gauge transformation in the orbifold picture. The modification affects only $B_{5}$,

$$
\delta_{u}^{\prime} B_{5}=\partial_{5} u-2 u^{(+)} \delta(z)
$$

(The parameter $u$ is odd.) The modified transformation is non-singular on the brane and coincides with the $U(1)$ transformation induced on the boundary in the boundary picture,

$$
\delta_{u} B_{5}=\partial_{5} u \quad \text { on } \partial \mathcal{M} \quad \Longleftrightarrow \quad \delta_{u}^{\prime} B_{5}=\partial_{5} u^{(+)} \quad \text { on } \Sigma \text {. }
$$

The variation of the bulk action under the modified $U(1)$ gauge transformation produces the following brane-localized term (from the variation of the Chern-Simons term in the bulk Lagrangian),

$$
\delta_{u}^{\prime} S_{5} \ni \int d^{5} x e_{4} \delta(z)\left\{u^{(+)} \frac{2}{6 \sqrt{6}} \epsilon^{m n p q} F_{m n} F_{p q}\right\},
$$

which is the orbifold version of Eq. (5.5).

For the covariant derivative defined in Eq. (5.2) we now obtain, instead of Eq. (12.6),

$$
\begin{aligned}
\delta_{u}\left(\widetilde{D}_{5} \Psi_{N i}\right)= & u \frac{\sqrt{6}}{2} \lambda Q_{i}{ }^{j}\left(\widetilde{D}_{5} \Psi_{N j}\right) \\
& +u \frac{\sqrt{6}}{2} \partial_{5}\left(\lambda Q_{i}{ }^{j}\right) \Psi_{N j}+\sqrt{6} u^{(+)} \delta(z)\left(\lambda Q_{i}{ }^{j}\right) \Psi_{N j} .
\end{aligned}
$$

The variation of the the fermionic part of the bulk action now gets two contributions. (Let us use the shorthand notation $\psi_{i} \psi_{j} \equiv \psi_{m i} \sigma^{m n} \psi_{n j}$ for the following.) The first contribution, arising from the jumping parameter $q_{3}=\varepsilon_{4} q_{3}^{(+)}$, is

$$
\delta_{u}^{\prime(1)} S_{5}=\int d^{5} x e_{4} \delta(z) \frac{\sqrt{6}}{2} \lambda u\left[-4 i q_{3}^{(+)} \psi_{1} \psi_{2}+\text { h.c. }\right]
$$

and the second one, arising from the extra piece $\delta_{u}^{\prime(2)} B_{5}=-2 u^{(+)} \delta(z)$ in the modified gauge transformation, is

$$
\delta_{u}^{\prime(2)} S_{5}=\int d^{5} x e_{4} \delta(z) \frac{\sqrt{6}}{2} \lambda u^{(+)}\left[-4 i q_{3} \psi_{1} \psi_{2}-2 i q_{12}\left(\psi_{1} \psi_{1}+\bar{\psi}_{2} \bar{\psi}_{2}\right)+\text { h.c. }\right] .
$$


The variation of the brane action is twice that in Eq. (7.33), with $\psi_{m 2}$ and $q_{3}$ evaluated on the $\mathcal{M}_{+}$side of $\Sigma$. Namely,

$$
\delta_{u}^{\prime} S_{4}=\int d^{5} x e_{4} \delta(z) \frac{\sqrt{6} i \lambda u^{(+)}}{1+\alpha \alpha^{*}}\left[X_{1} \psi_{1} \psi_{1}+X_{2} \psi_{1} \psi_{2}^{(+)}+X_{3} \psi_{2}^{(+)} \psi_{2}^{(+)}\right]+\text {h.c. }
$$

where

$$
X_{1}=X_{3}^{*}=2 \alpha\left(\alpha^{*} q_{12}-q_{3}^{(+)}\right), \quad X_{2}=2\left(\alpha q_{12}^{*}-\alpha^{*} q_{12}\right)
$$

The total variation of the fermionic part of the bulk-plus-brane action under the (modified) $U(1)$ gauge transformation is the sum of the three contributions,

$$
\delta_{u}^{\prime} S \ni \int d^{5} x e_{4} \delta(z) \frac{\sqrt{6} i \lambda u^{(+)}}{1+\alpha \alpha^{*}}\left[\widetilde{X}_{1} \psi_{1} \psi_{1}+\widetilde{X}_{2} \psi_{1} \psi_{2}^{(+)}+\widetilde{X}_{3} \psi_{2}^{(+)} \psi_{2}^{(+)}\right]+\text {h.c. }
$$

where

$$
\begin{aligned}
\widetilde{X}_{1} & =X_{1}-q_{12}\left(1+\alpha \alpha^{*}\right) \\
\widetilde{X}_{3} & =X_{3}+q_{12}^{*}\left(1+\alpha \alpha^{*}\right) \varepsilon_{2} \varepsilon_{2} \\
\widetilde{X}_{2} & =X_{2}-2 q_{3}^{(+)}\left(1+\alpha \alpha^{*}\right)\left[\varepsilon_{u} \varepsilon_{2}+\varepsilon_{2} \varepsilon_{4}\right]
\end{aligned}
$$

after all the $\varepsilon_{i}(z)$ factors are separated. The $\varepsilon_{u}$ is such a factor for the odd parameter $u$,

$$
u(x, z)=\varepsilon_{u}(z) u^{(+)}(x,|z|) .
$$

With our $\varepsilon_{i}(z)$ assignments, Eq. (10.21), we have $\varepsilon_{2} \varepsilon_{2} \delta(z)=-\delta(z)$. Therefore,

$$
\widetilde{X}_{1}=\widetilde{X}_{3}^{*}=\alpha\left(\alpha^{*}-q_{3}^{(+)}\right)-\left(\alpha q_{3}^{(+)}+q_{12}\right)
$$

which is exactly the coefficient $W$ in Eq. (7.30)! More than that, if we choose

$$
\varepsilon_{u}=\varepsilon(z)
$$

we get $\left[\varepsilon_{u} \varepsilon_{2}+\varepsilon_{2} \varepsilon_{4}\right] \delta(z)=0$, so that the equation (7.30) is reproduced completely! This means that the fermionic part of our bulk-plus-brane action is now gauge invariant with the same restriction on the parameters $\vec{q}$ and $\alpha$, Eq. (7.28), as is necessary for the gauge invariance of the boundary condition $\eta_{2}^{(+)}=\alpha \eta_{1}$ !

After this choice of $\vec{q}$ and $\alpha$ is made, the variation of the bulk-plus-brane action under the $U(1)$ gauge transformation has only one uncanceled piece, Eq. (12.12),

$$
\delta_{u}^{\prime} S=\int d^{5} x e_{4} \delta(z)\left\{u^{(+)} \frac{2}{6 \sqrt{6}} \epsilon^{m n p q} F_{m n} F_{p q}\right\},
$$

which is the orbifold picture analog of Eq. (5.5). Therefore, our modification of the gauge transformations in the orbifold picture, Eq. (12.10), leads to agreement with conclusions of the boundary picture. 


\subsection{Modified supersymmetry transformations}

The modification of the supersymmetry transformation for $\psi_{52}$, Eq. (10.10), now becomes just a part of the general modification of all the gauge transformations in the orbifold picture. Indeed,

$$
\delta_{\mathcal{H}}^{\prime} \psi_{52}=\delta_{\mathcal{H}} \psi_{52}-4 \eta_{2}^{(+)} \delta(z)
$$

is an analog of Eq. (12.10) which makes the supersymmetry transformations non-singular on the brane, so that the induced on the brane transformations are exactly the same as those in the boundary picture.

The supersymmetry transformations should also be modified when the boundary condition on $B_{m}$ is no longer $B_{m}^{(+)}=0$, but instead $B_{m}^{(+)}=J_{m}$. This happens, for example, when one couples the $B_{m}$ field to some brane-localized matter. The $J_{m}$ is then a composite of the brane matter fields. (The coupling of brane-localized matter to the bulk supergravity in five dimensions is discussed in Refs. [29, 30, 31, 32, 33, 34].)

The necessary modifications in the supersymmetry transformations can be obtained simply by the following substitution,

$$
F_{m 5} \quad \longrightarrow \quad F_{m 5}+2 J_{m} \delta(z)
$$

which makes the (modified) transformations non-singular when the boundary condition $B_{m}^{(+)}=$ $J_{m}$ is taken into account. (From our analysis [13] of the Mirabelli and Peskin model, we know that we need this substitution, and not $F_{m 5} \rightarrow F_{m 5}+2 B_{m}^{(+)} \delta(z)$. The reason is that the supersymmetry variations of $B_{m}^{(+)}$and $J_{m}$ are different, which plays a role when the supersymmetry algebra is calculated.)

Explicitly, the modified supersymmetry transformations are

$$
\begin{aligned}
& \delta_{\mathcal{H}}^{\prime} \psi_{m 1}=\delta_{\mathcal{H}} \psi_{m 1}-\frac{8 i}{2 \sqrt{6}}\left(\sigma_{m}{ }^{n}+\delta_{m}^{n}\right) \eta_{1} J_{n} e_{\tilde{5}}^{5} \delta(z) \\
& \delta_{\mathcal{H}}^{\prime} \psi_{m 2}=\delta_{\mathcal{H}} \psi_{m 2}-\frac{8 i}{2 \sqrt{6}}\left(\sigma_{m}{ }^{n}+\delta_{m}^{n}\right) \eta_{2} J_{n} e_{\tilde{5}}^{5} \delta(z) \\
& \delta_{\mathcal{H}}^{\prime} \psi_{51}=\delta_{\mathcal{H}} \psi_{51}-\frac{8}{2 \sqrt{6}} \sigma^{n} \bar{\eta}_{2} J_{n} \delta(z)+\frac{8 i}{2 \sqrt{6}} e_{\tilde{5}}^{m}\left(\sigma_{m}^{n}+\delta_{m}^{n}\right) \eta_{1} J_{n} \delta(z) \\
& \delta_{\mathcal{H}}^{\prime} \psi_{52}=\delta_{\mathcal{H}} \psi_{52}+\frac{8}{2 \sqrt{6}} \sigma^{n} \bar{\eta}_{1} J_{n} \delta(z)+\frac{8 i}{2 \sqrt{6}} e_{\tilde{5}}^{m}\left(\sigma_{m}{ }^{n}+\delta_{m}^{n}\right) \eta_{2} J_{n} \delta(z)-4 \eta_{2}^{(+)} \delta(z) .
\end{aligned}
$$

It remains to see whether these are the correct modifications for a particular model. What we can check at the moment is the closure of the supersymmetry algebra on the bosonic fields. 
Let us consider the commutator of the two (modified) supersymmetry transformations on $B_{5}$. We find,

$$
\begin{aligned}
{\left[\delta_{\Xi}^{\prime}, \delta_{\mathcal{H}}^{\prime}\right] B_{5}=} & i \frac{\sqrt{6}}{2}\left(\eta_{1} \delta_{\Xi}^{\prime} \psi_{52}-\eta_{2} \delta_{\Xi}^{\prime} \psi_{51}\right) \\
= & {\left[\delta_{\Xi}, \delta_{\mathcal{H}}\right] B_{5}+\left\{2 i \sqrt{6}\left(\eta_{2}^{(+)} \xi_{1}-\eta_{1} \xi_{2}^{(+)}\right) \delta(z)\right.} \\
& \left.+4 i\left(\eta_{1} \sigma^{n} \bar{\xi}_{1}+\eta_{2} \sigma^{n} \bar{\xi}_{2}\right) J_{n} \delta(z)+4\left(\eta_{2} \xi_{1}-\eta_{1} \xi_{2}\right) e_{\tilde{5}}^{n} J_{n} \delta(z)+\text { h.c. }\right\} .
\end{aligned}
$$

From the (original) supersymmetry algebra, Eq. (2.6), we know that

$$
\begin{aligned}
{\left[\delta_{\Xi}, \delta_{\mathcal{H}}\right] B_{5} } & =\delta_{v} B_{5}+\delta_{u} B_{5} \\
& =v^{n} \partial_{n} B_{5}+v^{5} \partial_{5} B_{5}+B_{n} \partial_{5} v^{n}+B_{5} \partial_{5} v^{5}+\partial_{5} u,
\end{aligned}
$$

where (see Eq. (2.10))

$$
\begin{aligned}
u & =-v^{m} B_{m}-v^{5} B_{5}+u_{0} \\
v^{m} & =2 i\left(\eta_{1} \sigma^{m} \bar{\xi}_{1}+\eta_{2} \sigma^{m} \bar{\xi}_{2}\right)+2 e_{\hat{5}}^{m}\left(\eta_{2} \xi_{1}-\eta_{1} \xi_{2}\right)+h . c . \\
u_{0} & =-i \sqrt{6}\left(\eta_{2} \xi_{1}-\eta_{1} \xi_{2}\right)+\text { h.c. }
\end{aligned}
$$

We can, therefore, write

$$
\left[\delta_{\Xi}, \delta_{\mathcal{H}}\right] B_{5}=v^{n} F_{n 5}+\partial_{5} u_{0}
$$

whereas for the commutator of the modified supersymmetry transformations we find

$$
\left[\delta_{\Xi}^{\prime}, \delta_{\mathcal{H}}^{\prime}\right] B_{5}=v^{n}\left[F_{n 5}+2 J_{n} \delta(z)\right]+\left[\partial_{5} u_{0}-2 u_{0}^{(+)} \delta(z)\right]
$$

This is one explicit check of the fact that the commutator of the modified supersymmetry transformations closes onto the modified gauge transformations. (It is, actually, obvious. The commutator of two non-singular transformations must be non-singular!)

But in order that the algebra of the modified gauge transformations close without the use of the boundary conditions, we should correct our modified general coordinate transformations, Eq. (12.3), by replacing there $B_{m}^{(+)}$with $J_{m}$ (and similarly for other odd fields). Then,

$$
\delta_{v}^{\prime} B_{m}=\delta_{v} B_{m}-2 v^{5} J_{m} \delta(z)
$$

The modified transformation is, therefore, non-singular only when the boundary condition $B_{m}^{(+)}=J_{m}$ is taken into account. If the natural boundary condition for $B_{m}$ is $B_{m}^{(+)}=0$, then no modification is necessary. 


\section{Brane-localized matter}

In this section we argue that the preservation of the bulk $U(1)$ gauge invariance is necessary if the brane-localized matter is to provide a non-zero boundary condition for the bulk $B_{m}$ field. We show that a seemingly gauge non-invariant boundary condition $B_{m}^{(+)}=J_{m}$ can in fact be gauge invariant, if the brane fields transform appropriately under the bulk $U(1)$ transformation. We also find that a similar boundary condition exists in the Horava and Witten model.

\subsection{Preserving the bulk $U(1)$ gauge invariance}

Let us discuss the addition of the brane-localized matter a little bit further. As in Section 12.4, all we will use is that the boundary condition for $B_{m}$ is modified to $B_{m}^{(+)}=J_{m}$, where $J_{m}$ is a composite of the brane-localized fields.

We found that we need to make a modification of our gauge transformations by making them non-singular. The modified transformations coincide with those of the boundary picture exactly, both in the bulk and on the brane/boundary. Therefore, all the conclusions of the boundary picture discussion hold in the orbifold picture as well. In particular, if the $U(1)$ gauge invariance is broken, then the closure of the supersymmetry algebra requires (because of Eq. 4.6) the $B_{m}^{(+)}=0$ boundary condition. But now this boundary condition is inconsistent with the natural boundary condition $B_{m}^{(+)}=J_{m}$. The only way out is to preserve the $U(1)$ gauge invariance.

We can try the following approach. In parallel with the modification of the supersymmetry transformations, we make the same substitution (12.25),

$$
F_{m 5} \longrightarrow F_{m 5}^{\prime}=F_{m 5}+2 J_{m} \delta(z),
$$

in the bulk action. The modified action then has $B_{m}^{(+)}=J_{m}$ as its natural boundary condition. Indeed, the $B_{M}$ equation of motion is now

$$
D_{M} F^{M K}-\frac{3}{6 \sqrt{6}} \epsilon^{M N P Q K} F_{M N} F_{P Q}=0
$$

(omitting the 2-Fermi terms), where $F_{m 5}$ should be replaced by $F_{m 5}^{\prime}$. The cancellation of the singular terms requires $F_{m 5}^{\prime}$ to be non-singular, which determines the jump of $B_{m}$ across the brane. The parity assignment $\left(B_{m}\right.$ is odd) then implies the boundary condition $B_{m}^{(+)}=J_{m}$.

Under the modified $U(1)$ gauge transformation, Eq. (12.10),

$$
\delta_{u}^{\prime} B_{5}=\partial_{5} u-2 u^{(+)} \delta(z)
$$

the original $F_{m 5}$ is not invariant, whereas the modified $F_{m 5}^{\prime}$ can be made invariant, if we choose a special transformation for $J_{m}$,

$$
\delta_{u}^{\prime} J_{m}=\partial_{m} u^{(+)} \Rightarrow \delta_{u}^{\prime} F_{m 5}^{\prime}=0
$$


Since the gauge transformation for $B_{m}$ is unmodified, $\delta_{u}^{\prime} B_{m}=\partial_{m} u$, its restriction on the brane is

$$
\delta_{u}^{\prime} B_{m}^{(+)}=\partial_{m} u^{(+)} \quad \text { on } \Sigma
$$

which means that the boundary condition $B_{m}^{(+)}=J_{m}$ is now gauge invariant!

But the modified bulk-plus-brane action is not yet gauge invariant. Its variation under the modified $U(1)$ gauge transformation is still given by Eq. (12.23). It appears that there is no way to cancel it, at least without the use of the boundary condition for $B_{m}$. (Note that adding any term with $B_{m}$ in the brane action would now break the construction, giving a different boundary condition for $B_{m}$.) But if we use the $B_{m}^{(+)}=J_{m}$ boundary condition, we can write Eq. (12.23) as follows,

$$
\delta_{u}^{\prime} S=\int d^{5} x e_{4} \delta(z)\left\{u^{(+)} \frac{2}{6 \sqrt{6}} \epsilon^{m n p q} J_{m n} J_{p q}\right\}
$$

where $J_{m n}=\partial_{m} J_{n}-\partial_{n} J_{m}$. This, in principle, can be canceled by the variation of the brane action (excluding terms which combine into $F_{m 5}^{\prime}=F_{m 5}+2 J_{m} \delta(z)$ ).

For example, suppose that the matter fields on the brane include a scalar $\phi$ and two vectors, $A_{m}$ and $C_{m}$. Take their transformations under the bulk $U(1)$ to be as follows,

$$
\delta_{u}^{\prime} \phi=u^{(+)}, \quad \delta_{u}^{\prime} A_{m}=\partial_{m} u^{(+)}, \quad \delta_{u}^{\prime} C_{m}=0
$$

Let the brane action (before coupling) contain $\phi$ and $A_{m}$ only via $A_{m n} \equiv \partial_{m} A_{n}-\partial_{n} A_{m}$ and $\mathcal{D}_{m} \phi=\partial_{m} \phi-A_{m}$, so that it is gauge invariant. Now couple the brane fields to the bulk supergravity in the way described above, taking

$$
J_{m}=\partial_{m} \phi+C_{m}
$$

which has the correct gauge transformation, $\delta_{u}^{\prime} J_{m}=\partial_{m} u^{(+)}$. The resulting action is not yet gauge invariant, because Eq. (13.6) gives

$$
\delta_{u}^{\prime} S=\int d^{5} x e_{4} \delta(z)\left\{u^{(+)} \frac{2}{6 \sqrt{6}} \epsilon^{m n p q} C_{m n} C_{p q}\right\}
$$

where $C_{m n} \equiv \partial_{m} C_{n}-\partial_{n} C_{m}$. But it is now easy to make it gauge invariant by adding a term of the form $\phi \epsilon^{m n p q} C_{m n} C_{p q}$ to the brane action.

We conclude, therefore, that adding brane-localized matter can help restore the invariance of the bulk-plus-brane action (and of the associated with it natural boundary conditions) under the bulk $U(1)$ gauge transformation. The preservation of this invariance is necessary for supersymmetry as we argued based on the closure of the supersymmetry algebra.

We also would like to emphasize that it appears to be impossible to maintain the gauge invariance without the use of the $B_{m}$ boundary condition. 


\subsection{Modified Bianchi identity}

The modified field strength, $F_{M N}^{\prime}$, which we introduced satisfies the following modified Bianchi identity,

$$
\left(d F^{\prime}\right)_{5 n k}=\frac{1}{3}\left(\partial_{5} F_{n k}^{\prime}+\partial_{n} F_{k 5}^{\prime}-\partial_{k} F_{n 5}^{\prime}\right)=\frac{2}{3} J_{n k} \delta(z),
$$

where $(d F)_{M N K} \equiv \partial_{[M} F_{N K]}$ and $J_{m n} \equiv \partial_{m} J_{n}-\partial_{n} J_{m}$. This is not a surprise, since our construction in the previous subsection is analogous to the construction used by Horava and Witten [1]. But while they came to the modification of the bulk field strength in order to preserve the brane-localized gauge invariance, we need it to preserve the bulk gauge invariance. And our modification is forced on us by the supersymmetry algebra!

Note that Horava and Witten write their boundary condition, Eq. (2.20) in Ref. [1], in a form which appears to be manifestly gauge invariant (under both the brane and the bulk gauge invariances). The form is analogous to our $F_{m n}^{(+)}=J_{m n}$. However, this relation is derivative from the basic boundary condition $B_{m}^{(+)}=J_{m}$. There is a similar not manifestly gauge invariant boundary condition in the Horava and Witten model.

The boundary condition $B_{m}^{(+)}=J_{m}$ follows from our (modified) action as a natural boundary condition, since now $F_{m 5}^{\prime}$ must be non-singular to avoid uncanceled singularities in the $B_{M}$ equation of motion. It is exactly what Horava and Witten say after Eq. (2.18) in Ref. [1]. Their Eq. (2.13) then implies the boundary condition $C_{A B C}^{(+)} \sim \omega_{A B C}$, from which their Eq. (2.20) follows. This boundary condition can be made invariant under the brane gauge invariance, provided that $C_{A B C}$ transforms under the brane gauge transformation like $\omega_{A B C}$ in their Eq. (2.14). However, it is unclear how to achieve the bulk gauge invariance $\left(\delta C_{I J K}=\partial_{[I} \Lambda_{J K]}\right.$ with arbitrary $\left.\Lambda_{I J}\right)$ of this boundary condition.

The boundary condition $C_{A B C}^{(+)} \sim \omega_{A B C}$ was also found in Refs. [35, 36] (although there it was not derived as a natural boundary condition following from the action, but simply imposed for consistency). The transformation of $C_{A B C}$ necessary to preserve the brane gauge invariance is provided there, but the preservation of the bulk gauge invariance is not discussed. 


\section{Summary and Conclusions}

In this paper we constructed a bulk-plus-boundary action with the five-dimensional gauged (on-shell) supergravity in the bulk which is supersymmetric upon the use of the minimum set of boundary conditions dictated by the supersymmetry algebra. In a general case when the supersymmetry parameter $\mathcal{H}_{i}$ is restricted by $\eta_{2}=\alpha \eta_{1}$ on the boundary, we found that only the boundary condition $B_{m}=0$ have to be used to prove supersymmetry to second order in fermions. Other boundary conditions following from the action, $K_{m a}=\lambda_{1} e_{m a}$ and $\psi_{m 2}=\alpha \psi_{m 1}$ on $\partial \mathcal{M}$, are not needed in the proof of supersymmetry of the action.

The necessary ingredient of our boundary action is the Gibbons-Hawking-like $Y$-term presented in Eq. (9.2),

$$
Y=K+\left(\psi_{m 1} \sigma^{m n} \psi_{n 2}+\text { h.c. }\right)+\gamma^{m n} F_{m \hat{5}} B_{n}
$$

(where we used $e_{5}^{\hat{5}} F^{m 5}=\gamma^{m n} F_{n \hat{5}}$ as follows from Eq. (D.10) ). It includes

1) the standard Gibbons-Hawking term (the trace of the extrinsic curvature) which allows the derivation of the boundary condition $K_{m a}=\lambda_{1} e_{m a}$ as a natural boundary condition corresponding to the variation $\delta e_{m a}$ [12];

2) a fermionic term, which leads to the derivation of $\psi_{m 2}=\alpha \psi_{m 1}$ as a natural boundary condition for $\delta \psi_{m 1}$;

3) another bosonic term, which lets us derive the $B_{m}=0$ boundary condition as a natural boundary condition.

We argued that the $Y$-term can be derived most easily from the fact that it must match onto the brane-localized singularities of the bulk Lagrangian. (This can be used to derive appropriate additions to the $Y$-term when higher order Fermi terms are considered.)

In the transition to the orbifold picture, the $Y$-term disappears. The rest of the boundary action becomes (after the multiplication by 2) the brane action with which supersymmetry of the bulk-plus-brane action in the orbifold picture can once again be proven using only the minimum set of the boundary conditions. We found, however, that one also has to choose unconventional $\varepsilon(z)$ assignments for the odd fields and parameters, Eqs. (10.21),

$$
\eta_{2}=\varepsilon(z) \eta_{2}^{(+)}, \quad \psi_{m 2}=\frac{1}{\varepsilon(z)} \psi_{m 2}^{(+)}, \quad K_{m a}=\frac{1}{\varepsilon(z)} K_{m a}^{(+)}, \quad q_{3}=\frac{1}{\varepsilon(z)} q_{3}^{(+)}
$$

and use the property $\varepsilon(z)^{-2} \delta(z)=-\delta(z)$.

The reason for such $\varepsilon(z)$ assignments is unclear. (Perhaps, the explanation can come from a smooth realization of the supersymmetric Randall-Sundrum scenario.) We can only observe that together with the Eq. (12.22), $u=\varepsilon(z) u^{(+)}$(where $u$ is the odd parameter of the $U(1)$ gauge transformation), there is an indication that odd parameters of local transformations come with $\varepsilon(z)$, whereas other fields and parameters come with $1 / \varepsilon(z)$. (There is a slight 
problem with such a conclusion, because the equation (11.8) and the discussion in Section 9.5 seem to indicate that

$$
B_{m}=\frac{1}{\varepsilon(z)} B_{m}^{(+)}, \quad e_{5 a}=\varepsilon(z) e_{5 a}^{(+)}
$$

But the evidence provided for these assignments is not on a very firm footing.)

Another important conclusion of this work is that in the orbifold picture all local transformations have to be modified by the addition of brane-localized terms. The modifications must be such that the modified transformations become non-singular on the brane when the natural boundary conditions (encoded in the action) are used. This is the reason both for the modification of $\delta_{\mathcal{H}} \psi_{52}$ in Refs. [ [ [, ]] and for the "modification of the Bianchi identity" in the Horava-Witten model [1].

We also note that our results (concerning the boundary picture) are in agreement with the recent work of Moss 35, 36, who did a similar analysis for the eleven-dimensional supergravity. The use of the fermionic boundary condition there is necessary, according to our discussion, precisely because higher order fermionic terms are considered. Our approach to the $Y$-term can be employed there to derive the boundary condition for $C_{A B C}$ from the bulk-plus-boundary action (instead of just postulating it for consistency).

Finally, it would be interesting to see how the analysis presented here for the case of on-shell supergravity can be done for off-shell supergravity of Zucker [18]. One question this could answer is whether the boundary condition $B_{m}=0$ is, actually, an "auxiliary boundary condition" (see Section 9.4) similar to $\Phi=0$ in the Mirabelli and Peskin model (in the absence of brane-localized matter) 13.

\section{Acknowledgments}

I would like to thank Jonathan Bagger for his interest in this work, many helpful discussions and critical reading of the manuscript. This work was supported in part by the National Science Foundation, grant NSF-PHY-0401513. 


\section{A. Conventions}

We follow conventions of Ref. [7]. Our indices are

$$
\begin{array}{cccc}
M, N, P, Q, K & \text { curved space } & M=\{m, 5\} & m=\{0,1,2,3\} \\
A, B, C, D, E & \text { tangent space } & A=\{a, \hat{5}\} & a=\{\hat{0}, \hat{1}, \hat{2}, \hat{3}\} \\
i, j & S U(2) & i=\{1,2\} . &
\end{array}
$$

We denote the determinant of an $n$-bein by $e_{n}: e_{5}=\operatorname{det} e_{M}^{A}, e_{4}=\operatorname{det} e_{m}^{a}$. We use the fünfbein $e_{M}^{A}$ to relate the two types of indices, e.g.

$$
g_{M N}=e_{M}^{A} e_{N}^{B} \eta_{A B}, \quad \epsilon^{M N P Q K}=e_{A}^{M} e_{B}^{N} e_{C}^{P} e_{D}^{Q} e_{E}^{K} \epsilon^{A B C D E} .
$$

It also defines the torsion-free connection,

$$
\omega(e)_{M A B}=\frac{1}{2} e_{A}^{N} e_{B}^{K}\left(C_{M N K}+C_{N M K}-C_{K M N}\right),
$$

where $C_{M N K}=e_{M C}\left(\partial_{N} e_{K}^{C}-\partial_{K} e_{N}^{C}\right)$. The covariant derivative is defined to act as follows,

$$
D(\omega)_{M} \Psi_{N}^{A}=\partial_{M} \Psi_{N}^{A}+\omega_{M C} \Psi_{N}^{C}-\Gamma(\omega)_{M N}^{K} \Psi_{K}^{A}+\frac{1}{4} \omega_{M B C} \Gamma^{B C} \Psi_{N}^{A},
$$

where the spinor indices on $\Psi$ and $\Gamma^{B C}$ are implicit. The Christoffel connection is made dependent by imposing $D_{M} e_{N}^{A}=0$, which implies

$$
\Gamma_{M N}^{K}=\omega_{M N}^{K}+e_{A}^{K} \partial_{M} e_{N}^{A} .
$$

The curvature tensor is defined by

$$
R_{M N A B}=\partial_{M} \omega_{N A B}-\partial_{N} \omega_{M A B}+\omega_{N A}{ }^{C} \omega_{M C B}-\omega_{M A}{ }^{C} \omega_{N C B},
$$

and the scalar curvature is $R=e^{M A} R_{M A}=e^{M A} e^{N B} R_{M N A B}$.

The gamma matrices obey the following relations,

$$
\begin{gathered}
\left\{\Gamma^{A}, \Gamma^{B}\right\}=-2 \eta^{A B}, \quad \Gamma^{A B C D E}=-\epsilon^{A B C D E} \\
\Gamma^{A B C D}=\epsilon^{A B C D E} \Gamma_{E}, \quad \Gamma^{A B C}=\frac{1}{2} \epsilon^{A B C D E} \Gamma_{D E},
\end{gathered}
$$

where $\Gamma^{A_{1} \ldots A_{n}}$ are antisymmetrized with "strength one", e.g. $\Gamma^{A B}=\frac{1}{2}\left(\Gamma^{A} \Gamma^{B}-\Gamma^{B} \Gamma^{A}\right)$. The metric and the Levi-Civita tensor are determined by

$$
\eta_{A B}=\operatorname{diag}(-++++), \quad \epsilon^{\hat{0} \hat{1} \hat{2} \hat{3} \hat{5}}=+1, \quad \epsilon^{a b c d \hat{5}}=\epsilon^{a b c d} .
$$

In reduction to the two-component notation [37] we use the following representation of the gamma matrices,

$$
\begin{aligned}
& \Gamma^{a}=\left(\begin{array}{cc}
0 & \sigma^{a} \\
\bar{\sigma}^{a} & 0
\end{array}\right), \quad \Gamma^{a b}=2\left(\begin{array}{cc}
\sigma^{a b} & 0 \\
0 & \bar{\sigma}^{a b}
\end{array}\right), \quad \Gamma^{a b c}=i \epsilon^{a b c d}\left(\begin{array}{cc}
0 & \sigma_{d} \\
-\bar{\sigma}_{d} & 0
\end{array}\right) \\
& \Gamma^{\hat{5}}=\left(\begin{array}{cc}
-i & 0 \\
0 & i
\end{array}\right), \quad \Gamma^{a \hat{5}}=i\left(\begin{array}{cc}
0 & \sigma^{a} \\
-\bar{\sigma}^{a} & 0
\end{array}\right), \quad \Gamma^{a b \hat{5}}=2 i\left(\begin{array}{cc}
-\sigma^{a b} & 0 \\
0 & \bar{\sigma}^{a b}
\end{array}\right) .
\end{aligned}
$$


A four-component Dirac spinor $\Psi$, its Dirac conjugate $\bar{\Psi}$ and its Majorana conjugate $\widetilde{\Psi}$ are written in terms of two-component spinors $\psi_{1}$ and $\psi_{2}$ as follows,

$$
\Psi=\left(\frac{\psi_{1}}{\bar{\psi}_{2}}\right), \quad \bar{\Psi}=\left(\psi_{2}, \bar{\psi}_{1}\right), \quad \widetilde{\Psi}=\left(-\psi_{1}, \bar{\psi}_{2}\right)
$$

A symplectic Majorana spinor $\Psi_{i}$ satisfies $\widetilde{\Psi}^{i}=\bar{\Psi}_{i}$, where index $i$ can be raised and lowered with an antisymmetric tensor $\varepsilon_{i j}$. We use the following representation,

$$
\Psi_{1}=-\Psi^{2}=\left(\frac{\psi_{1}}{\bar{\psi}_{2}}\right), \quad \Psi_{2}=\Psi^{1}=\left(\begin{array}{c}
-\psi_{2} \\
\bar{\psi}_{1}
\end{array}\right) .
$$

The following identities are satisfied,

$$
\widetilde{\Psi} \vec{\Gamma} \mathcal{H}=\widetilde{\mathcal{H}} \overleftarrow{\Gamma} \Psi, \quad \widetilde{\Psi}^{i} \vec{\Gamma}^{\mathcal{H}} \mathcal{H}_{i}=-\widetilde{\mathcal{H}}^{i} \stackrel{\leftarrow}{\Gamma} \Psi_{i}, \quad Q_{i}{ }^{j} \widetilde{\Psi}^{i} \vec{\Gamma} \mathcal{H}_{j}=Q_{i}{ }^{j} \widetilde{\mathcal{H}}^{i} \stackrel{\leftarrow}{\Gamma} \Psi_{j}
$$

where $\vec{\Gamma}=\Gamma^{A_{1}} \Gamma^{A_{2}} \ldots \Gamma^{A_{n}}, \overleftarrow{\Gamma}=\Gamma^{A_{n}} \ldots \Gamma^{A_{2}} \Gamma^{A_{1}}$ and

$$
Q_{i}{ }^{j}=i \vec{q} \cdot \vec{\sigma}=i\left(\begin{array}{cc}
q_{3} & q_{1}-i q_{2} \\
q_{1}+i q_{2} & -q_{3}
\end{array}\right) .
$$

Also, for arbitrary symplectic Majorana spinors we have

$$
\begin{aligned}
& i \widetilde{\Psi}^{i} \vec{\Gamma} \mathcal{H}_{i}=i \bar{\Psi}_{1} \vec{\Gamma} \mathcal{H}_{1}+\text { h.c. } \\
& i \widetilde{\Psi}^{i} \vec{\Gamma} Q_{i}{ }^{j} \mathcal{H}_{j}=-q_{3} \bar{\Psi}_{1} \vec{\Gamma} \mathcal{H}_{1}-q_{12} \bar{\Psi}_{2} \vec{\Gamma} \mathcal{H}_{1}+\text { h.c. }
\end{aligned}
$$

where $q_{12}=q_{1}+i q_{2}$. These identities allow a straightforward reduction of the action and supersymmetry transformations to the two-component expressions. The following set of expressions is especially helpful,

$$
\begin{gathered}
\bar{\Psi} \mathcal{H}=\psi_{2} \eta_{1}+\bar{\psi}_{2} \bar{\eta}_{2}, \quad \bar{\Psi} \Gamma^{\hat{5}} \mathcal{H}=-i\left(\psi_{2} \eta_{1}-\bar{\psi}_{1} \bar{\eta}_{2}\right) \\
\bar{\Psi} \Gamma^{a} \mathcal{H}=\psi_{2} \sigma^{a} \bar{\eta}_{2}+\bar{\psi}_{1} \bar{\sigma}^{a} \eta_{1}, \quad \bar{\Psi} \Gamma^{a \hat{5}} \mathcal{H}=i\left(\psi_{2} \sigma^{a} \bar{\eta}_{2}-\bar{\psi}_{1} \bar{\sigma}^{a} \eta_{1}\right) \\
\bar{\Psi} \Gamma^{a b} \mathcal{H}=2\left(\psi_{2} \sigma^{a b} \eta_{1}+\bar{\psi}_{1} \bar{\sigma}^{a b} \bar{\eta}_{2}\right), \quad \bar{\Psi} \Gamma^{a b \hat{5}} \mathcal{H}=-2 i\left(\psi_{2} \sigma^{a b} \eta_{1}-\bar{\psi}_{1} \bar{\sigma}^{a b} \bar{\eta}_{2}\right) .
\end{gathered}
$$

Finally,

$$
\bar{\Psi} \Gamma^{a b c d} \mathcal{H}=\epsilon^{a b c d} \bar{\Psi} \Gamma^{\hat{5}} \mathcal{H}, \quad \bar{\Psi} \Gamma^{a b c \hat{5}} \mathcal{H}=-\epsilon^{a b c d} \bar{\Psi} \Gamma_{d} \mathcal{H}
$$




\section{B. Gibbons-Hawking boundary term}

We define the extrinsic curvature as ${ }^{13}$

$$
K_{M N}=P_{M}^{K} P_{N}{ }^{L} D_{K} n_{L},
$$

where $n^{M}$ is the (outward pointing) unit vector normal to the boundary $\partial \mathcal{M}$, and

$$
P_{M}^{N}=\delta_{M}^{N}-n_{M} n^{N}
$$

is a projector onto the boundary, $P_{M}{ }^{N} n_{N}=0$. The trace of the extrinsic curvature is

$$
K=g^{M N} K_{M N}
$$

One can show [15] that its general variation gives

$$
\delta K=K_{M N} e_{A}^{M} \delta e^{N A}+n_{M}\left(e^{M A} e^{N B} \delta \omega_{N A B}\right)+P_{M}^{K} D_{K}\left(e_{A}^{L} P_{L}^{M} n_{N} \delta e^{N A}\right) .
$$

The last term is a total tangential derivative which vanishes upon integrating over $\partial \mathcal{M}$. Since

$$
\delta \int_{\mathcal{M}}\left(-\frac{1}{2} R\right)=\int_{\mathcal{M}}\left(R_{A}^{M}-\frac{1}{2} R e_{A}^{M}\right) \delta e_{M}^{A}+\int_{\partial \mathcal{M}}\left(-n_{M} e^{M A} e^{N B} \delta \omega_{N A B}\right)
$$

we see that the Einstein-Hilbert action with the Gibbons-Hawking boundary term,

$$
S_{E H+G H}=-\frac{1}{2} \int_{\mathcal{M}} R+\int_{\partial \mathcal{M}} K
$$

under the general variation gives

$$
\delta S_{E H+G H}=\int_{\mathcal{M}}\left(R_{A}^{M}-\frac{1}{2} R e_{A}^{M}\right) \delta e_{M}^{A}+\int_{\partial \mathcal{M}}\left(K_{M N}-K P_{M N}\right) e_{A}^{N} \delta e^{M A}
$$

The Gibbons-Hawking term makes only the variation of the metric (vielbein $e^{M A}$ ) appear in the boundary term of the general variation of the total action. This improves the variational principle, allowing both the use of the Dirichlet boundary conditions for the metric,

$$
\delta e_{M}^{A}=0 \text { on } \partial \mathcal{M}
$$

and the derivation of the "natural" (generalized Neumann) boundary conditions,

$$
K_{M N}-K P_{M N}=S_{M N} \quad \text { on } \partial \mathcal{M}
$$

where $S_{M N}$ represents a contribution from a boundary action.

\footnotetext{
${ }^{13}$ For a detailed discussion of the extrinsic curvature see Refs. [38, 39, 40].
} 


\section{Why we choose $n_{5}=-e_{5}^{\hat{5}}$}

The Stokes's theorem states (see, e.g., Ref. [40])

$$
\int_{\mathcal{M}} d^{5} x e_{5}\left(D_{M} K^{M}\right)=\int_{\mathcal{M}} d^{5} x \partial_{M}\left(e_{5} K^{M}\right)=\int_{\partial \mathcal{M}} d^{4} x e_{4}^{\mathrm{ind}}\left(n_{M} K^{M}\right)
$$

where (denoting by $g_{m n}^{\text {ind }}$ the induced four-dimensional metric on $\partial \mathcal{M}$ )

$$
\begin{aligned}
e_{5} & =\operatorname{det} e_{M A}=\sqrt{\left|\operatorname{det} g_{M N}\right|} \\
e_{4}^{\text {ind }} & =\operatorname{det} e_{m a}^{\text {ind }}=\sqrt{\left|\operatorname{det} g_{m n}^{\text {ind }}\right|},
\end{aligned}
$$

and $n_{M} 1$ ) is orthogonal to $\partial \mathcal{M} ; 2$ ) has the unit norm, $\left.g^{M N} n_{M} n_{N}=1 ; 3\right)$ is outward pointing. With our description of $\partial \mathcal{M}$ as a hypersurface $x^{5}=$ const, the first condition implies that only $n_{5} \neq 0$, the second says

$$
n_{5}= \pm \frac{1}{\sqrt{g^{55}}}
$$

and the third has to do with choosing one of the two signs.

In our gauge $\left(e_{m}^{\hat{5}}=0\right)$, we have $g^{55}=e_{\hat{5}}^{5} e_{\hat{5}}^{5}$ and $e_{5}^{\hat{5}} e_{\hat{5}}^{5}=1$, thus

$$
n_{5}= \pm e_{5}^{\hat{5}}
$$

In this gauge we also have $g_{m n}^{\text {ind }}=e_{m}^{a} e_{n a}$ and, therefore, we can choose the induced vierbein as $e_{m a}^{\text {ind }}=e_{m a}$ and obtain $e_{5}=e_{4} e_{5}^{\hat{5}}$.

Let us assume that our $\mathcal{M}$ is a strip $x^{5} \equiv z \in\left[z_{1}, z_{2}\right]$ and $\Sigma$ denotes an $x^{5}=$ const hypersurface. The Stokes's theorem can then be written as

$$
\int_{\Sigma} d^{4} x \int_{z_{1}}^{z_{2}} d z \partial_{5}\left(e_{4} e_{5}^{\hat{5}} K^{5}\right)=\int_{\partial \mathcal{M}} d^{4} x e_{4}\left(n_{5} K^{5}\right),
$$

and, therefore,

$$
\left(e_{5}^{\hat{5}} K^{5}\right)_{\mid z_{2}}-\left(e_{5}^{\hat{5}} K^{5}\right)_{\mid z_{1}}=\left(n_{5} K^{5}\right)_{\mid z_{2}}+\left(n_{5} K^{5}\right)_{\mid z_{1}} .
$$

This means that for the Stokes's theorem to hold (that is for $n_{M}$ to be "outward pointing"), we should choose

$$
n_{5}=-e_{5}^{\hat{5}} \text { at } z_{1} \quad \text { and } \quad n_{5}=+e_{5}^{\hat{5}} \text { at } z_{2} .
$$

This choice coincides with the intuitive one when $e_{5}^{\hat{5}}>0$.

When $\mathcal{M}=\mathcal{M}_{+}=\mathbb{R}^{1,3} \times[0,+\infty)$, the outward pointing $n_{M}$ at $z=0$ has $n_{5}=-e_{5}^{\hat{5}}$. With this choice, the boundary conditions we obtain on $\partial \mathcal{M}$ coincide with the boundary conditions "on the positive side of the brane" (that is "at $z=+0$ ") and thus directly correspond to the boundary conditions in Refs. [7] and [8]. 


\section{Our gauge}

Our gauge choice is $e_{m}^{\hat{5}}=0$. Thus,

$$
e_{m}^{\hat{5}}=0, \quad e_{a}^{5}=0, \quad e_{5}^{a} \neq 0, \quad e_{\hat{5}}^{m} \neq 0 .
$$

Since $e_{A}^{M}$ is the inverse to $e_{M}^{A}$,

$$
e_{M}^{A} e_{A}^{N}=\delta_{M}^{N}, \quad e_{A}^{M} e_{M}^{B}=\delta_{A}^{B},
$$

in this gauge we have

$$
e_{m}^{a} e_{a}^{n}=\delta_{m}^{n}, \quad e_{a}^{m} e_{m}^{b}=\delta_{a}^{b}, \quad e_{5}^{\hat{5}} e_{\hat{5}}^{5}=1, \quad e_{\hat{5}}^{m}=-e_{5}^{a} e_{a}^{m} e_{\hat{5}}^{5} .
$$

\section{D.1 Metric tensor}

For the metric tensor $g_{M N}$ we obtain

$$
g_{m n}=\gamma_{m n}, \quad g_{m 5}=g_{5 m}=N_{m}, \quad g_{55}=\gamma^{m n} N_{m} N_{n}+N^{2},
$$

where we defined

$$
\gamma_{m n} \equiv e_{m}^{a} e_{n a}, \quad N_{m} \equiv e_{5}^{a} e_{m a}, \quad N=e_{5}^{\hat{5}},
$$

and $\gamma^{m n}$ is the inverse to $\gamma_{m n}$,

$$
\gamma^{m n} \equiv e^{m a} e_{a}^{n}, \quad \gamma_{m k} \gamma^{k n}=\delta_{m}^{n} .
$$

Defining

$$
N^{m} \equiv \gamma^{m n} N_{n}=e_{5}^{a} e_{a}^{m}=-N e_{\hat{5}}^{m},
$$

the inverse five dimensional metric tensor $g^{M N}$ can be written as

$$
g^{m n}=\gamma^{m n}+N^{-2} N^{m} N^{n}, \quad g^{m 5}=g^{5 m}=-N^{-2} N^{m}, \quad g^{55}=N^{-2} .
$$

\section{D.2 Field strength}

Components of $F^{M N}=g^{M K} g^{N L} F_{K L}$ for the field strength $F_{M N}=\partial_{M} B_{N}-\partial_{N} B_{M}$ are

$$
\begin{aligned}
& F^{m n}=g^{m k} g^{n l} F_{k l}+\left(g^{m k} g^{n 5}-g^{n k} g^{m 5}\right) F_{m 5} \\
& F^{m 5}=g^{m n} g^{k 5} F_{n k}+\left(g^{m n} g^{55}-g^{m 5} g^{n 5}\right) F_{n 5} .
\end{aligned}
$$

We can write this in a more convenient form,

$$
F^{m n}=\gamma^{m k} \gamma^{n l} F_{k l}+\gamma^{m k} e_{\hat{5}}^{n} F_{k \hat{5}}-\gamma^{n k} e_{\hat{5}}^{m} F_{k \hat{5}}, \quad F^{m 5}=\gamma^{m n} e_{\hat{5}}^{5} F_{n \hat{5}},
$$

where we defined

$$
F_{m \hat{5}} \equiv e_{\hat{5}}^{5} F_{m 5}+e_{\hat{5}}^{n} F_{m n}
$$




\section{D.3 Spin connection}

We use the following spin connection,

$$
\omega_{M A B}=e_{A}^{N} e_{B}^{K} \omega_{M N K}, \quad \omega_{M N K}=\frac{1}{2}\left(C_{M N K}+C_{N M K}-C_{K M N}\right),
$$

where

$$
C_{M N K}=e_{M C}\left(\partial_{N} e_{K}^{C}-\partial_{K} e_{N}^{C}\right)
$$

We find that in our gauge

$$
\begin{aligned}
& C_{m n k}=\widehat{C}_{m n k}, \quad C_{m n 5}=u_{m n} \\
& C_{5 m n}=N^{k} \widehat{C}_{k m n}, \quad C_{5 n 5}=N^{k} u_{k n}+N \partial_{n} N,
\end{aligned}
$$

where

$$
\widehat{C}_{m n k} \equiv e_{m c}\left(\partial_{n} e_{k}^{c}-\partial_{k} e_{n}^{c}\right), \quad u_{m n} \equiv e_{m c}\left(\partial_{n} e_{5}^{c}-\partial_{5} e_{n}^{c}\right)
$$

The spin connection coefficients are given by

$$
\begin{aligned}
\omega_{m a b} & =\frac{1}{2} e_{a}^{n} e_{b}^{k}\left(\widehat{C}_{m n k}+\widehat{C}_{n m k}-\widehat{C}_{k m n}\right) \\
\omega_{5 a b} & =\frac{1}{2} N^{n} \widehat{C}_{n a b}-\frac{1}{2}\left(u_{a b}-u_{b a}\right) \\
\omega_{m a \hat{5}} & =-\frac{1}{2} N^{-1} N^{k}\left(\widehat{C}_{m a k}+\widehat{C}_{a m k}\right)+\frac{1}{2} N^{-1}\left(u_{a m}+u_{m a}\right) \\
\omega_{5 a \hat{5}} & =-\frac{1}{2} N^{-1} N^{n} N^{k} \widehat{C}_{n a k}+\frac{1}{2} N^{-1} N^{k}\left(u_{k a}+u_{a k}\right)+e_{a}^{n} \partial_{n} N .
\end{aligned}
$$

We see that in our gauge there are no $\partial_{5} N_{m}$ in any of $\omega_{M A B}$, which means that the spin connection coefficients are non-singular, i.e. contain no $\delta(z)$, in the orbifold picture!

\section{D.4 Extrinsic curvature}

Our (outward pointing) unit vector normal to the boundary $\partial \mathcal{M}$ is

$$
n_{M}=\left(0_{m},-N\right), \quad n^{M}=\left(N^{-1} N^{m},-N^{-1}\right) .
$$

This gives the following projector onto the boundary,

$$
P^{M N}=\left(\begin{array}{cc}
\gamma^{m n} & 0 \\
0 & 0
\end{array}\right) \quad P_{M}^{N}=\left(\begin{array}{cc}
\delta_{m}^{n} & 0 \\
N^{n} & 0
\end{array}\right) \quad P_{M N}=\left(\begin{array}{cc}
\gamma_{m n} & N_{m} \\
N_{n} & N^{k} N_{k}
\end{array}\right)
$$

and the extrinsic curvature,

$$
K^{M N}=\left(\begin{array}{cc}
K^{m n} & 0 \\
0 & 0
\end{array}\right) \quad K_{M N}=\left(\begin{array}{cc}
K_{m n} & N^{k} K_{m k} \\
N^{k} K_{k n} & N^{k} N^{l} K_{k l}
\end{array}\right),
$$


where

$$
K_{m n}=-\Gamma_{m n}^{5} n_{5}, \quad K^{m n}=\gamma^{m k} \gamma^{n l} K_{k l} .
$$

The trace of the extrinsic curvature is

$$
K \equiv g_{M N} K^{M N}=\gamma_{m n} K^{m n}=\gamma^{m n} K_{m n}=-\gamma^{m n} \Gamma_{m n}^{5} n_{5} .
$$

Using the relation between the Christoffel symbols and the spin connection,

$$
D_{M} e_{N}^{A} \equiv \partial_{M} e_{N}^{A}+\omega_{M C}^{A} e_{N}^{C}-\Gamma_{M N}^{K} e_{K}^{A}=0,
$$

we find

$$
\begin{aligned}
\Gamma_{m n}^{5}=\omega_{m n}{ }^{5}+e_{A}^{5} \partial_{m} e_{n}^{A}= & e_{n}^{a} \omega_{m a}{ }^{\hat{5}} e_{\hat{5}}^{5} \\
& +e_{n}^{a} \omega_{m a}^{b} e_{b}^{5}+e_{n}^{\hat{5}} \omega_{m \hat{5}^{b}} e_{b}^{5}+e_{a}^{5} \partial_{m} e_{n}^{a}+e_{\hat{5}}^{5} \partial_{m} e_{n}^{\hat{5}}
\end{aligned}
$$

But all the terms in the second line vanish in our gauge, so

$$
\Gamma_{m n}^{5}=e_{n}^{a} \omega_{m a} \hat{s}_{\hat{5}}^{5}
$$

Therefore, we obtain

$$
K_{m a} \equiv e_{a}^{n} K_{m n}=\omega_{m a \hat{5}}, \quad K=e^{m a} K_{m a} .
$$

This gives a geometrical meaning to the spin connection coefficient $\omega_{m a \hat{5}}$.

\section{D.5 Advantages of the $e_{m}^{\hat{5}}=0$ gauge}

The following properties are unique to our gauge, $e_{m}^{\hat{5}}=0$. (Another simple gauge, $e_{5}^{a}=0$, frequently used in the Kaluza-Klein reductions, ${ }^{14}$ does not enjoy these properties.)

1. $e_{m}^{a}$ is an induced vierbein on a slice $x^{5}=$ const.

2. $\omega_{m a b}$ is a spin connection for $e_{m}^{a}$.

3. $\widehat{D}_{m} e_{n}^{a}=\partial_{m} e_{n}^{a}+\omega_{m c}{ }^{a} e_{n}^{c}-\Gamma_{m n}^{k} e_{k}^{a}=0$, where $\omega_{m a b}$ and $\Gamma_{m n}^{k}$ are elements of $\omega_{M A B}$ and $\Gamma_{M N}^{K} \cdot$

4. There is a simple relation between the extrinsic curvature and a spin connection coefficient: $K_{m a}=\omega_{m a \hat{5}}$.

5. There are no $\delta(z)$-terms in any of the spin connection coefficients $\omega_{M A B}$.

6. The compensating local Lorentz rotation (with the parameter $\omega^{a \hat{5}}$; see below) leaves the supersymmetry transformation of $e_{m}^{a}$ unchanged.

\footnotetext{
${ }^{14}$ See, e.g., the paper by Chamseddine and Nicolai in Ref. [19].
} 


\section{E. Supersymmetry transformations}

In our gauge and in the two-component spinor notation, the supersymmetry transformations of Eqs. 2.1 2.3) (dropping the 3-Fermi terms in the $\delta \Psi_{M i}$ ) can be written as follows,

$$
\begin{aligned}
& \delta_{\mathcal{H}} e_{m}^{a}=-i\left(\psi_{m 1} \sigma^{a} \bar{\eta}_{1}+\psi_{m 2} \sigma^{a} \bar{\eta}_{2}\right)+\text { h.c. } \\
& \delta_{\mathcal{H}} e_{5}^{a}=-i\left(\psi_{51} \sigma^{a} \bar{\eta}_{1}+\psi_{52} \sigma^{a} \bar{\eta}_{2}\right)+\text { h.c. } \\
& \delta_{\mathcal{H}} e_{m}^{\hat{5}}=-\psi_{m 2} \eta_{1}+\psi_{m 1} \eta_{2}+\text { h.c. } \\
& \delta_{\mathcal{H}} e_{5}^{\hat{5}}=-\psi_{52} \eta_{1}+\psi_{51} \eta_{2}+\text { h.c. } \\
& \delta_{\mathcal{H}} B_{m}=i \frac{\sqrt{6}}{2}\left(\psi_{m 2} \eta_{1}-\psi_{m 1} \eta_{2}\right)+\text { h.c. } \\
& \delta_{\mathcal{H}} B_{5}=i \frac{\sqrt{6}}{2}\left(\psi_{52} \eta_{1}-\psi_{51} \eta_{2}\right)+\text { h.c. } \\
& \delta_{\mathcal{H}} \psi_{m 1}=2 \widehat{D}_{m} \eta_{1}+i \omega_{m a \hat{5}} \sigma^{a} \bar{\eta}_{2}+i \lambda \sigma_{m}\left(q_{12}^{*} \bar{\eta}_{1}+q_{3} \bar{\eta}_{2}\right)-i \sqrt{6} \lambda\left(q_{3} \eta_{1}-q_{12}^{*} \eta_{2}\right) B_{m} \\
& +\frac{1}{2 \sqrt{6}}\left\{-4 i\left(\sigma_{m}^{n}+\delta_{m}^{n}\right) \eta_{1}\left(e_{\hat{5}}^{5} F_{n 5}+e_{\hat{5}}^{k} F_{n k}\right)+\left[i \epsilon_{m}^{n k l} \sigma_{l}+4 \delta_{m}^{n} \sigma^{k}\right] \bar{\eta}_{2} F_{n k}\right\} \\
& \delta_{\mathcal{H}} \psi_{m 2}=2 \widehat{D}_{m} \eta_{2}-i \omega_{m a \hat{5}} \sigma^{a} \bar{\eta}_{1}+i \lambda \sigma_{m}\left(q_{3} \bar{\eta}_{1}-q_{12} \bar{\eta}_{2}\right)+i \sqrt{6} \lambda\left(q_{3} \eta_{2}+q_{12} \eta_{1}\right) B_{m} \\
& +\frac{1}{2 \sqrt{6}}\left\{-4 i\left(\sigma_{m}^{n}+\delta_{m}^{n}\right) \eta_{2}\left(e_{\tilde{5}}^{5} F_{n 5}+e_{\tilde{5}}^{k} F_{n k}\right)-\left[i \epsilon_{m}{ }^{n k l} \sigma_{l}+4 \delta_{m}^{n} \sigma^{k}\right] \bar{\eta}_{1} F_{n k}\right\} \\
& \delta_{\mathcal{H}} \psi_{51}=2 \widehat{D}_{5} \eta_{1}+i \omega_{5 a \hat{5}} \sigma^{a} \bar{\eta}_{2}+\lambda\left(e_{5}^{\hat{5}}-i \sqrt{6} B_{5}\right)\left(q_{3} \eta_{1}-q_{12}^{*} \eta_{2}\right) \\
& +\frac{1}{2 \sqrt{6}}\left\{-4\left(\sigma^{n} \bar{\eta}_{2}-i e_{\tilde{5}}^{n} \eta_{1}\right) F_{n 5}-4 i e_{5 a} \sigma^{a n} \eta_{1}\left(e_{\tilde{5}}^{5} F_{n 5}+e_{\tilde{5}}^{k} F_{n k}\right)\right. \\
& \left.+\left(-2 i e_{5}^{\hat{5}} \sigma^{n k} \eta_{1}+i e_{5 a} \epsilon^{a n k l} \sigma_{l} \bar{\eta}_{2}\right) F_{n k}\right\} \\
& \delta_{\mathcal{H}} \psi_{52}=2 \widehat{D}_{5} \eta_{2}-i \omega_{5 a \hat{5}} \sigma^{a} \bar{\eta}_{1}-\lambda\left(e_{5}^{\hat{5}}-i \sqrt{6} B_{5}\right)\left(q_{3} \eta_{2}+q_{12} \eta_{1}\right) \\
& +\frac{1}{2 \sqrt{6}}\left\{+4\left(\sigma^{n} \bar{\eta}_{1}+i e_{\hat{5}}^{n} \eta_{2}\right) F_{n 5}-4 i e_{5 a} \sigma^{a n} \eta_{2}\left(e_{\hat{5}}^{5} F_{n 5}+e_{\hat{5}}^{k} F_{n k}\right)\right. \\
& \left.+\left(-2 i e_{5}^{\hat{5}} \sigma^{n k} \eta_{2}-i e_{5 a} \epsilon^{a n k l} \sigma_{l} \bar{\eta}_{1}\right) F_{n k}\right\}
\end{aligned}
$$

where

$$
\widehat{D}_{M} \eta=\partial_{M} \eta+\frac{1}{2} \omega_{M a b} \sigma^{a b} \eta
$$

Note that all $e_{5}^{a}$ and $e_{\tilde{5}}^{m}$ have been explicitly separated out (thus, $\sigma^{m}=e_{a}^{m} \sigma^{a}$ and so on).

However, we have to modify the supersymmetry transformations by a compensating Lorentz transformation $\left(\omega_{A B}=-\omega_{B A}\right)$,

$$
\delta_{\omega} e_{M}^{A}=e_{M}^{B} \omega_{B}^{A}, \quad \delta_{\omega} \Psi_{M i}=\frac{1}{4} \omega_{A B} \Gamma^{A B} \Psi_{M i}
$$


in order to stay in our $e_{m}^{\hat{5}}=0$ gauge. Using only off-diagonal coefficient $\omega^{a \hat{5}}$,

$$
\delta_{\omega} e_{m}^{a}=-e_{m \hat{5}} \omega^{a \hat{5}}, \quad \delta_{\omega} e_{5}^{a}=-e_{5 \hat{5}} \omega^{a \hat{5}}, \quad \delta_{\omega} e_{m}^{\hat{5}}=e_{m c} \omega^{c \hat{5}}, \quad \delta_{\omega} e_{5}^{\hat{5}}=e_{5 c} \omega^{c \hat{5}}
$$

We define the modified supersymmetry transformations by

$$
\delta_{\mathcal{H}}^{\prime} e_{M}^{A}=\delta_{\mathcal{H}} e_{M}^{A}+\delta_{\omega} e_{M}^{A}
$$

We want the supersymmetry variation to preserve $e_{m}^{\hat{5}}=0$,

$$
\delta_{\mathcal{H}}^{\prime} e_{m}^{\hat{5}}=\delta_{\mathcal{H}} e_{m}^{\hat{5}}+e_{m c} \omega^{c \hat{5}}=0
$$

which fixes

$$
\omega^{a \hat{5}}=-e^{m a} \delta_{\mathcal{H}} e_{m}^{\hat{5}}=e^{m a}\left(\psi_{m 2} \eta_{1}-\psi_{m 1} \eta_{2}\right)+\text { h.c. }
$$

The modified supersymmetry transformations, therefore, are

$$
\begin{aligned}
\delta_{\mathcal{H}}^{\prime} e_{m}^{a} & =\delta_{\mathcal{H}} e_{m}^{a} \\
\delta_{\mathcal{H}}^{\prime} e_{5}^{a} & =\delta_{\mathcal{H}} e_{5}^{a}+e_{5 \hat{5}} e^{m a} \delta_{\mathcal{H}} e_{m}^{\hat{5}} \\
\delta_{\mathcal{H}}^{\prime} e_{m}^{\hat{5}} & =0 \\
\delta_{\mathcal{H}}^{\prime} e_{5}^{\hat{5}} & =\delta_{\mathcal{H}} e_{5}^{\hat{5}}-e_{5}^{a} e_{a}^{m} \delta_{\mathcal{H}} e_{m}^{\hat{5}} .
\end{aligned}
$$

Note that the supersymmetry variation of the $e_{m}^{a}$ stays the same, which is one of the advantages of our gauge.

The gravitino supersymmetry transformations also get modified, but only in the 3-Fermi terms (since $\omega^{a \hat{5}}$ is 2 -Fermi) which we omit.

\section{F. Bulk Lagrangian}

The fermionic part of the bulk supergravity Lagrangian is

$$
\begin{aligned}
\mathcal{L}_{5 F}= & \frac{i}{2} \widetilde{\Psi}_{M}^{i} \Gamma^{M N K} D_{N} \Psi_{K i}-i \frac{\sqrt{6}}{8} F^{M N} \widetilde{\Psi}_{M}^{i} \Psi_{N i}-i \frac{\sqrt{6}}{16} F_{M N} \widetilde{\Psi}_{P}^{i} \Gamma^{M N P Q} \Psi_{Q i} \\
& +i \frac{3}{4} \lambda Q_{i}{ }^{j} \widetilde{\Psi}_{M}^{i} \Gamma^{M N} \Psi_{N j}-i \frac{\sqrt{6}}{4} \lambda Q_{i}{ }^{j} \widetilde{\Psi}_{M}^{i} \Gamma^{M N K} \Psi_{K j} B_{N}
\end{aligned}
$$


In the two-component spinor notation it becomes

$$
\begin{aligned}
& \mathcal{L}_{5 F}=\frac{1}{2} \epsilon^{m n k l}\left(\bar{\psi}_{m 2} \bar{\sigma}_{l} D_{n} \psi_{k 2}+\bar{\psi}_{m 1} \bar{\sigma}_{l} D_{n} \psi_{k 1}\right)+\left(\psi_{m 1} \sigma^{m n} D_{\hat{5}} \psi_{n 2}-\psi_{m 2} \sigma^{m n} D_{\hat{5}} \psi_{n 1}\right) \\
& +\left(\psi_{m 2} \sigma^{m n} D_{n} \psi_{\hat{5} 1}-\psi_{\hat{5} 1} \sigma^{m n} D_{m} \psi_{n 2}\right)+\left(\psi_{\hat{5} 2} \sigma^{m n} D_{m} \psi_{n 1}-\psi_{m 1} \sigma^{m n} D_{n} \psi_{\hat{5} 2}\right) \\
& -i \frac{\sqrt{6}}{4}\left[\gamma^{m k} \gamma^{n l} F_{k l}\left(\psi_{m 2} \psi_{n 1}\right)+\gamma^{m k} F_{k \hat{5}}\left(\psi_{m 2} \psi_{\hat{5} 1}-\psi_{m 1} \psi_{\hat{5} 2}\right)\right] \\
& -\frac{\sqrt{6}}{8} \epsilon^{m n p q}\left[F_{p q}\left(\psi_{m 2} \psi_{n 1}\right)-i F_{p q}\left(\psi_{m 2} \sigma_{n} \bar{\psi}_{\hat{5} 2}+\psi_{m 1} \sigma_{n} \bar{\psi}_{\hat{5} 1}\right)\right. \\
& \left.-i F_{m \hat{5}}\left(\psi_{p 2} \sigma_{n} \bar{\psi}_{q 2}+\psi_{p 1} \sigma_{n} \bar{\psi}_{q 1}\right)\right] \\
& -\frac{3}{2} \lambda\left\{q_{3}\left[2 \psi_{m 1} \sigma^{m n} \psi_{n 2}+i\left(\psi_{m 2} \sigma^{m} \bar{\psi}_{\hat{5}_{2}}-\psi_{m 1} \sigma^{m} \bar{\psi}_{\hat{5} 1}\right)\right]\right. \\
& \left.+q_{12}\left[\left(\psi_{m 1} \sigma^{m n} \psi_{n 1}-\bar{\psi}_{m 2} \bar{\sigma}^{m n} \bar{\psi}_{n 2}\right)+i\left(\psi_{m 1} \sigma^{m} \bar{\psi}_{\hat{5}_{2}}+\bar{\psi}_{m 2} \bar{\sigma}^{m} \psi_{\hat{5} 1}\right)\right]\right\} \\
& +\frac{\sqrt{6}}{4} \lambda\left\{q _ { 3 } \left[i B_{n} \epsilon^{m n k l}\left(\psi_{m 2} \sigma_{l} \bar{\psi}_{k 2}-\psi_{m 1} \sigma_{l} \bar{\psi}_{k 1}\right)+4 i B_{\hat{5}}\left(\psi_{m 1} \sigma^{m n} \psi_{n 2}\right)\right.\right. \\
& \left.-4 i B_{n}\left(\psi_{m 2} \sigma^{m n} \psi_{\hat{5} 1}+\psi_{m 1} \sigma^{m n} \psi_{\hat{5} 2}\right)\right] \\
& +q_{12}\left[2 i B_{n} \epsilon^{m n k l}\left(\psi_{m 1} \sigma_{l} \bar{\psi}_{k 2}\right)+2 i B_{\hat{5}}\left(\psi_{m 1} \sigma^{m n} \psi_{n 1}+\bar{\psi}_{m 2} \bar{\sigma}^{m n} \bar{\psi}_{n 2}\right)\right. \\
& \left.\left.-4 i B_{n}\left(\psi_{m 1} \sigma^{m n} \psi_{\hat{5} 1}+\bar{\psi}_{m 2} \bar{\sigma}^{m n} \bar{\psi}_{\hat{5}_{2}}\right)\right]\right\} \\
& \text { +h.c. }
\end{aligned}
$$

Note that $e_{5}^{a}$ does not appear at all, whereas $e_{\tilde{5}}^{m}$ appears only in the following combinations,

$$
\begin{array}{ll}
B_{\hat{5}}=e_{\hat{5}}^{5} B_{5}+e_{\hat{5}}^{m} B_{m}, & F_{m \hat{5}}=e_{\hat{5}}^{5} F_{m 5}+e_{\hat{5}}^{n} F_{m n} \\
D_{\hat{5}}=e_{\hat{5}}^{5} D_{5}+e_{\hat{5}}^{m} D_{m}, & \psi_{\hat{5} 1,2}=e_{\hat{5}}^{5} \psi_{51,2}+e_{\hat{5}}^{m} \psi_{m 1,2} .
\end{array}
$$

In particular, $\sigma^{m}=e_{a}^{m} \sigma^{a}$ and $\epsilon^{m n k l}=e_{a}^{m} e_{b}^{n} e_{c}^{k} e_{d}^{l} \epsilon^{a b c d}$. The derivatives can be further decomposed as follows,

$$
\begin{aligned}
D_{M} \psi_{1} & =\widehat{D}_{M} \psi_{1}+\frac{i}{2} \omega_{M a \hat{5}} \bar{\psi}_{2} \\
D_{M} \psi_{2} & =\widehat{D}_{M} \psi_{2}-\frac{i}{2} \omega_{M a \hat{5}} \bar{\psi}_{1}
\end{aligned}
$$

Note that $D_{M} e_{N}^{A}=0$, but $\widehat{D}_{M} e_{N}^{A} \neq 0$.

\section{References}

[1] P. Horava and E. Witten, "Eleven-Dimensional Supergravity on a Manifold with Boundary," Nucl. Phys. B 475, 94 (1996) [arXiv:hep-th/9603142]. 
[2] L. Randall and R. Sundrum, "A large mass hierarchy from a small extra dimension," Phys. Rev. Lett. 83, 3370 (1999) [arXiv:hep-ph/9905221].

[3] L. Randall and R. Sundrum, "An alternative to compactification," Phys. Rev. Lett. 83, 4690 (1999) [arXiv:hep-th/9906064].

[4] R. Altendorfer, J. Bagger and D. Nemeschansky, "Supersymmetric Randall-Sundrum scenario," Phys. Rev. D 63, 125025 (2001) [hep-th/0003117].

[5] T. Gherghetta and A. Pomarol, "Bulk fields and supersymmetry in a slice of AdS," Nucl. Phys. B 586, 141 (2000) [hep-ph/0003129].

[6] A. Falkowski, Z. Lalak and S. Pokorski, "Supersymmetrizing branes with bulk in five-dimensional supergravity," Phys. Lett. B 491, 172 (2000) [hep-th/0004093].

[7] J. Bagger and D. V. Belyaev, "Supersymmetric branes with (almost) arbitrary tensions," Phys. Rev. D 67, 025004 (2003) [arXiv:hep-th/0206024].

[8] J. Bagger and D. Belyaev, "Twisting warped supergravity," JHEP 0306, 013 (2003) [arXiv:hep-th/0306063].

[9] G. W. Gibbons and S. W. Hawking, "Action Integrals And Partition Functions In Quantum Gravity," Phys. Rev. D 15, 2752 (1977).

[10] J. W. York, Jr.; "Role of conformal three-geometry in the dynamics of gravitation," Phys. Rev. Lett. 28, 1082 (1972).

[11] J. W. York, Jr.; "Boundary terms in the action principles of general relativity," Foundations of Physics, 16, 249 (1986).

[12] N. H. Barth, "The Fourth Order Gravitational Action For Manifolds With Boundaries," Class. Quant. Grav. 2, 497 (1985).

[13] D. V. Belyaev, "Boundary conditions in the Mirabelli and Peskin model," arXiv:hep-th/0509171.

[14] E. A. Mirabelli and M. E. Peskin, "Transmission of supersymmetry breaking from a 4-dimensional boundary," Phys. Rev. D 58, 065002 (1998) [arXiv:hep-th/9712214].

[15] D. V. Belyaev, "Five-Dimensional Supergravity on a Manifold with Boundary," Ph.D. thesis.

[16] E. Bergshoeff, R. Kallosh and A. Van Proeyen, "Supersymmetry in singular spaces," JHEP 0010, 033 (2000) [arXiv:hep-th/0007044].

[17] P. Van Nieuwenhuizen, "Supergravity," Phys. Rept. 68, 189 (1981).

[18] M. Zucker, "Off-shell supergravity in five-dimensions and supersymmetric brane world scenarios," Fortsch. Phys. 51, 899 (2003).

[19] E. Cremmer, "Supergravities In 5 Dimensions," in Superspace and supergravity, S.W. Hawking and M. Rocek eds., Cambridge University Press, 1981, pp.267-282;

A. H. Chamseddine and H. Nicolai, "Coupling The SO(2) Supergravity Through Dimensional Reduction," Phys. Lett. B 96, 89 (1980);

R. D'Auria, E. Maina, T. Regge and P. Fre, "Geometrical First Order Supergravity In Five Space-Time Dimensions," Annals Phys. 135, 237 (1981);

M. Gunaydin, G. Sierra and P. K. Townsend, "The Geometry Of N=2 Maxwell-Einstein 
Supergravity And Jordan Algebras," Nucl. Phys. B 242, 244 (1984); "Gauging The D = 5

Maxwell-Einstein Supergravity Theories: More On Jordan Algebras," Nucl. Phys. B 253, 573 (1985).

[20] P. Di Vecchia, B. Durhuus, P. Olesen and J. L. Petersen, "Fermionic Strings With Boundary Terms," Nucl. Phys. B 207, 77 (1982).

[21] P. Di Vecchia, B. Durhuus, P. Olesen and J. L. Petersen, "Fermionic Strings With Boundary Terms. 2. The O(2) String," Nucl. Phys. B 217, 395 (1983).

[22] H. Luckock and I. Moss, "The Quantum Geometry Of Random Surfaces And Spinning Membranes," Class. Quant. Grav. 6, 1993 (1989).

[23] G. von Gersdorff, L. Pilo, M. Quiros, A. Riotto and V. Sanz, "Fermions and supersymmetry breaking in the interval," Phys. Lett. B 598, 106 (2004) [arXiv:hep-th/0404091].

[24] U. Lindstrom, M. Rocek and P. van Nieuwenhuizen, "Consistent boundary conditions for open strings," Nucl. Phys. B 662, 147 (2003) [arXiv:hep-th/0211266];

P. van Nieuwenhuizen and D. V. Vassilevich, "Consistent boundary conditions for supergravity," arXiv:hep-th/0507172.

[25] J. O. Conrad, "Brane tensions and coupling constants from within M-theory," Phys. Lett. B 421, 119 (1998) [arXiv:hep-th/9708031].

[26] A. Bilal, J. P. Derendinger and R. Sauser, "M-theory on $S(1) / Z(2)$ : new facts from a careful analysis," Nucl. Phys. B 576, 347 (2000) [arXiv:hep-th/9912150].

[27] Z. Lalak and R. Matyszkiewicz, "Twisted supergravity and untwisted super-bigravity," Phys. Lett. B 562, 347 (2003) [arXiv:hep-th/0303227].

[28] G. von Gersdorff, L. Pilo, M. Quiros, A. Riotto and V. Sanz, "Supersymmetry from boundary conditions," Nucl. Phys. B 712, 3 (2005) [arXiv:hep-th/0411133].

[29] M. Zucker, "Supersymmetric brane world scenarios from off-shell supergravity," Phys. Rev. D 64, 024024 (2001) [arXiv:hep-th/0009083].

[30] T. Gherghetta and A. Riotto, "Gravity-mediated supersymmetry breaking in the brane-world," Nucl. Phys. B 623, 97 (2002) [arXiv:hep-th/0110022].

[31] R. Rattazzi, C. A. Scrucca and A. Strumia, "Brane to brane gravity mediation of supersymmetry breaking," Nucl. Phys. B 674, 171 (2003) [arXiv:hep-th/0305184].

[32] G. A. Diamandis, B. C. Georgalas, P. Kouroumalou and A. B. Lahanas, "On the brane coupling of unified orbifolds with gauge interactions in the bulk," Phys. Lett. B 602, 112 (2004) [arXiv:hep-th/0402228].

[33] J. A. Bagger and D. V. Belyaev, "Brane-localized Goldstone fermions in bulk supergravity," Phys. Rev. D 72, 065007 (2005) [arXiv:hep-th/0406126].

[34] A. Falkowski, "On the one-loop Kaehler potential in five-dimensional brane-world supergravity," JHEP 0505, 073 (2005) [arXiv:hep-th/0502072].

[35] I. G. Moss, "Boundary terms for eleven-dimensional supergravity and M-theory," Phys. Lett. B 577, 71 (2003) [arXiv:hep-th/0308159].

[36] I. G. Moss, "Boundary terms for supergravity and heterotic M-theory," arXiv:hep-th/0403106. 
[37] J. Wess and J. Bagger, Supersymmetry and Supergravity, 2nd Edition, Princeton University Press, 1992.

[38] C. W. Misner, K. S. Thorne and J. A. Wheeler, Gravitation, San Francisco: W.H. Freeman, 1973.

[39] J. W. York, Jr.; "Kinematics and Dynamics of General Relativity" in: Sources of Gravitational Radiation, ed. L.L. Smarr, Cambridge University Press, 1979, pp. 83-126.

[40] R. M. Wald, General Relativity, Chicago: University of Chicago Press, 1984. 